\title{
Experimental investigation for characterizing and improving inlet designs in rotating detonation engines
}

Andrew T. Sisler

Follow this and additional works at: https://researchrepository.wvu.edu/etd

\section{Recommended Citation}

Sisler, Andrew T., "Experimental investigation for characterizing and improving inlet designs in rotating detonation engines" (2016). Graduate Theses, Dissertations, and Problem Reports. 6650.

https://researchrepository.wvu.edu/etd/6650

This Thesis is protected by copyright and/or related rights. It has been brought to you by the The Research Repository @ WVU with permission from the rights-holder(s). You are free to use this Thesis in any way that is permitted by the copyright and related rights legislation that applies to your use. For other uses you must obtain permission from the rights-holder(s) directly, unless additional rights are indicated by a Creative Commons license in the record and/ or on the work itself. This Thesis has been accepted for inclusion in WVU Graduate Theses, Dissertations, and Problem Reports collection by an authorized administrator of The Research Repository @ WVU. For more information, please contact researchrepository@mail.wvu.edu. 


\title{
Experimental Investigation for Characterizing and Improving Inlet
} Designs in Rotating Detonation Engines

\author{
Andrew T. Sisler \\ Thesis submitted to the \\ Benjamin M. Statler \\ College of Engineering and Mineral Resources \\ at West Virginia University \\ in partial fulfillment of the requirements for the degree of
}

\author{
Master of Science \\ in
}

Mechanical Engineering

Andrew C. Nix, Ph.D., Chair

Donald H. Ferguson, Ph.D.

Patrick H. Browning, Ph.D.

Department of Mechanical and Aerospace Engineering

Morgantown, West Virginia

August 2016

Keywords: Inlet design, pressure feedback, shockwave, detonation

Copyright (C) 2016, Andrew T. Sisler 


\title{
ABSTRACT \\ Experimental Investigation for Characterizing and Improving Inlet Designs in Rotating Detonation Engines
}

\begin{abstract}
Andrew T. Sisler
Rotating detonation engines (RDEs) present great potential for significant improvement in efficiency for land based power generation systems, in addition to aircraft propulsion devices. They offer the advantage of a net pressure gain across the combustor, as well as high exhaust temperatures and less entropy production due to detonative combustion. These improvements provide direct correlation to improved overall efficiency and thermal efficiency of gas turbine engines. RDEs surpass their conventional combustor counterparts in terms of their geometric size and simpler mechanical design. Among many areas of much needed research to further the technology readiness level (TRL) of RDEs, the inlet design is paramount to the successful operation of a rotating detonation engine. The inlet is one of the central impetuses behind current RDE research.
\end{abstract}

The existing inlet designs for RDEs in the research community are not optimized for maximum performance, yet are mostly used to operate research combustors. They are shown to induce high pressure drop, anywhere from 50-90\%, and provide insufficient mixing for the inlet reactants. They also provide poor interaction between channel pressure fluctuations and detonation propagations. For these reasons, novel inlet design concepts are devised and tested in this work. The primary goal of the work is to design an inlet that is well isolated from the combustion channel, and is conducive to short interruption times of its refueling capability due to shockwave passes. This will precede the loss reduction efforts to the inlet. A combustor from the Air Force Research Lab (AFRL) serves as the baseline geometry for all testing conducted. A linear lab scale testing device, which is a scaled model of the full size cylindrical RDE to allow for lower flow rates and pressures to be used, has been developed for more simplified and rapid experimental testing of inlet concepts. Novel inlet geometries are designed and created using additive manufacturing techniques. Initial experiments are conducted on the baseline inlet and are used as comparison experimental results of new inlet designs. Geometric characteristics are leveraged for their acoustic and resonant properties in order to provide the highest backflow prevention. Experimental results for each design are presented and evaluated. High-speed Schlieren video is used to supplement the quantitative data reported, and is used to analyze the flow structures and interactions with detonation. Novel inlet concepts are presented that show capability to reduce the pressure influence of detonation by $1-2 \%$, and improve the refueling time of the injectors.

Improvements from the baseline inlet consist of improvements in backflow length by up to $60 \%$, as well as reduction in recovery times from $20-30 \%$. 


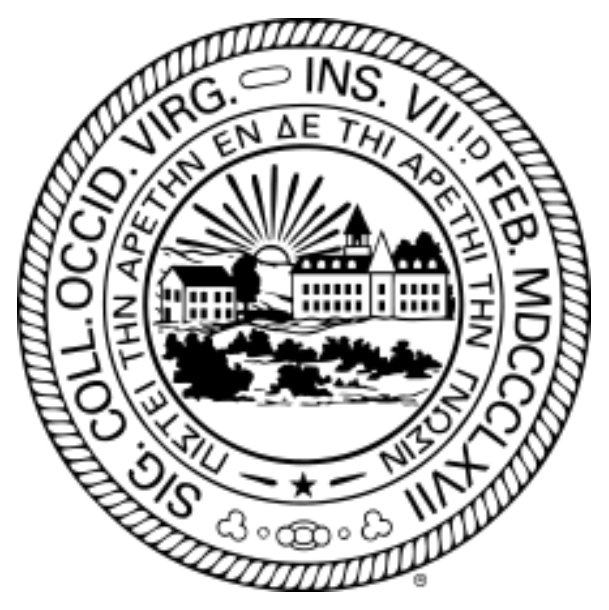

WARNING - This document contains technical data whose export is restricted by the Arms Export Control Act (Title 22, U.S.C., Sec 2751, et q.) or the Export Administration Act of 1979, as amended, Title 50, U.S.C., App. 2401 et seq. Violations of these export laws are subject to severe criminal penalties. Disseminate in accordance with provisions of DoD Directive 5230.25.

This research was supported in part by an appointment to the National Energy Technology Laboratory Research Participation Program, sponsored by the U.S. Department of Energy and administered by the Oak Ridge Institute for Science and Education. 


\section{Dedication}

I want to first dedicate this work to my wonderful wife, Krista. I had the honor of getting to transition from being her fiancé to her husband during my time in graduate school. Her support has been unending to me since the beginning of this journey. Through many apprehensions and hesitations, her affirming words of 'we were going to grad school' were the final confirmation to me that it was the right decision to attend graduate school in the first place. She has encouraged and assisted me in every way possible. Whether it would be reading through documents to make edits or playing a game of cards, she has been by my side through everything - for which I am very grateful.

I want to also acknowledge some people that have taught me countless, valuable lessons about engineering and hard work. More so than just that, they have taught me to think practically and with common sense, qualities that are impossible to teach from a textbook. I have many memories from working on the farm and in the shop with my grandfather, dad, and brother that will last a lifetime. They are some of the best carpenters, plumbers, masons, mechanics, electricians, machinists, farmers, engineers,

and hard workers that exist. The common saying goes 'jack of all trades - but master of none'. However, they have truly mastered many things. They have helped teach me pragmatic, engineering-related lessons over my lifetime. These are also skills that can never be gained from sitting in a classroom.

Throughout my time in higher education, I have seen many examples of poor leadership. Not just within the education system, but within organizations and the corporate world as well. I have been burdened by this lack of leadership ability. As a result, I desire to use my engineering knowledge, in conjunction with leadership ability to make a noticeable difference somehow in the world. I believe the Bible has many lasting principles to teach regarding leadership. More importantly, I hope that my attendance in graduate school, and my life hereafter, serves to glorify and magnify God. 
"We must be silent before we can listen. We must listen before we can learn. We must learn before we can prepare. We must prepare before we can serve. We must serve before we can lead."

\section{-William Arthur Ward}

With integrity of heart he shepherded them, with skillful hands he led them.

-Psalm 78:72 


\section{Acknowledgements}

I have many people to acknowledge and thank for their help through this work. Dr. Andrew Nix, Dr. Donald Ferguson, and Dr. Patrick Browning were helpful in guiding me through the process of thesis writing and preparation. I was blessed to be able to work around many experienced, clever people. I learned from many experienced people at NETL, all for whom I am thankful. Pete Strakey, Don Ferguson, Todd Sidwell, and Geo Richards all taught me things throughout my tenure at NETL. I am thankful as well for Doug Straub's continued mentorship and friendship. Arnab Roy and Clint Bedick were integral in teaching me the ins and outs of experimentation and lab work. I had a lot of fun working with Clint, and learning from him about Schlieren diagnostics and many other things.

Mark Tucker and Jeff Riley could not have been more helpful with performing

work in the lab. No matter what was needed, they were always able to help make it happen. The laughs and friendship we shared together were greatly appreciated. 


\section{Table of Contents}

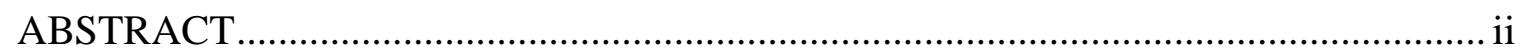

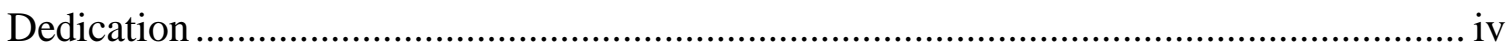

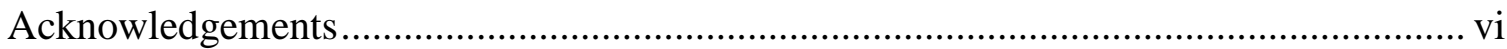

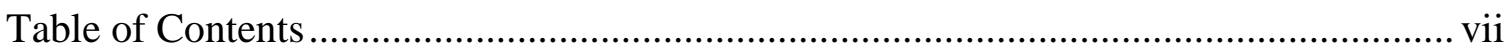

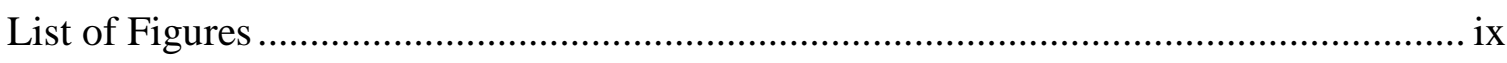

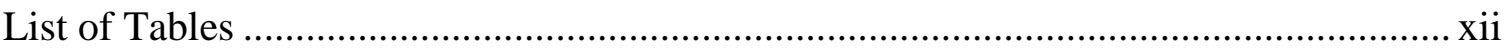

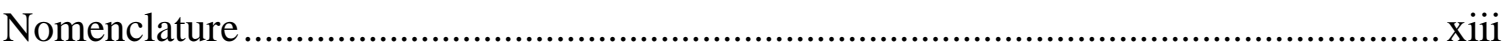

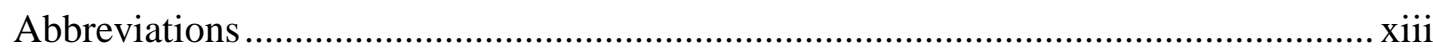

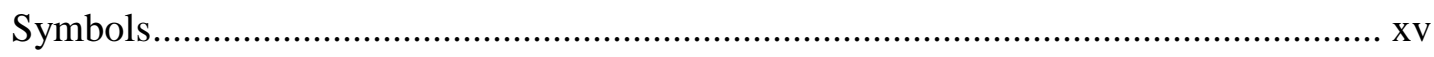

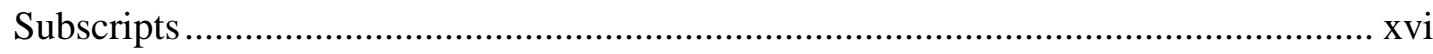

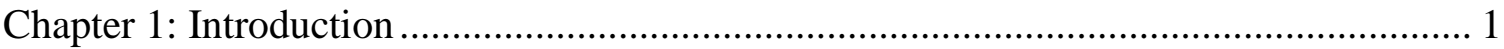

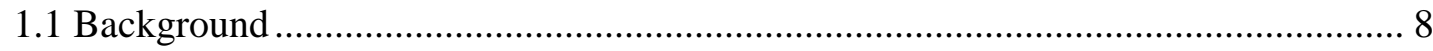

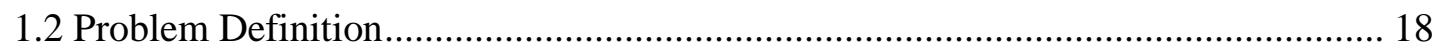

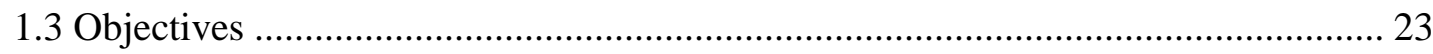

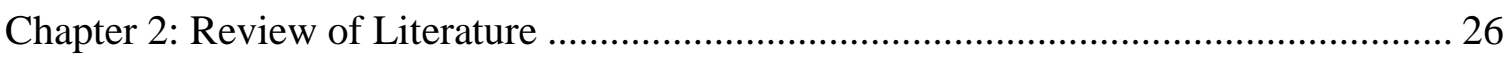

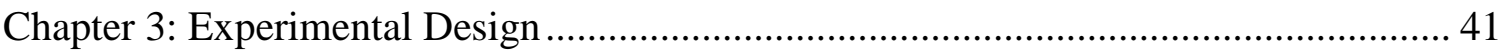

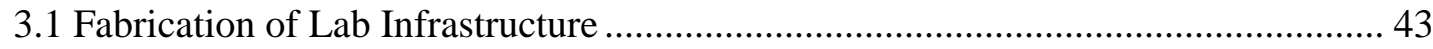

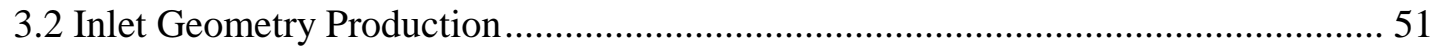

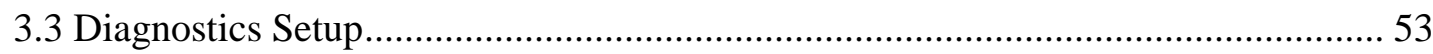

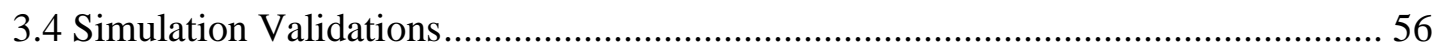

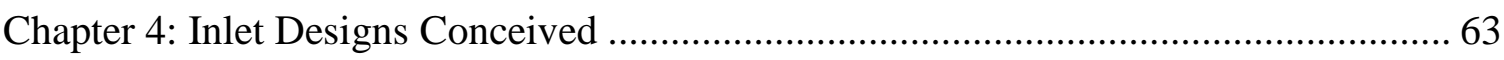

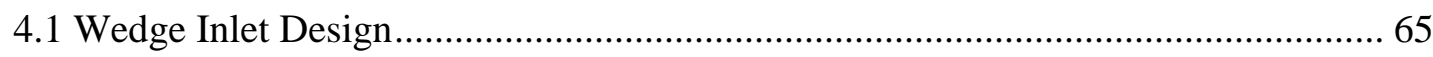

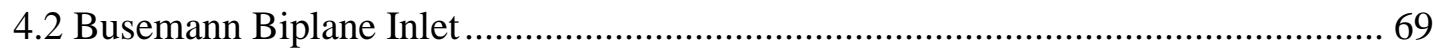

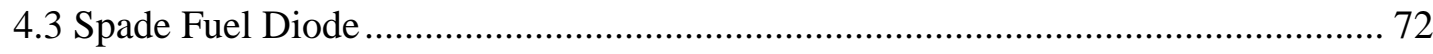

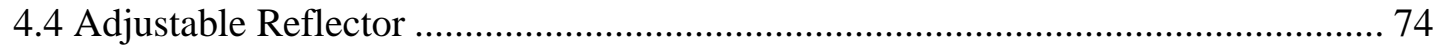

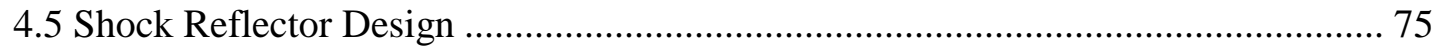

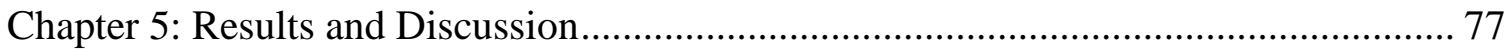

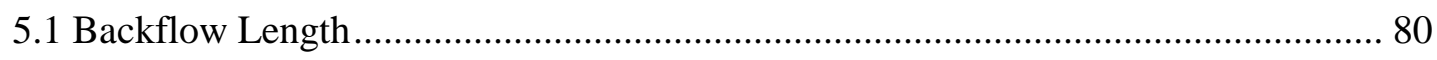




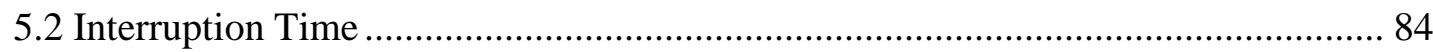

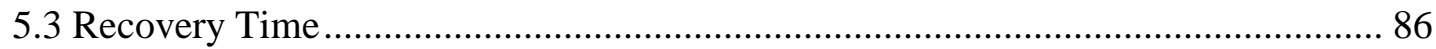

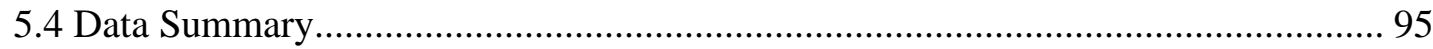

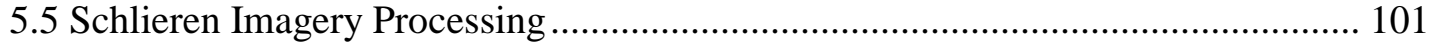

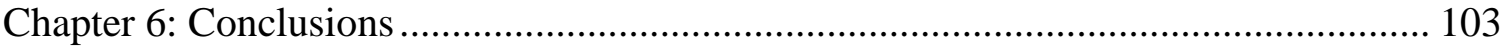

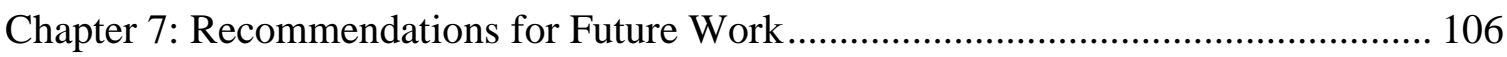

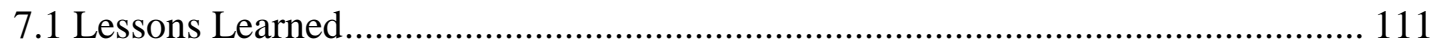

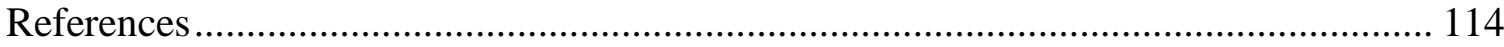

Appendix A: Data Processing \& Design Calculations MATLAB ................................ 122

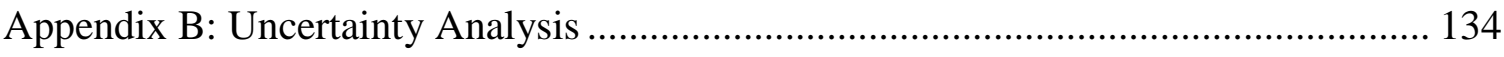




\section{List of Figures}

Figure 1. T-s and p-v diagrams comparing four major thermodynamic cycles [5] ................ 2

Figure 2. Variants of aero-engines, a) turbojet, b) ramjet, c) scramjet, [10] ........................ 6

Figure 3. Negative feedback loop analogous to RDE inlet.......................................... 7

Figure 4. PDE reed valve from Ma et al. [12]: Undeformed on left, deformed on right......... 9

Figure 5. Actively controlled rotating ball valve [13] .................................................. 10

Figure 6. Pressure drop across baseline inlet, 0.022 inch air gap inlet ............................... 11

Figure 7. RDE schematic detailing major components and regions [17] .......................... 13

Figure 8. Baseline inlet geometry with meshing done for thermal analysis ........................ 13

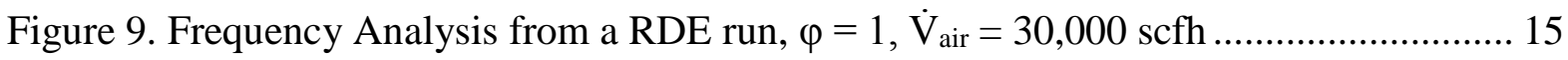

Figure 10. Inlet depictions-Top: aircraft propulsion inlet, Bottom: RDE inlet application . 16

Figure 11. Illustration of inlet backflow phenomena [19] ............................................ 19

Figure 12. Mach number contours, after 0.5 wave revolution has passed by (left), and just

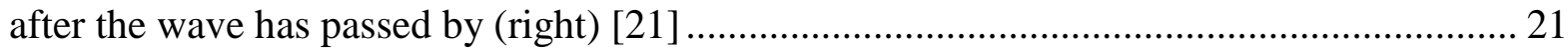

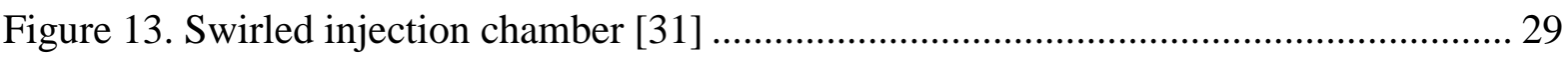

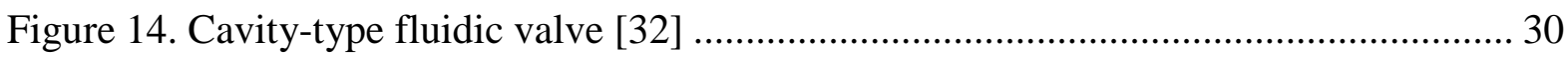

Figure 15. Density solution for cavity-slot injection design [26] ..................................... 31

Figure 16. Original inlet design from AFRL, Shank et al. [36].......................................... 33

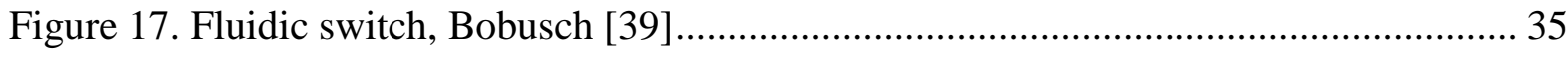

Figure 18. Temperature (K) solution for cavity slots-wave propagation is left to right [26] . 36

Figure 19. Scroll diode with 'tulip like' geometry on centerline [39] ................................. 37

Figure 20. Free expansion nozzle, first proposed by Barakauskas et al. [41] ...................... 38

Figure 21. Cylindrical combustor extruded into linear lab scale rig.................................. 41

Figure 22. Housing for inlet testing with detonation tube mounted ................................. 42

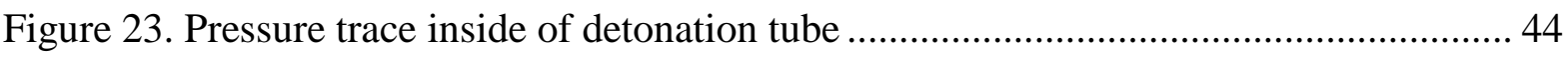

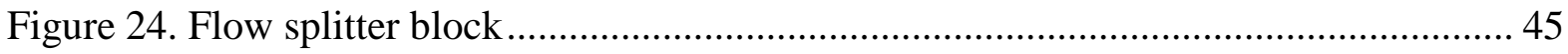

Figure 25. Pressure trace of detonation tube after splitter block ...................................... 47

Figure 26. Instrumentation locations on LSRDE, outerbody ports on left, plena on right..... 52 
Figure 27. Conventional Schlieren technique [51]

Figure 28. Optical arrangement for high-speed Schlieren diagnostics .............................. 54

Figure 29. Reflector Design 1-top left, Design 2-top right, Design 3-bottom............... 58

Figure 30. Simulation results for reflector designs for mass fraction, pressure, $x$ \& y-velocity

Figure 31. Directions of reference in the RDE

Figure 32. Hydrogen mass fraction contours at injection plane-baseline on left, wedge

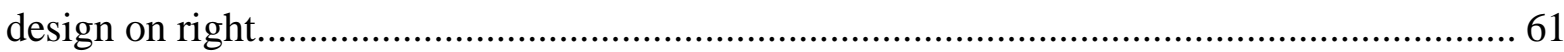

Figure 33. X-Velocity contours — baseline on left, wedge design on right ........................ 62

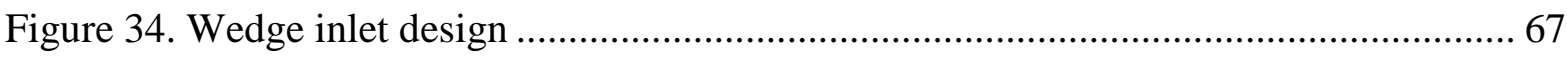

Figure 35. Portrayal of Busemann opposing wedge theory [66] ...................................... 69

Figure 36. Design rendering of Busemann inlet (throat of nozzle) ................................... 71

Figure 37. CAD view of fuel section modification made to each fuel injector .................... 72

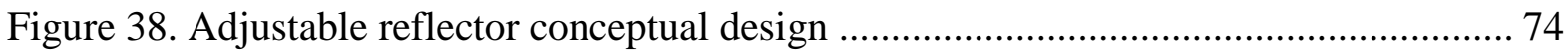

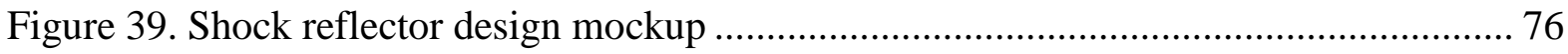

Figure 40. Two successive Schlieren frames of shock structure in inlet plenums, 0.022" gap

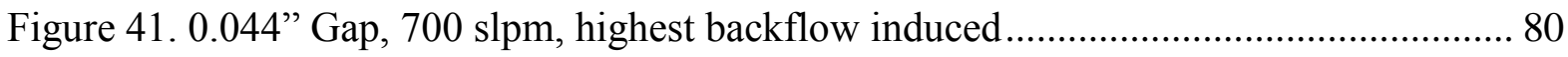

Figure 42. Wedge design, highest backflow experienced............................................. 81

Figure 43. Maximum backflow for split path design .................................................. 81

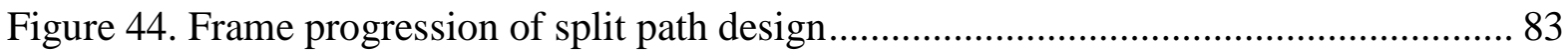

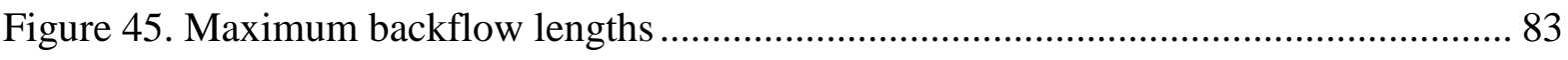

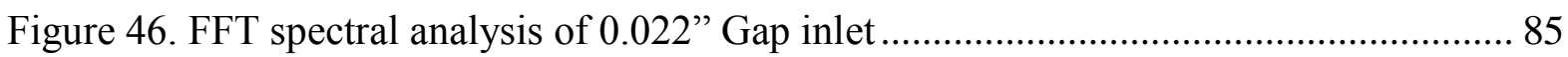

Figure 47. Data set from 0.044" Gap, 700 slpm, with interrogation windows indicated by

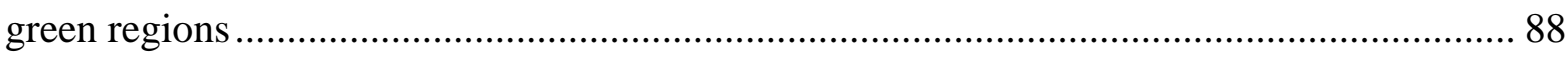

Figure 48. Maximum backflow shown for 0.022" gap (top), 0.044" gap (middle), and 0.066"

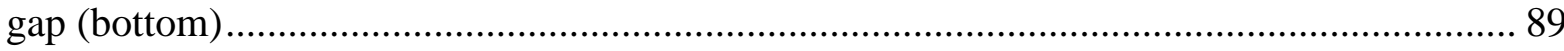

Figure 49. Evolution of shockwave through adjustable reflector, frame progressions at 0.01

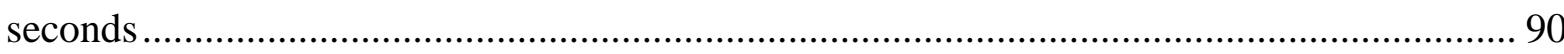


Figure 50. Busemann design at maximum backflow conditions 91

Figure 51. Wedge inlet pressure trace, $700 \mathrm{slpm}$ case ..................................................... 93

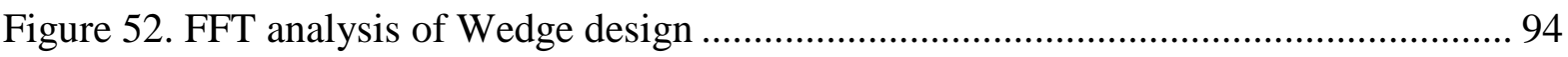

Figure 53. Variation in pressure losses of each inlet design ........................................... 96

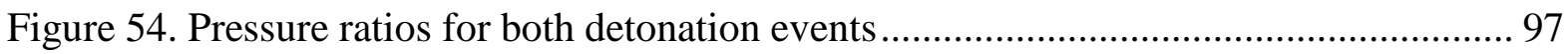

Figure 55. Pressure ratios for fuel injector designs.................................................. 98

Figure 56. Sealing design change to reduce leakage around window .............................. 112

Figure 57. Detonation byproducts imaged long after detonations extinguish .................... 113 


\section{List of Tables}

Table 1. Operating conditions for LSRDE ............................................................. 50

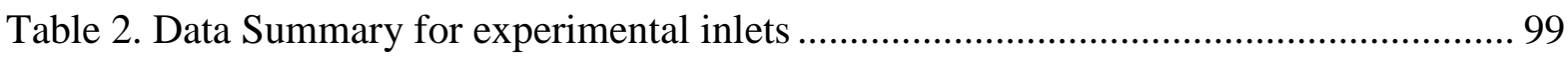




\section{Nomenclature}

\section{Abbreviations}

$\mathrm{AC}$

AFRL

CAD

CD

CFD

CJ

CVC

Da

DAQ

DBD

DDT

EHD

ED

FL

FFT

fps

FSRDE

LSRDE

MEMS
Alternating current

Air Force Research Lab

Computer-aided design

Converging-diverging (nozzle)

Computational fluid dynamics

Chapman-Jouguet

Constant volume combustion

Damköhler number

Data acquisition

Dielectric barrier discharge

Deflagration-to-detonation transition

Electrohydrodynamics

Expansion-deflection (nozzle)

Focal length

Fast Fourier Transform

Frames per second

Full scale RDE

Lab scale RDE

Microelectromechanical System 
MHD

NETL

NRL

$\mathrm{Pa}$

PDE

PGC

PLIF

ppi

PR

psig

RDC

RDE

$\operatorname{Re}$

scfh

SFC

slpm

St

$\mathrm{t} / \mathrm{c}$

TRL

UTA
Magnetohydrodynamics

National Energy Technology Laboratory

Naval Research Laboratory

Pascals

Pulse detonation engine

Pressure gain combustion

Planar laser induced fluorescence

pixels per inch

Pressure ratio

Pounds per square inch (gage)

Rotating detonation combustor

Rotating detonation engine

Reynolds number

Standard cubic feet per hour

Specific fuel consumption

Standard liters per minute

Strouhal number

Thickness-chord ratio

Technology readiness level

University of Texas at Arlington 


\section{Symbols}

A

C

D

$\varepsilon$

$\Delta$

f

$h^{*}$

$\mathrm{Hz}$

$J$

$k H z$

$\lambda$

$L$

M

mm

$\mathrm{m} / \mathrm{s}$

$\triangle P$

$P$

$q$

$S$

$t$
Area $\left(\mathrm{m}^{2}\right)$

Coefficient

Diodicity

Wedge angle (degrees)

Minimum channel width (mm)

Fuel-air mass ratio

Critical fill height (mm)

Hertz $\left(\mathrm{s}^{-1}\right)$

Momentum flux ratio

kilohertz

Detonation cell size (mm)

Backflow length (inches)

Mach number

Millimeter

Meters per second

Pressure differential (psi)

Pressure (psi)

Dynamic pressure (psi)

Specific entropy (kJ/kg-K)

Time (seconds) 


$\begin{array}{ll}u & \text { Velocity }(\mathrm{m} / \mathrm{s}) \\ \dot{V} & \text { Volumetric flow rate }(\mathrm{scfh}) \\ \varphi & \text { Equivalence ratio } \\ \eta & \text { Efficiency } \\ \dot{m} & \text { Mass flow rate }(\mathrm{kg} / \mathrm{s}) \\ \rho & \text { Density }\left(\mathrm{kg} / \mathrm{m}^{3}\right) \\ \alpha & \text { Mixing quantity } \\ \gamma & \text { Ratio of specific heats }\end{array}$

\section{Subscripts}

$\begin{array}{ll}\text { air } & \text { Air side } \\ \text { conv } & \text { Bulk flow } \\ \text { down } & \text { Convective } \\ f & \text { Downstream } \\ i & \text { Forward } \\ j e t & \text { Inlet } \\ l & \text { Jet flow } \\ \text { max } & \text { Local } \\ m & \text { Maximum } \\ p & \text { Mixing }\end{array}$




$\begin{array}{ll}r & \text { Reverse } \\ s & \text { Stoichiometric } \\ s h & \text { Shockwave } \\ t & \text { Throat } \\ \text { up } & \text { Upstream } \\ \infty & \text { Freestream }\end{array}$




\section{Chapter 1: Introduction}

$\mathrm{R}$

otating detonation combustion is rapidly gaining increased attention due to the many benefits it offers in terms of thermodynamic efficiency. Among the biggest attraction to rotating detonation engines (RDEs) is the pressure gain that develops upon detonation establishment. While most conventional combustion systems undergo a subsonic deflagration combustion process, a RDE experiences a deflagration-todetonation transition (DDT) at which it changes to the detonation regime of combustion. Deflagration systems, defined in the Brayton cycle, experience a pressure loss upon combustion from viscous dissipation, flow distortion, flame holding, mixing losses, and other mechanisms. This pressure loss is typically anywhere from 2 to 8 percent of the static pressure [1]. However, detonation creates a pressure gain, and is what holds potential for increasing engine thermal efficiency by up to $7 \%$ from Brayton cycle engines with comparable pressure ratios [2]. This is significant, considering the fact that technologies that boost turbine efficiency by tenths of a percentage are highly sought after as it is. To place it in perspective, for an engine whose annual fuel costs are $\$ 100$ million dollars, an efficiency increase of even just $0.1 \%$ would result in an annual fuel savings of $\$ 100,000$ dollars [3]. To increase that efficiency gain to $1 \%$ would boost the annual savings in fuel to an impressive $\$ 1$ million dollars. However, inherent in the pressure gain is an adverse pressure gradient that develops at the injection plane of the RDE as the shockwave passes, where the pressure from detonation exceeds the feed pressure. The pressure gradient produces a phenomenon that is detrimental to its operation. Combustion in an RDE occurs in the combustion annulus, which is referred to in this work synonymously with the combustion channel. As the detonation wave front cycles azimuthally around the combustion annulus, it tends to backflow through the inlet, being driven by the adverse pressure gradient. The pressure fluctuations experienced at the inlet to the RDE are significant. In fact, it was found that the local mean static pressure fluctuations at the inlet could reach almost 10\% higher than the pressure fluctuations at the exhaust [4]. This indicates that the inlet can experience higher pressure pulsations in an RDE than the exhaust does. This event causes a discontinuity to the filling effect for as long as the backflow of combustion 


\section{Introduction}

products overcomes the fresh, forward inlet flow. In addition to that, it has the possibility of catastrophic outcomes, such as flashback and explosions caused by ignition in unwanted upstream locations.

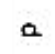

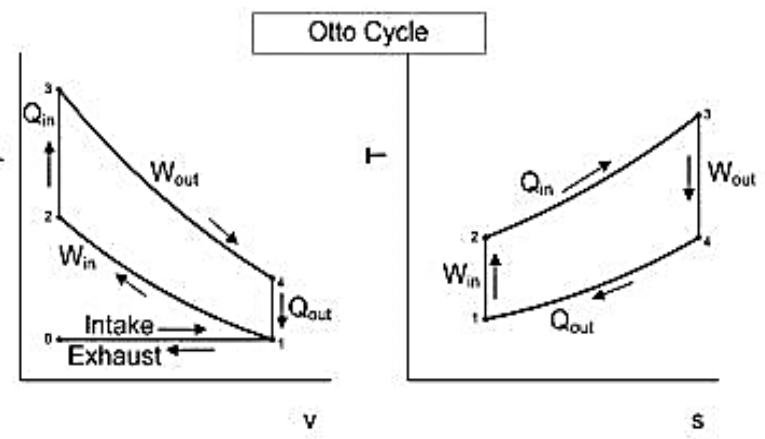

a

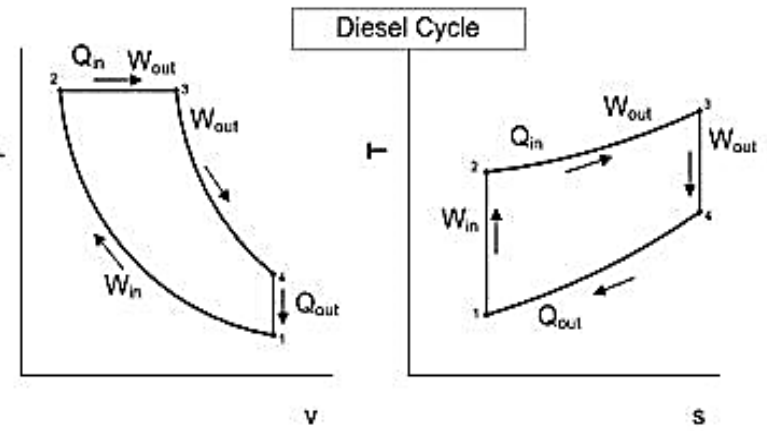

-

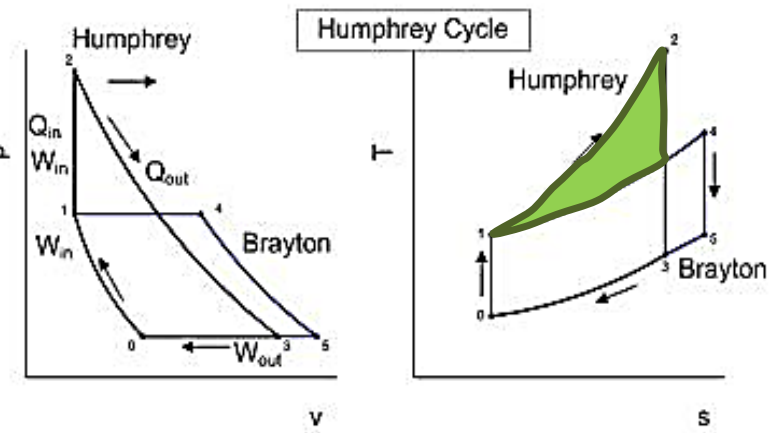

$\circ$

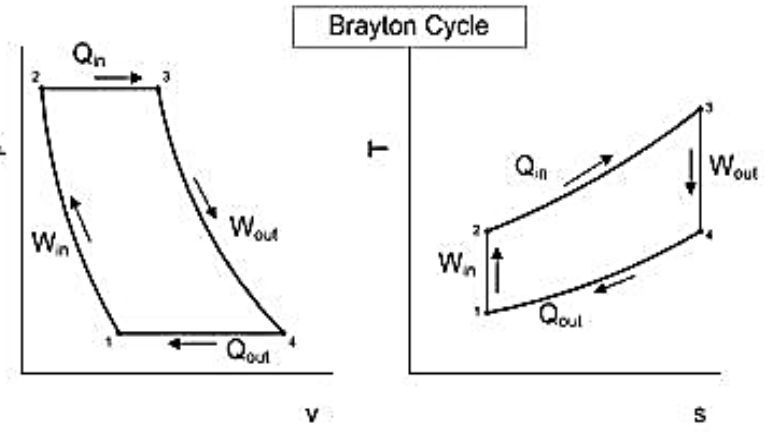

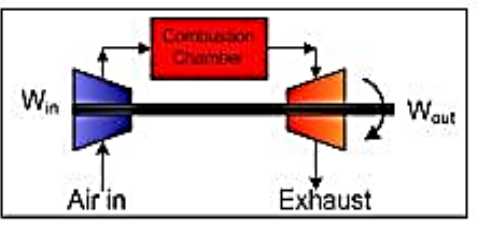

Figure 1. T-s and p-v diagrams comparing four major thermodynamic cycles [5]

The Humphrey cycle has been used to explain the thermodynamic cycle for a detonation engine, which accounts for constant volume combustion (CVC). It is a modification to the Brayton cycle, where constant volume heat addition replaces a constant pressure heat addition, shown in Figure 1. The region highlighted in green from Figure 1 represents the area that $\mathrm{CVC}$ adds to the cycle. When compared to the constant-pressure Brayton cycle, which defines the cycle of a gas turbine, the Humphrey cycle outperforms the Brayton cycle. The direct comparison of the Humphrey and Brayton cycles is made in the 


\section{Introduction}

upper right graphs of Figure 1. By estimating its cycle as a constant-volume process, the RDE generates less entropy than does a conventional gas turbine undergoing a Brayton cycle. It may not be realized through these diagrams, but RDEs can also operate at a higher thermal efficiency than conventional combustors do. The increased potential for work is greater with a RDE from several sources: its higher thermal efficiency, greater pressure availability, and lower entropy generation. In addition, the Humphrey cycle's ability to generate higher specific impulse makes the RDE desirable for propulsion applications. The higher potential to perform work makes the Humphrey cycle especially advantageous for power generation cycles. With environmentally conscience emissions restrictions becoming stricter, initiatives for more sustainable practices in energy production are increasing. An example is to improve cycle efficiency for lower fuel consumption. Being more mindful of principles such as this drives research towards pressure gain combustion devices (PGC) like RDEs.

Lower fuel consumption, higher efficiencies, mechanical simplicity, and greater power density are all additional benefits that RDE's have to offer. Although RDE's offer a long list of benefits to the science and engineering community, there are many obstacles that stand in the way of further developing and advancing the technology to a matured level. Wave bifurcation, mode switching, emissions reduction, thermal management, and unsteady exhaust flow are all areas of intensive research. Detonation combustion is much more sensitive to instabilities than deflagration combustion. If the conditions are not carefully designed and controlled, detonation is difficult to establish and sustain. These are some of the areas with the least understanding. Better characterizing and understanding these topics can lead to huge breakthroughs in this field. Perhaps the biggest impediment with the technology is the inlet design. Without a robust inlet design, regardless of how the rest of the system performs, the pressure gain system may be as good as useless. It is at the inlet where the combustion process begins.

Addressing the inlet design issue is the first crucial step in improving RDEs overall. It is helpful in understanding the importance of the combustor inlet by drawing an analogy between that of inlets for other applications. Much like in military aircraft applications, the 


\section{Introduction}

engine's inlet is often the most challenging design piece of the entire engine design project. As Ben Rich said in his book regarding the SR-71 Blackbird, the "crown of thorns was designing and building the powerful engine's inlets - the key to the engine's thrust and its ability to reach blistering speeds. This became the single most complex and vexing engineering problem of the entire project" [6]. While inlets typically perform no work on the fluid, at cruising conditions, the inlet of the SR-71 accounts for over $50 \%$ of the engine's thrust. This is quite impressive considering this is even prior to the stage where air enters through the combustion and turbine stages. However, this inlet, like other supersonic air vehicle inlets, has the benefit of being able to retract the aerospike in real-time and achieve variable geometries. This is not possible with the RDE setup. Based on this context, the criticality of a robust inlet design is clear.

RDE technology is beneficial in the sense that it is scalable. This is the reason that RDEs create interest to a wide range of companies and research groups. The same principles that make it a cutting-edge technology for aircraft propulsion can be scaled for use as a marine propulsion device. In fact, the U.S. Navy has strong interest in RDE's for marine propulsion applications and electricity generation on ships. Scalability is also the reason it is so viable for consideration in land-based power generation. Since gas turbine technology for

land-based use is not as limited by size as aerospace, RDEs provide a lot of versatility in their application to gas turbine combustion processes. RDEs are not limited to their potential for land based use. The highly impulsive nature of the exhaust flow is favorable for many propulsion applications, including rocket engines, boosters for supersonic aircraft, or for propulsion of weapons systems [7].

While maturing RDE technology as a stand-alone system is difficult, successful integration to turbine engines for RDEs create innumerable challenges. Hard start-ups, exhaust transition to the turbine, turbine blade cooling, emission levels, and thermal management are just a few on the list of engineering hurdles. There are many questions that still remain on a systems level as to the most effective means for integrating with the turbine. For instance, it may be feasible to remove several stages from the compressor, of which will 


\section{Introduction}

be compensated for by the pressure gain achieved with a RDE. In fact, if a 5\% gain in pressure is achieved, it could have in essence the same effect as doubling the compression ratio [8]. This will ensure the pressure ratio is maintained, while also generating cost and weight savings to the engine. Conditioning of the exhaust flow poses potential for a viable means of preparing the combustor's exhaust flow for entrance to the turbine. Wellconditioned exhaust flow will ensure durability and longevity of the turbine stages. The high

pressure, quasi-unsteady exhaust flow infested with shockwaves will cause many structural and design challenges for turbine engineers. The frequency of the exhaust flow will severely impact blade flutter, vibrational modes, and overall blade durability. Some work has been underway for studying the integration of a detonative combustor with a turbine engine, like that of Swamy et al. [9]. The computational analysis done comparing a detonation-based turbofan cycle to a conventional turbofan cycle demonstrates the potential for performance increase. Questions are beginning to get addressed, such as whether the detonation combustor should be in a can or annular type arrangement. Integration into some engine types may be easier, especially for engines of a scramjet type, shown in Figure 2. For this type of application, a combined cycle engine has potential, in which a scramjet and RDE are combined and result in a shorter combustor length. This type of implementation often conceives the combined cycle or hybrid engine would operate in an ejection mode. While this has not yet been demonstrated for flight applications, it has been proven preliminarily in laboratory testing. 
(a)
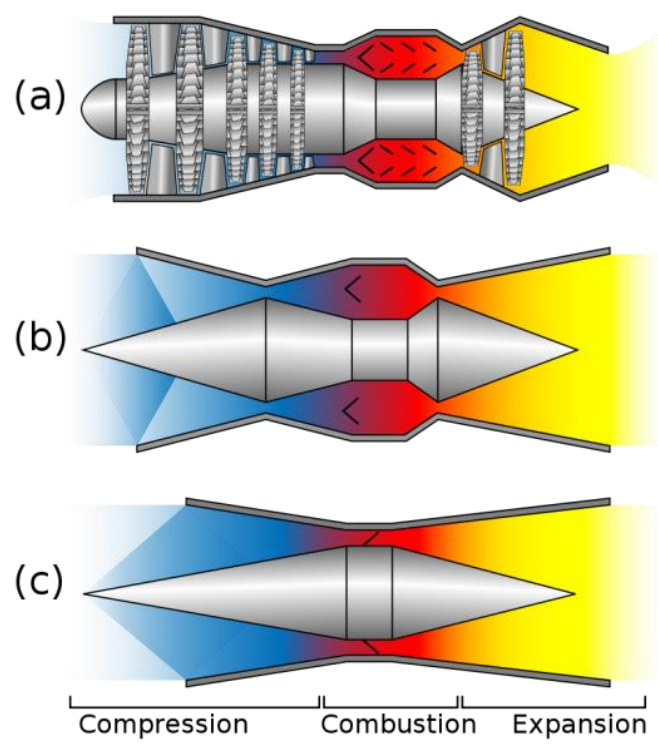

Figure 2. Variants of aero-engines, a) turbojet, b) ramjet, c) scramjet, [10]

The inlet dictates how well the reactants mix, how low the induced pressure drop is, and how well it contains the detonation wave and its products in the annulus region. A negative feedback loop is established between the inlet and the combustor. The feedback loop is the portion of the detonation wave that travels back through the inlet, causing instabilities and forward flow disturbances. For conceptualizing how an inlet works, a notional negative feedback loop is shown in Figure 3. The goal is to minimize the effective gain in the feedback loop. Ideally, the feedback loop could be entirely broken, so that the inlet can detect nothing that is occurring in the detonation channel. This will eliminate the impact that the detonation has on the inlet feed flow. 


\section{Introduction}

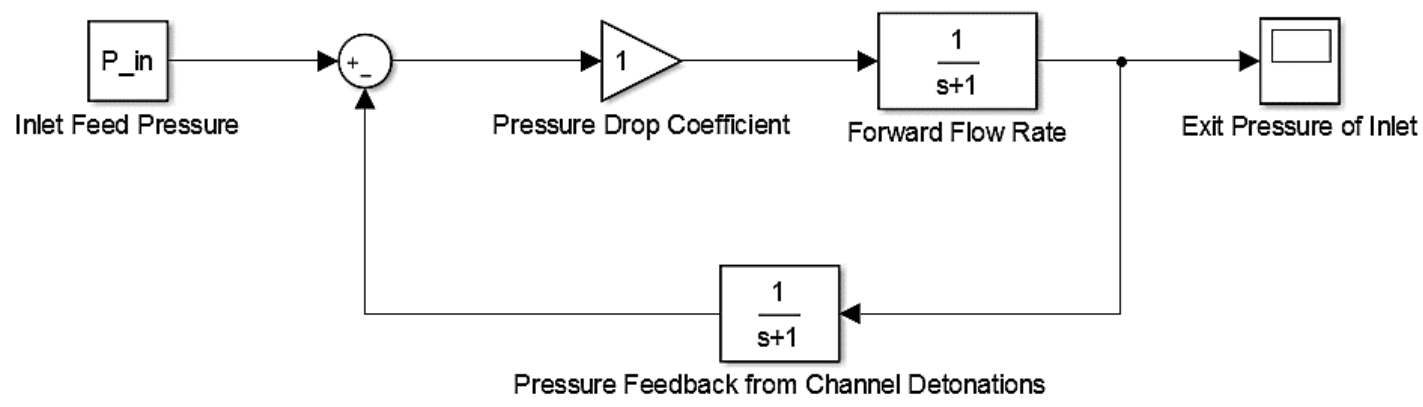

Figure 3. Negative feedback loop analogous to RDE inlet

Many studies have been performed for the sake of measuring plenum pressures, thrust, and impulse of a RDE. However, none of these studies have provided visual access to the inlet plenums. This unique capability is presented in the remainder of this work. The visual access allows the flow structures to be evaluated at a more detailed level and analyze how the combustor is interacting with the inlet geometry. Rather than simply relying on quantitative measurements alone, this enables measured values, like pressure, thrust, and heat flux, to be paired with qualitative data that is acquired. Naples et al. [11] characterized the detonation flow field using a transparent outerbody and chemiluminescence. Through this method, the regions and layers that comprise the combustion annulus were separated and identified. This work was very important for measuring oblique shock angles, and how the shear layer, deflagration boundary, and detonation waves interact with one another.

However, the chemiluminescence was not able to be applied within the inlet plenums. The flow field and shockwave dynamic effects are analyzed in this work, supported by visual data from novel inlet design cross sections. 


\subsection{Background}

A rotating detonation engine is a type of continuous detonation engine, which stems from technology termed as pulse detonation engines (PDE). Detonation is initiated only one time in RDEs by an initiator tube that enters tangentially with the annulus. The detonation wave travels circumferentially around the annulus, and is self-sustaining barring any flame outs. It has many advantages to the PDE, as it is self-sustaining, does not require multiple ignitions, and operates on a thermodynamic cycle that offers more efficiency benefits. However, the operating frequency of the RDE is on the order of $\mathrm{kHz}(1-10 \mathrm{kHz})$, while that of the PDE is on the order of $\mathrm{Hz}(100-500 \mathrm{~Hz})$, which makes the exhaust flow of RDEs steadier than PDEs [12]. This high-frequency causes many difficulties in flow control, fuel and air injection, and experimental measurement techniques.

At the advent of PDE research, controlling, designing, and managing inlet flow proved to be perplexing, and as a result, many strategies have been examined for the inlet solutions of a PDE. The details of designing a robust inlet design for RDEs will be discussed in more detail in the following sections. For RDEs, the phenomena of backflow, which is when the detonation and its associated products travel upstream into the inlet, is one of the primary obstacles the RDE inlet faces. One common approach to prevent backflow effects in a PDE are lobe shaped reed valves, as shown in Figure 4. However, reliability is a downfall of these valves. After a short duration of run time, these valves wear from high cycle fatigue and thermal stressing. As they wear, their material properties change, which changes their harmonic response to the detonation. The fatigue and degradation of the valve is clear from the used valve on the right. 

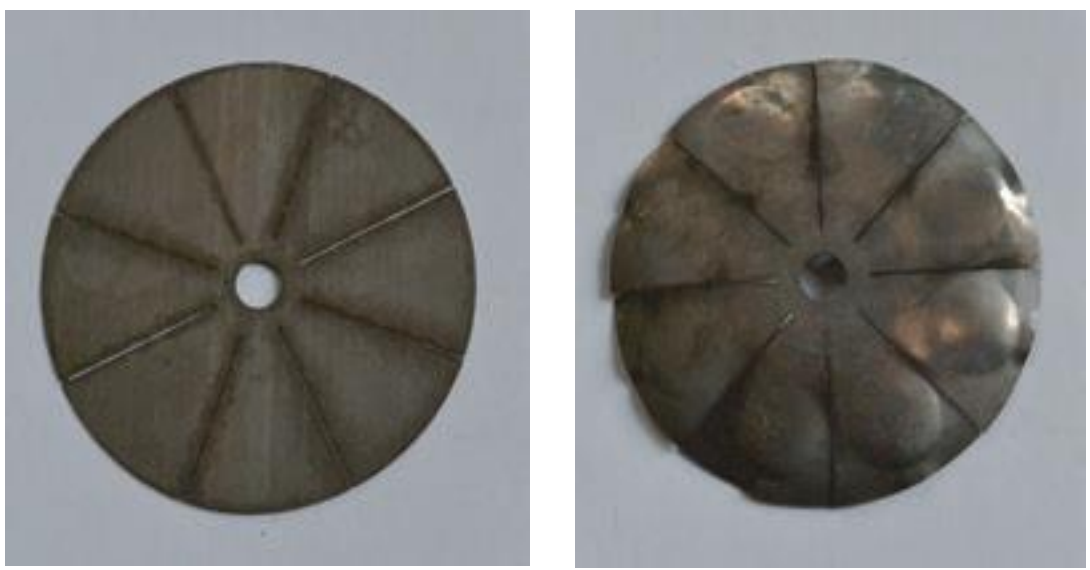

Figure 4. PDE reed valve from Ma et al. [12]: Undeformed on left, deformed on right

This issue prevents the valves from responding in the way that they were designed, and consequently, they are only viable for very short run times, which does not make it a pragmatic injection solution. Other actively controlled valves, like the rotating ball valve seen in Figure 5, have been used for PDE applications. While these prove to be more reliable than reed valves, they are challenging to control accurately enough to gain the most throughput and energy during the fill-fire-purge sequence. Therefore, it is deduced that valves such as this would not be capable of functioning properly inside of a RDE either. The mechanical properties of the material would limit the response time of each valve, preventing them from responding at a fast enough rate for detonation cycles in a RDE. The lack of robustness in valving mechanisms such as this is a major reason that PDE technology was overlooked for the sake of ventures into RDEs. 


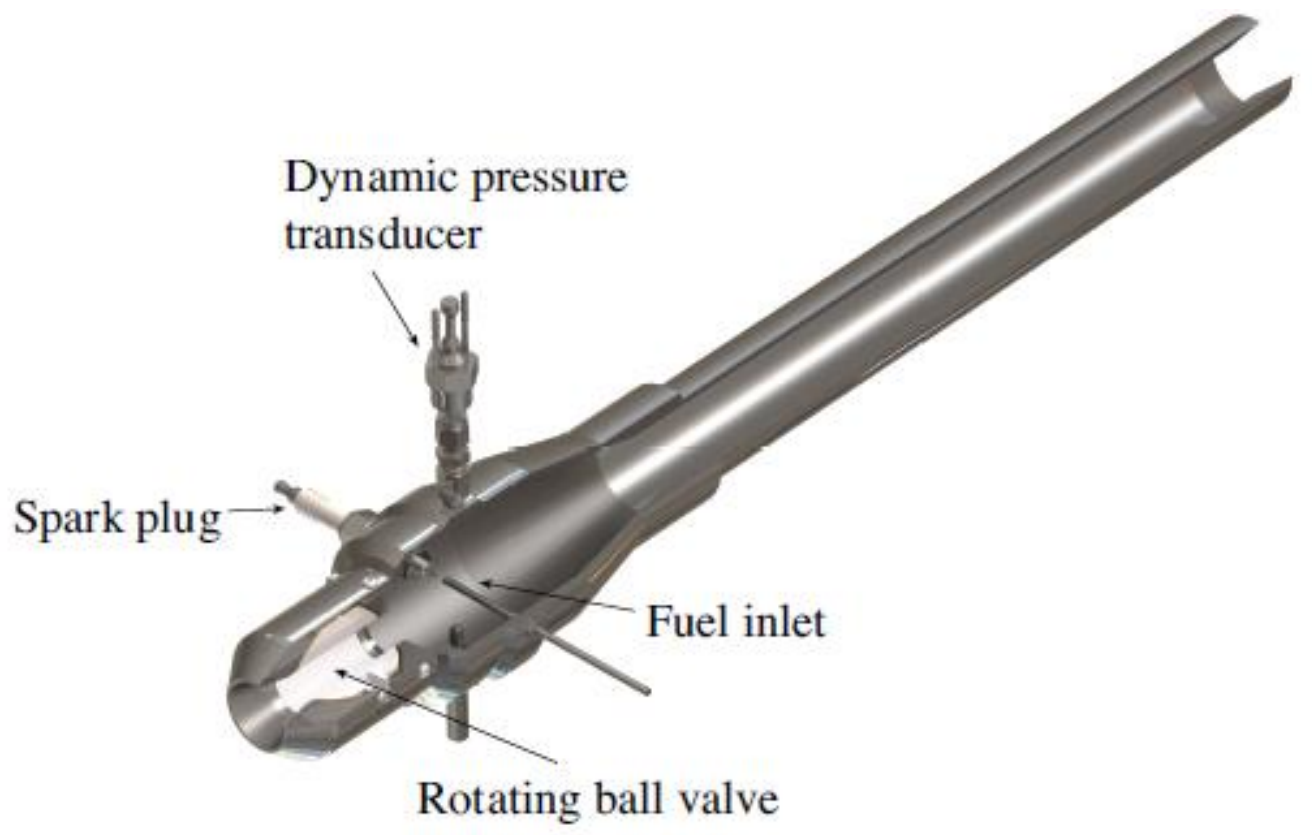

Figure 5. Actively controlled rotating ball valve [13]

Because of the frequency order for a PDE, electronically controlled fuel injectors have been implemented for use in PDE inlets [14]. Similar to automotive style injectors, they must have fast enough response to adequately provide the system with the fuel/oxidizer flow rates required. However, it is difficult to find an injector or microelectromechanical system (MEMS) that is able to reliably function in the $\mathrm{kHz}$ response range. Even for the notably lower operating frequencies of PDEs, it is difficult to control mixture injection with a highfrequency electronic valve or a rotary valve assembly [15][16]. 


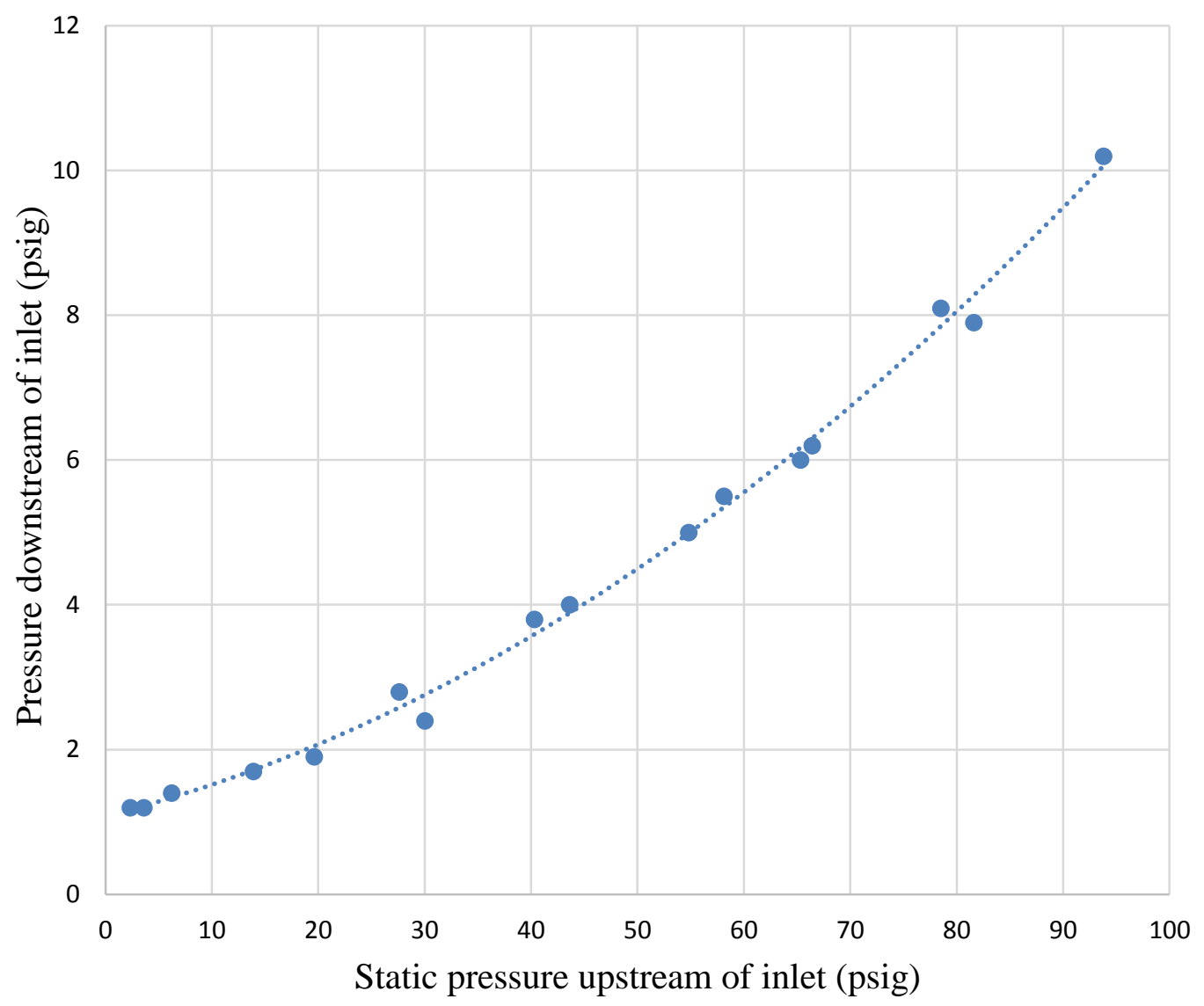

Figure 6. Pressure drop across baseline inlet, 0.022 inch air gap inlet

Thus far in RDE research, inlet design strategies have been targeted towards passive, flow geometry inlets. Figure 6 shows how the downstream pressure of the inlet changes with the upstream pressure, and exemplifies the significant pressure drop. The current inlet design used as a baseline, referenced in Figure 8, was designed at the Air Force Research Lab (AFRL). It is chosen as the baseline, because it is the design that has been in use in the majority of recent RDE research. This design poses a multitude of issues as a combustor inlet, primarily due to its large pressure drop induced on the inlet flow. This trend gets accentuated as feed pressure increases. On a system in which a pressure gain is targeted, it is 
imperative to minimize any effects that will cause pressure loss. The baseline inlet is similar in design to a converging-diverging (CD) nozzle. It chokes the flow so that the exit pressure of the inlet $\left(P_{\text {down }}\right)$, is less than the upstream pressure of the inlet $\left(P_{u p}\right)$. This also allows direct control over the mass flow injected. In theory, due to the pressure gradient this should prevent common subsonic flow from traveling backwards through the inlet. However, when a detonation wave passes by a certain point, the local pressure from the shock front exceeds the upstream inlet pressure. The pressure gradient then reverses, where $P_{\text {down }}$ is greater than $P_{u p}$, which is the driving force for pressure feedback into the inlet.

By running cold air flow through the RDE without any combustion, a distribution of the pressure measured immediately upstream of the inlet versus pressure immediately downstream of the inlet is determined. From the near-quadratic plot in Figure 6, it can be realized that there is approximately a $90 \%$ pressure drop across the baseline inlet. It is important to note that the air gap of this inlet can be modified by shimming the center body. Inlets of different air gap sizes are addressed in Chapter 4. Shims can be placed under the centerbody, represented in Figure 8 by the red shapes. The pressure profile from Figure 6 was measured at a standard air gap with no shims in place. All of the hot fired RDE tests at NETL up to this point have been run with this same air gap as well. It can be seen that on the lower end of the upstream pressure, there is less loss in pressure. However, as the upstream pressure reaches $30 \mathrm{psig}$, the trend becomes more linear. This indicates that the inlet is not well suited for lower supply volumetric flow rates or pressures. Figure 7 shows a detailed cross section of the RDE, with labeled components. The blue boxed region indicates the inlet plena, which is further expounded in Figure 8. 


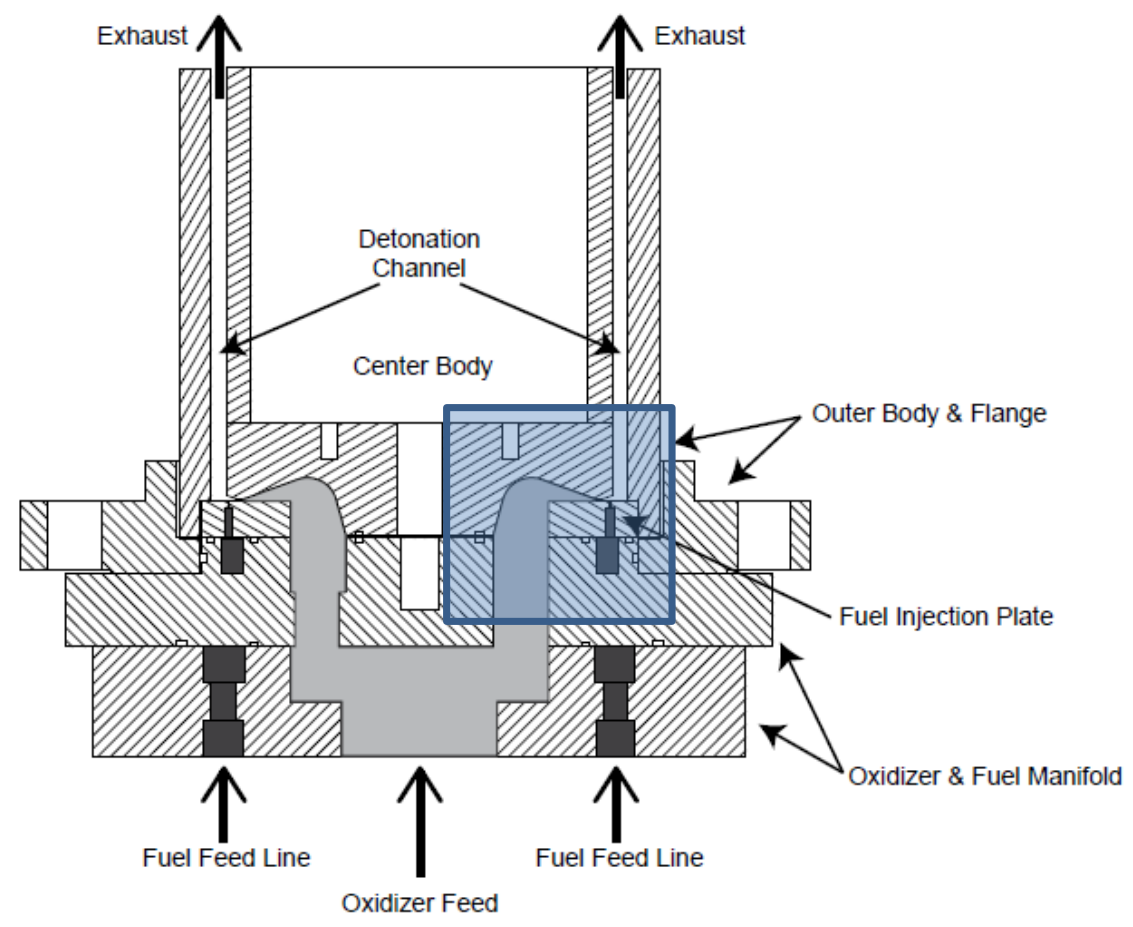

Figure 7. RDE schematic detailing major components and regions [17]

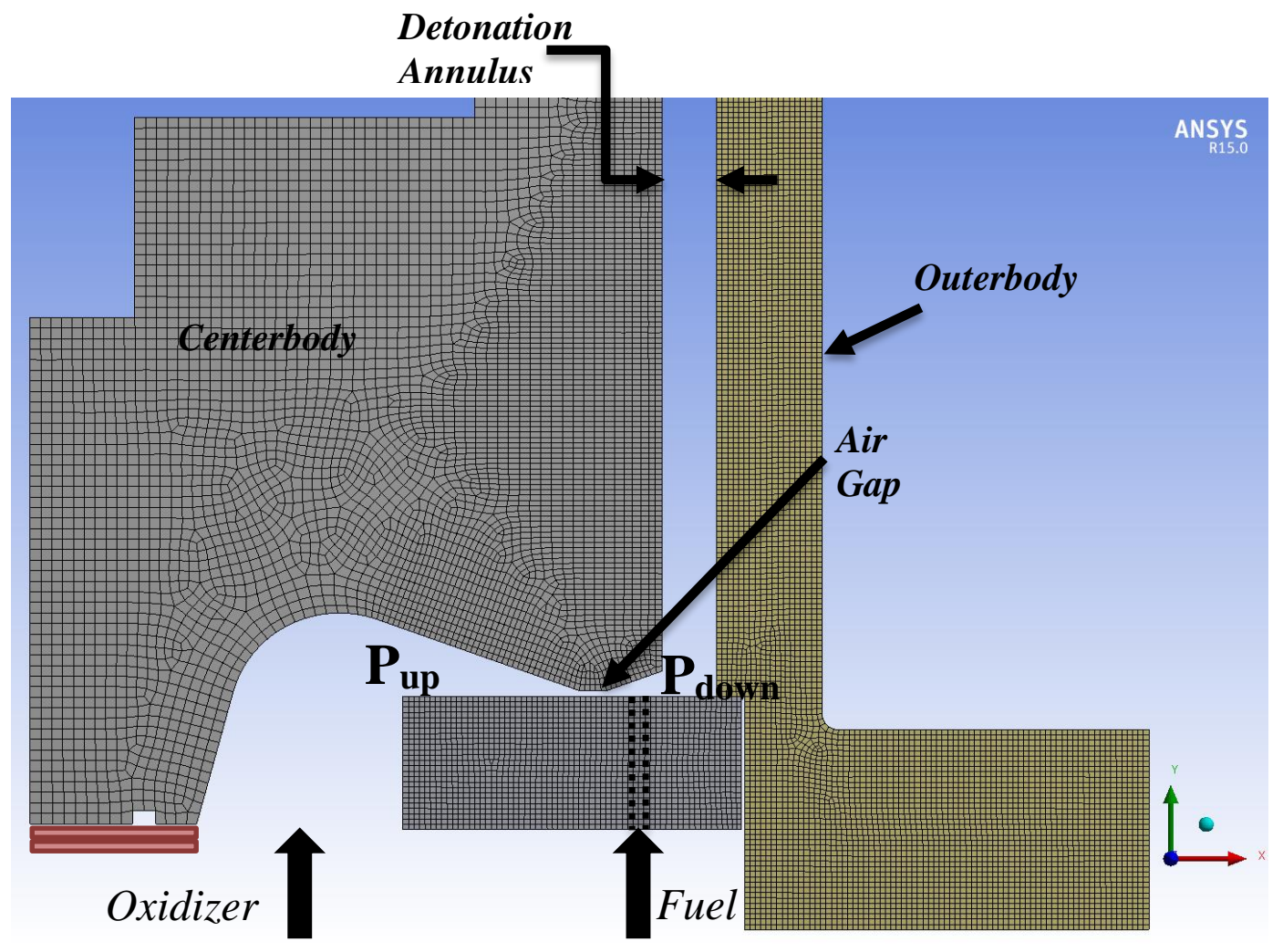

Figure 8. Baseline inlet geometry with meshing done for thermal analysis 
Understanding how the inlet operates in communication with the main combustion channel is crucial for targeting an optimal novel inlet design. The inlet design dictates how well the filling process occurs. The design shown in Figure 8 is a non-premixed RDE, which introduces separate air and fuel streams to produce the combustible mixture that fills the annulus and is then detonated. By measuring pressure history in the channel and air inlet plenum, correlations between the two sets of data provides a description of how they relate. For instance, for the case of designing acoustic filters or resonators, the frequency is an essential design parameter. While the frequency of the detonation wave can be accurately measured within the detonation channel, it must be well measured in the inlet plenum also. Figure 9 shows a detailed frequency analysis from a five second test in the hot fired RDE. The four spectra shown are derived from pressure transducer data. The first plot is that acquired in the fuel inlet plenum, two are from transducers at various circumferential locations in the RDEs outerbody, and the final plot is from the air inlet plenum. The RDE operation for this given condition seems to have two fundamental frequencies, one around $3.6 \mathrm{kHz}$, and the other around $6.5 \mathrm{kHz}$. This most likely corresponds to a wave switching mode, in which the RDE started out in a one-wave mode, cycling at just under $4 \mathrm{kHz}$. When it bifurcated into two waves, the frequency increased to $6.5 \mathrm{kHz}$. The effective wave speed decreased slightly due to the presence of two waves. The two-wave mode is clearly the dominant operation mode. The important observation to make is about the pressure response in the inlet plenums. While it has less magnitude than within the detonation channel, the fundamental frequencies directly relate to the frequency of the detonation passing. The air plenum receives around $20 \%$ of the pressure magnitude that is measured in the detonation channel. The fuel plenum responds differently to the detonation passing, since it has different flow rates, pressures, and a different geometry causing different frequencies to be excited. This has also been confirmed in other research. 

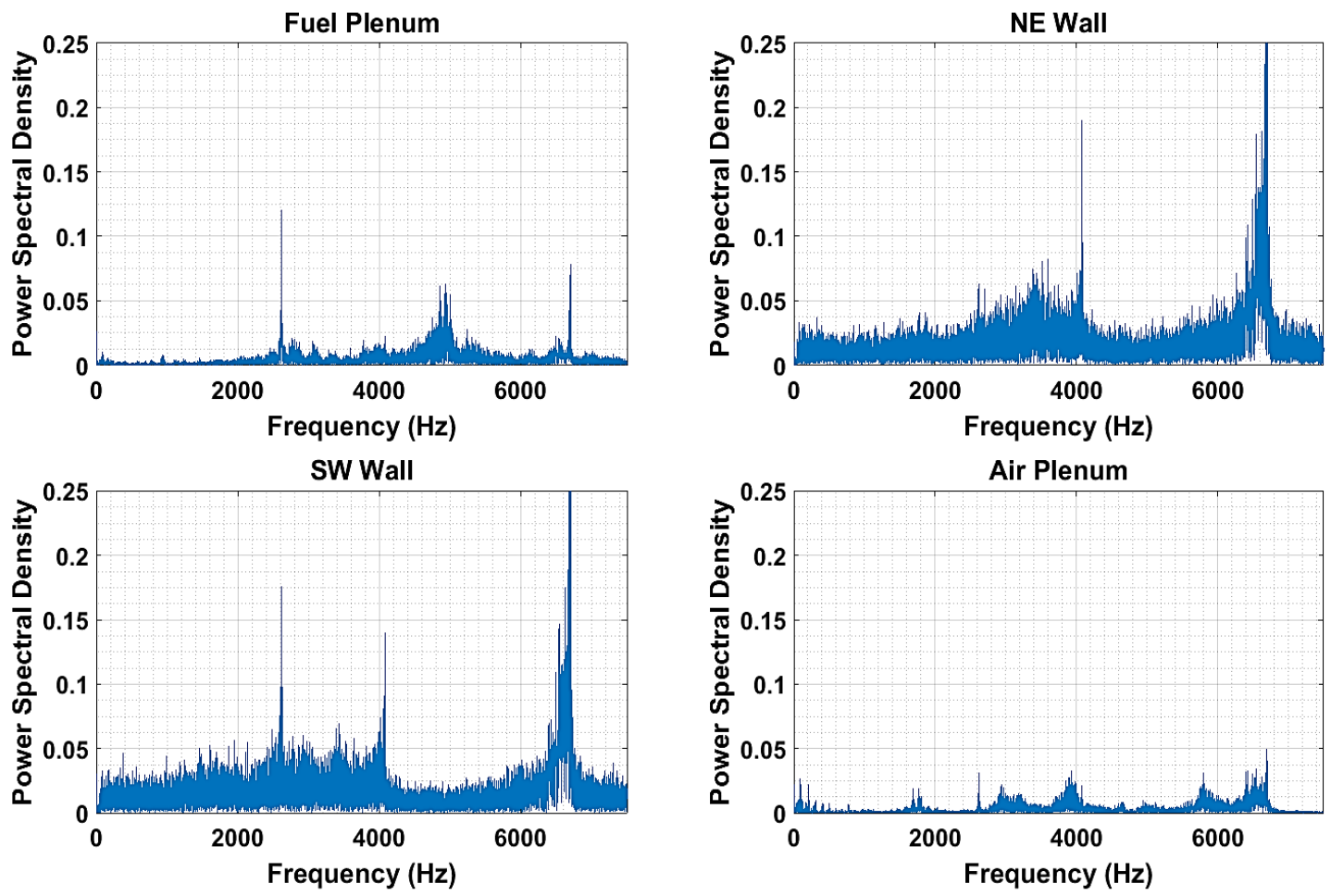

Figure 9. Frequency Analysis from a RDE run, $\varphi=1, \dot{V}_{\text {air }}=30,000 \mathrm{scfh}$

Not only does the high amount of choking lead to pressure loss, but the sharp turning of the flow contributes to the pressure drop. The flow enters fully axial, and is immediately turned 90 degrees towards the outwards radial direction. From basic fluid mechanics, these are two major sources of a pressure loss for any system.

While the design intent of a RDE inlet is vastly different from a supersonic propulsion inlet, it is helpful to make comparisons between the two for design purposes. There are many striking similarities between the two. The design intent of a RDE inlet can almost be thought of as a reversed propulsion inlet. For supersonic propulsion inlets, the main assignment for the inlet is to slow the inlet flow down as quickly as possible and eliminate the shockwaves before traveling too far through the engine system. To prevent damage to the internal components of the engine, the flow must be slowed down rapidly before passing from the inlet to the remaining portion of the engine. For hypersonic flight vehicles, this may be a speed reduction of almost $85 \%$. It must also allow for the appropriate 
mass flow of air through the inlet to allow for normal engine operation. If this is not done properly, the inlet faces difficulties such as unstart and instability, which will cause erratic operation of the aircraft. An inlet unstart is when the mass flow rate of the exhaust is less than the mass flow at the inlet. This essentially means that the inlet flow and shock structures are not properly "swallowed" by the inlet, which causes spillage around the cowl lip.

However, for the RDE, the inlet flow to the engine is at subsonic speeds. Rather than slowing down flow from the inlet to exhaust, its flow experiences a speed increase from intake to exhaust. Therefore, it must prevent the supersonic flow from traveling upstream in the undesired direction. Although there are similarities between the RDE and propulsion engine inlets, there are some differences which are imperative to identify. For an engine inlet, the freestream supersonic Mach number is caused by the forward motion of the flight vehicle, whereas it is caused by combustion in an RDE. Also, the flow at an engine inlet can often be considered adiabatic, while that approximation is not nearly as accurate for an RDE. This distinction is illustrated in Figure 10.
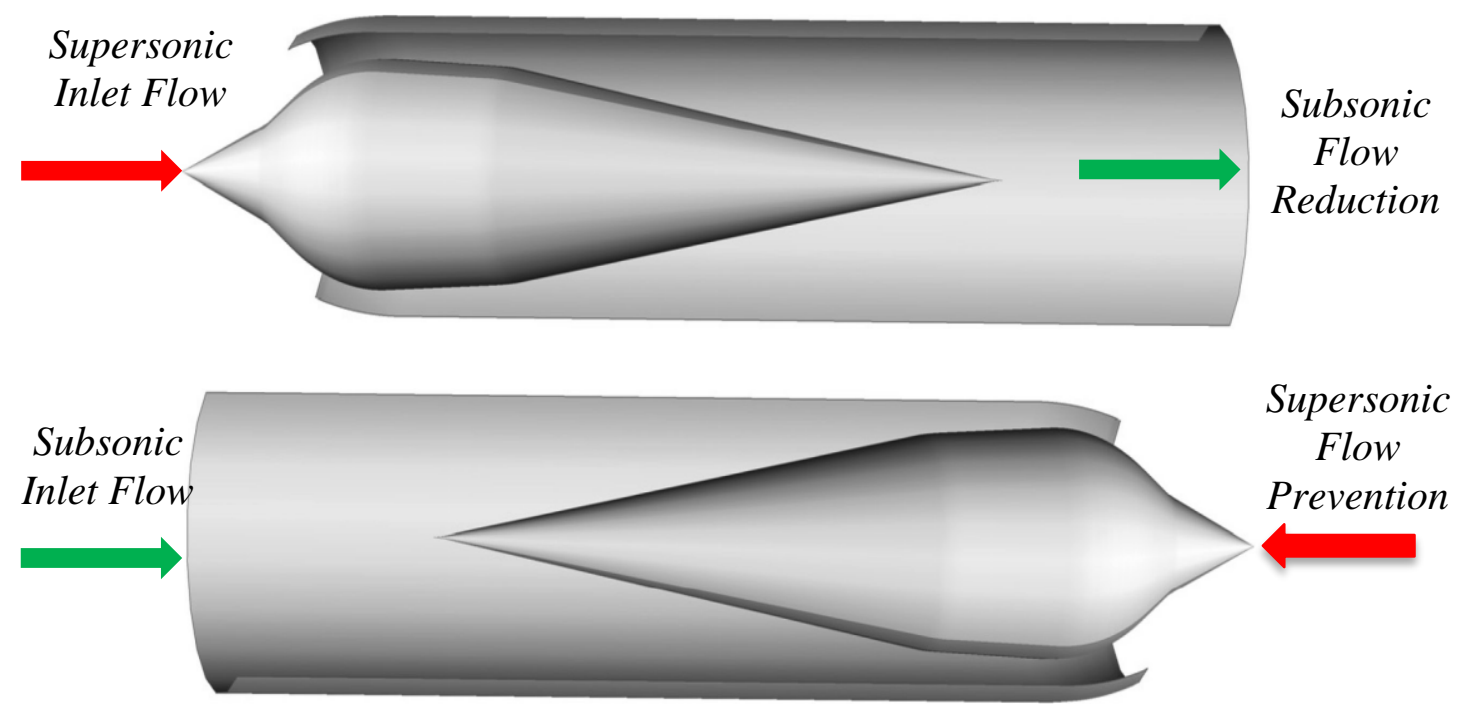

Figure 10. Inlet depictions-Top: aircraft propulsion inlet, Bottom: RDE inlet application 
Shockwave propagation through the inlet poses a problem to the full system. As shockwaves propagate upstream of the inlet flow, it causes serious flow distortion to the incoming inlet flow. In addition, the high pressure shockwaves temporarily prevent the inlet flow from maintaining its forward flow rate. Depending on the severity of the shockwave feedback, it could even cause long-term physical damage to the compressor components farther upstream. Shockwaves are generally undesirable, as they lead to a stagnation pressure loss. Shock losses can often account for a significant portion of pressure losses in a turbomachinery analysis. Since the efficiency is driven by the pressure ratio, any loss to the pressure results in a loss to the efficiency. However, shockwaves are helpful for generating a higher mixing rate, which is a primary purpose of the inlet. The balance of these considerations is addressed in this work. 


\subsection{Problem Definition}

The engineering design problem at hand is quite clear: design an inlet that improves the operation of a RDE and reduces inlet pressure loss. This is a multi-faceted problem, which involves several main issues with inlets. However, even if there are improvements in some aspects of the design, it can lead to downfalls in other aspects of the design. Upon the passing of the detonation wave, the inlet flow is temporarily blocked from the high pressure detonation front. This results in backflow through the inlet, as opposed to the necessary forward flow to refuel the combustor. The phenomena is demonstrated through the illustration in Figure 11. As mentioned previously, the current inlet designs cause high pressure drops across the inlet stage, and also produces non-ideal mixing of the fuel and oxidizer streams. Since the induction and chemical kinetic time scales are much less than the characteristic flow time scale, mixing is challenging in the detonation environment. In other words, the Damköhler (Da) number is quite small, which is the ratio between the chemical length and the fluid dynamic length parameters [18]. The Da number provides a good description of the characteristic times, and hence how well mixing occurs. It is important to note that although hydrogen and air are the primary fuel and oxidizer combination discussed in this work, it is more appropriate to address them in general terms, as the fuel and oxidizer. Other common fuels used in RDE research have been ethylene, methane, acetylene and even liquid hydrocarbons. Standard air, enriched air and pure oxygen have also been used in combination with these fuel options. 


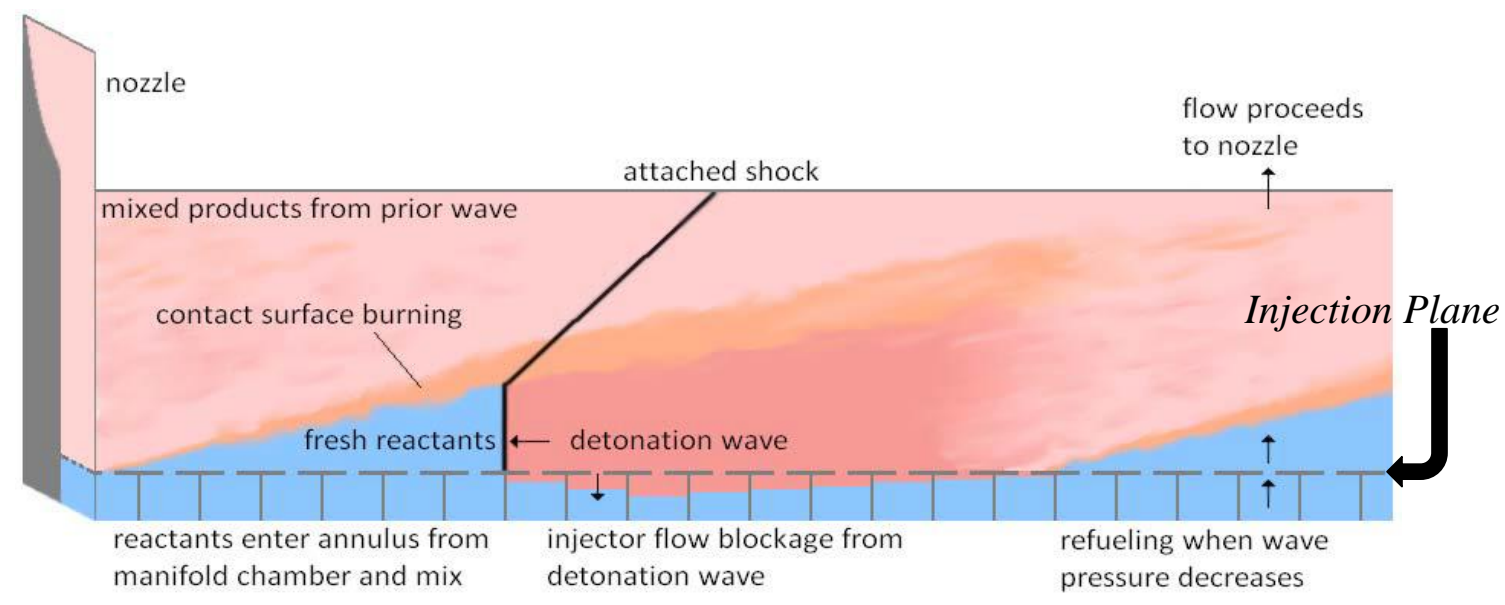

Figure 11. Illustration of inlet backflow phenomena [19]

Outlining the main issues and tasks of an effective inlet design is important for guidance to the project. Without these problems outlined, it would be difficult to evaluate the inlet design. It is of value to address some qualities of a good inlet design, and identify how each of those metrics will be measured. Four critical aspects that are considered and assessed throughout the course of this study are compiled to create a roadmap for the design work at hand:

1) A good inlet design will induce a low pressure drop at the air intake. This can also be described by $D$, which is the diodicity of the design [20]. The diodicity is defined as the ratio between the pressure drop in the reverse flow direction, $\Delta P_{r}$, and the pressure drop in the forward flow direction, $\Delta P_{f}$, as shown in the Equation 1 below. Increasing the diodicity will theoretically improve RDE performance. This is especially relevant for the spade diode design discussed in Chapter 4. But it can be measured for any inlet design that is conceived.

$$
D=\frac{\Delta P_{r}}{\Delta P_{f}}
$$


2) Pressure feedback into the intake manifold will be reduced below the baseline levels. This is measured by the pressure ratio, which compares the peak plenum pressure to detonation wave pressure. Any amount of improvement from the baseline design will be considered a successful step in the right direction.

3) Simple mechanical and geometric design. A design incorporating moving parts is not ideal, because it will reduce the life cycle of its components. A complex geometric design will also be undesirable, because it will warrant difficult manufacturing and machining processes.

4) Size is a crucial design parameter. While this is not a critical driving design consideration for power generation applications, it will be important for aeroderivative uses. It will be especially true for RDEs that may be integrated into a propulsion device for aerospace applications, in which size is a more critical design variable.

The inlet design is complex since there are two separate flow streams that deliver the fuel-air mixture. The primary concern is with the oxidizer inlet. For power generation applications, the greatest penalty will come from losses to the air stream that is being compressed upstream in the compressor. Changes to the oxidizer injector geometry is the main focus of this work, since it is more costly than fuel injection losses. In order to achieve the appropriate equivalence ratio $(\varphi)$, the mass flow rate $(\dot{m})$ of air entering the combustor is significantly more than that of the fuel. Any pressure drop across the inlet is a decrement to the combustor's intended pressure gain. Keeping this pressure drop to a minimum is therefore critical. Since there are multiple flow regimes present in the combustor, it makes the inlet design problem ever more vexing. Flow ranges from approximately incompressible at the inlet manifold, to highly compressible at the detonation region. Since combustion is occurring and the flow is reacting, it adds a degree of difficulty as well.

Fuel injection is the other obstacle for the inlet manifold. Since fuel feed pressures and flow rates will be notably much less than the feed pressure for the air inlet, it is more 
susceptible to the backflow effect. The way the inlet promotes mixing and distributes the fuel for the combustion process is crucial. The baseline inlet has been determined to be vulnerable to instabilities from the detonation. Figure 12 shows that the inlet will become unchoked due to the detonation events in the annulus. The unchoking of the inlet also makes way for the combustion products to propagate through the inlet following the peak of the detonation wave. This will have a direct impact on the efficiency of the combustor. If mixing is inefficient, fuel will remain unburnt and cause a penalty to the efficiency. In addition, it will eject excess hydrogen (or hydrocarbons) from its exhaust and become a pollutant to the atmosphere. The inherent difficulty with mixing is there will always be a notable amount of pressure drop required to promote it. When considering two ideal and perfect gases, in order to perfectly mix them there must be entropy generation. Completely disregarding any inlet pressure losses, there still remains a decrease in the system's pressure that is caused by mixing. From the principles of thermodynamics, with an increase in system entropy must come a pressure loss. By following an isothermal line on a T-s plot, as entropy increases, it is clear that pressure decreases.

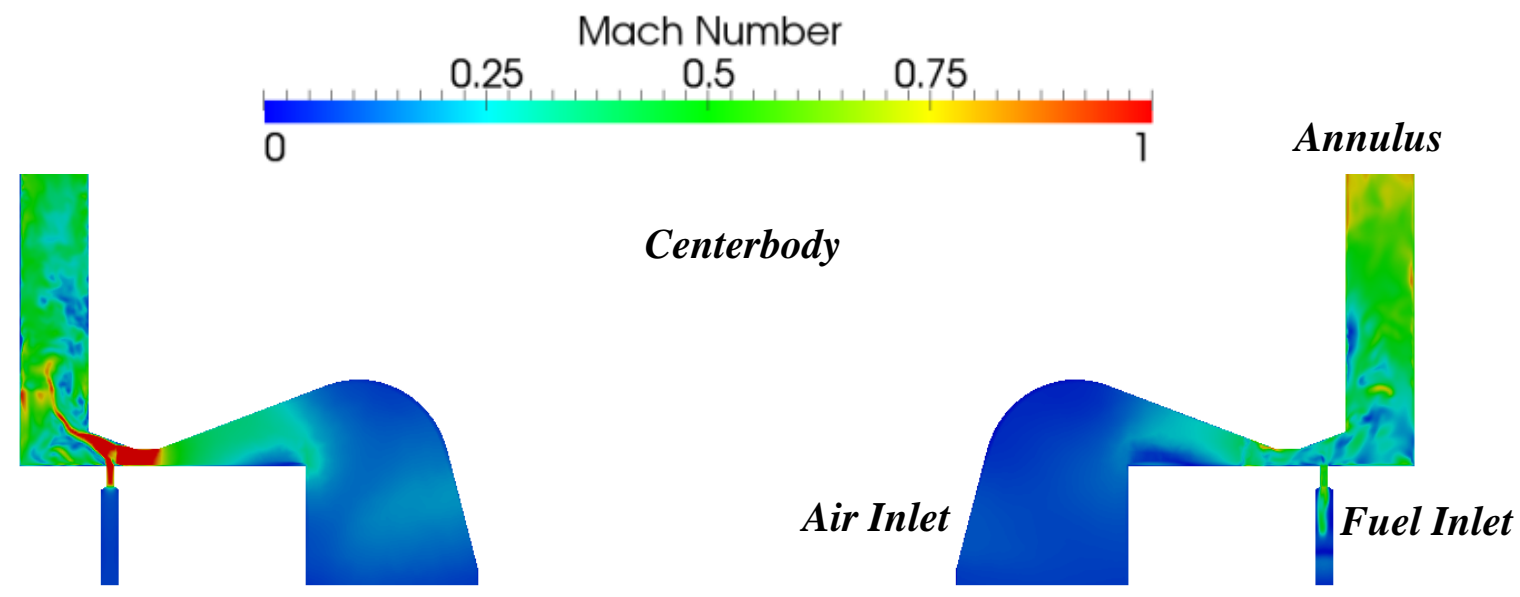

Figure 12. Mach number contours, after 0.5 wave revolution has passed by (left), and just after the wave has passed by (right) [21]

The relationship between pressure loss and mixing efficiency is crucial to understand. Mixing requires a reduction in length scales, which often means stretching of 
a material surface. While forced mixing is often necessary to achieve appropriate levels of homogeneity, there are losses that accompany the process of forcing the mixing behavior. The pressure and mixing ability are intimately related, and have an inverse correlation. Generally, high mixing efficiencies indicate a high pressure loss. One method of increasing mixing efficiency is to create high flow velocities, which results in a high pressure differential. This drives mixing efficiency higher, but sacrifices the pressure loss. It is desirable to find a healthy compromise between the two parameters. The challenge lies in enhancing the mixing through techniques that do not increase overall pressure loss significantly. The compromise often lies in what parameters are more crucial for design. For instance, if thrust or specific fuel consumption (SFC) has a high design target, then it may be more important to focus on improving the pressure loss [22]. The obvious thought may be to inject air purely axially, rather than sharply turning the air pathway to inject radially. However, the turning generates high vorticity in the air stream. It is also possible to utilize passive solutions to increase vorticity in the flow, with things like trip strips and other turbulence generating devices. This is important to improve the mixing between the air and fuel. Otherwise, the air and fuel may just be directly impinging jets, which has less sufficient mixing than a configuration with flow turning and vorticity. 


\subsection{Objectives}

The objectives of this study were to test novel inlet design concepts for the RDE and develop a better understanding of how the characteristic operation of a RDE's internal flow due to detonation affects a chosen inlet geometry. Various inlet concepts were conceived and manufactured to evaluate in the inlet test rig. Evaluation of variations of the current baseline inlet was also performed to determine its potential for improvement. By equipping the test rig with multiple diagnostic methods, such as pressure measurements and high-speed video, critical data was gathered for each inlet that was considered. The goals of this study are to demonstrate several important improvements for inlet designs tested:

1) A reduction in pressure drop from the current baseline inlet. The current static pressure differential across the inlet, with the smallest air gap, stands at around $90 \%$. This leaves significant room to improve on this loss factor.

2) The heightened prevention of backflow from the detonation wave within the combustion annulus. This will be quantified by the pressure ratio for the inlet flow [23]. This defined pressure ratio, which is given below, is indicative of the backflow effect on the inlet performance. It is different than typical pressure ratios defined in other turbomachinery application. It is specific to this application for inlet testing, where $P_{02}$ is the maximum plenum pressure, and $P_{01}$ is the maximum detonation pressure measured in the channel. The result is a percentage of the peak detonation pressure, where 0.04 would correspond to $4 \%$ of the peak pressure. The target is to decrease the pressure ratio $(P R)$ to the highest extent, and this is shown in Equation 2.

$$
P R=\frac{p_{O 2}}{p_{O 1}}
$$


3) The amount of time that the inlet flow is overcome by the feedback of the combustion products should be as short as possible. The less time that the pure inlet mixture is overwhelmed by the propagation of hot combustion products, the better performance potential the RDE has. The feedback of combustion products into the inlet can overcome the fresh inlet flow so much that it can cause an instability and loss of detonation [24]. This is critical because during the periodic operation of the RDE, any given location on the inlet will have a detonation wave passing up to 5000 times per second. Therefore, if the inlet flow is blocked from the post detonation zone products, it will never have time to fully recover to its full flow potential. Two time values are defined and quantified for each inlet. The interruption time and recovery time are main objective points for this work, which are defined in more detail in later sections.

4) Improved mixing efficiency $\left(\eta_{m}\right)$ between the fuel and oxidizer, where:

$$
\eta_{m}=\frac{\dot{\mathrm{m}}_{\text {fuel,mixed }}}{\dot{\mathrm{m}}_{\text {fuel,total }}}=\frac{\int_{A_{\alpha=0}} \alpha_{L} \rho u d A}{\int_{A_{\alpha=0}} \alpha \rho u d A}
$$

For this expression, $\dot{m}_{f u e l, \text { mixed }}$ is the mass flow rate of fuel that is mixed, $\dot{m}_{f u e l, \text { total }}$ is the total fuel mass flow rate for the system, and $\alpha_{L}$ is the local mixing quantity. In Equation 3, the mass flow rate term is expanded out into the density $(\rho)$, velocity $(u)$, and differential area $(d A)$. The local mixing quantity is shown by the piecewise formula in Equation 4, in which the stoichiometric mixing quantity term $\left(\alpha_{\mathrm{s}}\right)$ is introduced. This has been adopted from Doster et al. [25] and applied to this work.

$$
\alpha_{L}=\left\{\begin{array}{cc}
\alpha, & \alpha \leq \alpha_{s} \\
\frac{\alpha_{s}(1-\alpha)}{1-\alpha_{s}}, & \alpha>\alpha_{s}
\end{array}\right.
$$

The mixing quantity $(\alpha)$ can be more simply expressed as seen in the equation below. For the sake of generality, the oxidant will be referred to as the oxidizer. 
However, in all cases referred to in this study, the oxidizer is always air. In Equation 5, $f$ is the fuel-air mass ratio. Equation 6 defines the mixing quantity in terms of the equivalence ratio $(\phi)$, and the stoichiometric fuel-air mass ratio $\left(f_{s}\right)$.

$$
\begin{gathered}
\alpha=\frac{\text { mass of } \text { fuel }}{\text { mass of fuel }+ \text { mass of oxidizer }}=\frac{f}{f+1} \\
\alpha=\frac{\varphi f_{s}}{\varphi f_{s}+1}
\end{gathered}
$$

Although there are no experimental measurements of mixing ability of the inlets, some CFD results are revealing about the mixing capability of the inlet.

An improved inlet will minimize the acoustic coupling between the detonation annulus and the inlet plenums, which is detailed later in the section. This will isolate the inlet from the combustion annulus so the interaction and communication between the two is minimized. The more the detonation from the annulus communicates with the inlet plenums, the less adequate the refueling capability of that inlet becomes. Conceptualizing an inlet design that is well isolated from the detonation annulus is an important first step in improving the inlet designs. Pressure drop can conceivably be easily reduced by reducing the restriction ratio at the inlet's minimum throat area. However, in doing that, the inlet receives unacceptable amounts of feedback from detonation that makes the process too unstable. If the geometry can be tuned so that it has high isolation potential, forward steps can then be taken to reduce the amount of downstream restriction. 


\section{Chapter 2: Review of Literature}

Although the RDE's inlet is one of the more demanding areas of research, little experimental work has been done to test new design concepts. Since RDE technology is fairly new on the scene, much of the research focus has been testing its operational map and understanding the complexity of the system. Significant work has been done in the way of numerical modeling for RDE inlets. The experimental and operational work all seems to point back to the inlet as a bottleneck in the system design. Without an efficient and successful inlet design, the entire system suffers. The ability of the inlet to effectively mix the fuel and oxidizer will drive the combustion efficiency, which will then also drive the emissions levels.

Perhaps the most relevant study for the inlet design was a set of computational studies performed by a group from the Naval Research Laboratory (NRL). Schwer et al. [26] compared the effect of various inlet geometries on the operation of the RDE. The first set of injectors examined were slot micro-injectors. Rather than the baseline circular shaped fuel inlet holes, the slots presented a new concept in terms of inlet geometry. The study also compared angled injectors to the standard $90^{\circ}$ vertical injectors. They tested injectors on a positive 20 and $40^{\circ}$ angle, and then negative 20 and $40^{\circ}$ angles. The injector shapes were also modified. A cavity slot (shown in Figure 18), nozzle slot, and diode slot were all innovative shapes to the injectors that were tested. Based on their computational efforts, none of the alternate injectors seemed to improve the performance of the inlet. In fact, the majority of alternative designs showed an increased amount of pressure feedback into the inlet manifold. 
Continuing their efforts in computational research, Schwer et al. [27] chose to perform computational analyses of a RDE for some cases without injectors completely. Rather than modeling the discrete injection scheme, they represented the injectors as two different types of models. One approach was an idealized source term injection, and the other was an idealized boundary condition, which combined the effects of the mixture plenum and micro-nozzles. Of course, the two idealized cases run based on many assumptions, one of which is that injection flow is instantly and perfectly mixed prior to combustion. They found that the idealized imposed boundaries gave very similar flow fields, but quite different quantitative results. The boundary condition case produced significantly higher pressure, mass flows, and overall performance than the source term. The 2-D representation revealed a complex flow field, with strong interactions between the transient detonation wave and the fuel injectors. However, performance was matched closely with the two ideal cases. The results from this work produced important conclusions about the dynamic response of the injectors to the combustion within the annulus. It revealed that the stability of the RDE can hinge on the behavior of the injection scheme.

Fotia et al. [28] developed methods for simulating the environment of a RDE detonation channel. The intent behind these studies was to create a device with similar geometric constraints as the RDE. Multiple interactions were measured from these devices, including: pre-mixing effects, detonation interaction with inlet plenum, and feedback and flashback tendencies. Through the use of detonation tubes as ignitors, a detonation wave is distributed through the channel. By altering nozzle throat size, it was found to have an impact on reducing the flow propagation into the inlet plenum. Naples et al. [29] quantified fuel injector response to detonation in the plenum, along with plenum pressures and velocities. They discovered that the frequencies of the plenum pressure, channel pressure, and plenum velocity are not necessarily the same, but are related through common harmonic frequencies. They also measured an appreciable change in the fuel flow rate as a result of the detonation wave within the channel. Since fuel flow rate is altered from the detonation, it has a significant impact on the local 
equivalence ratio. This directly affects the efficiency of combustion and many other critical variables, like local temperatures and pressures. This emphasizes the need for a fuel injection scheme that is able to effectively resist these transient changes as a result of the detonations.

Similarly, Anand et al. [30] characterized the air and fuel plenum responses in a fired RDE. These tests were carried out on the original slotted inlet design for the RDE, discussed shortly hereafter. This geometry is fairly simple, with flat slots used as the injectors. By correlating the pressure-time response of the plenums to the detonation channel, the spatial and temporal variation of fluctuations in the plenums can be determined. An underlying, lower fundamental frequency was uncovered in the air plenum. This was shown to closely match the Helmholtz frequency of the plenum geometry. Therefore, it is not surprising to see a frequency at this level present in the plenum. It was postulated that the detonation wave excites the plenum to act as a Helmholtz resonator. The magnitude of the response in the fuel plenum was seen to be less than the air plenum. The fuel plenum measures pressure fluctuations in magnitude of around $20 \%$ of the total pressure exhibited by the detonation, while the air plenum sees between 45 and $70 \%$ of these fluctuations. This is because the fuel plenum static feed pressures are typically lower than the oxidizer plenum feed pressure, and as a result is more susceptible to influence from the detonations. The results unveiled a need for more detailed equations to determine the resonance frequency for complex inlet or plenum geometries.

The local change in equivalence ratio that is caused by the upstream propagation of the detonation wave can have a significant impact on the RDE operation. From CFD simulations done at NETL, in addition to experimental data, it has been seen that the current inlet design is susceptible to wave bifurcation and unpredictability of the bifurcations. The change in equivalence ratio occurs when a small area of the fill region is obstructed, which leads to locations of highly lean or rich equivalence ratios. This phenomenon and the inlet design may contribute to the bifurcations that are observed. 
This theory was supported by a research group at the University of Texas at Arlington (UTA). Braun et al. [31] tested two RDE configurations in which swirled injection was used. One geometry pre-mixed the fuel and oxidizer prior to detonation, which can be seen in Figure 13. This helped create a more homogeneous inlet mixture. The second geometry mixed the fuel and oxidizer in the detonation chamber. However, there were no measurements in place to indicate the feedback effect on the inlet. The important takeaway from these experiments though is the lack of formation of multiple detonation waves in the pre-mixed configuration. Perhaps the critical factor in those findings is the fuel-oxidizer mixture that is being introduced to the detonation chamber is more homogeneous and better mixed than alternate inlets.

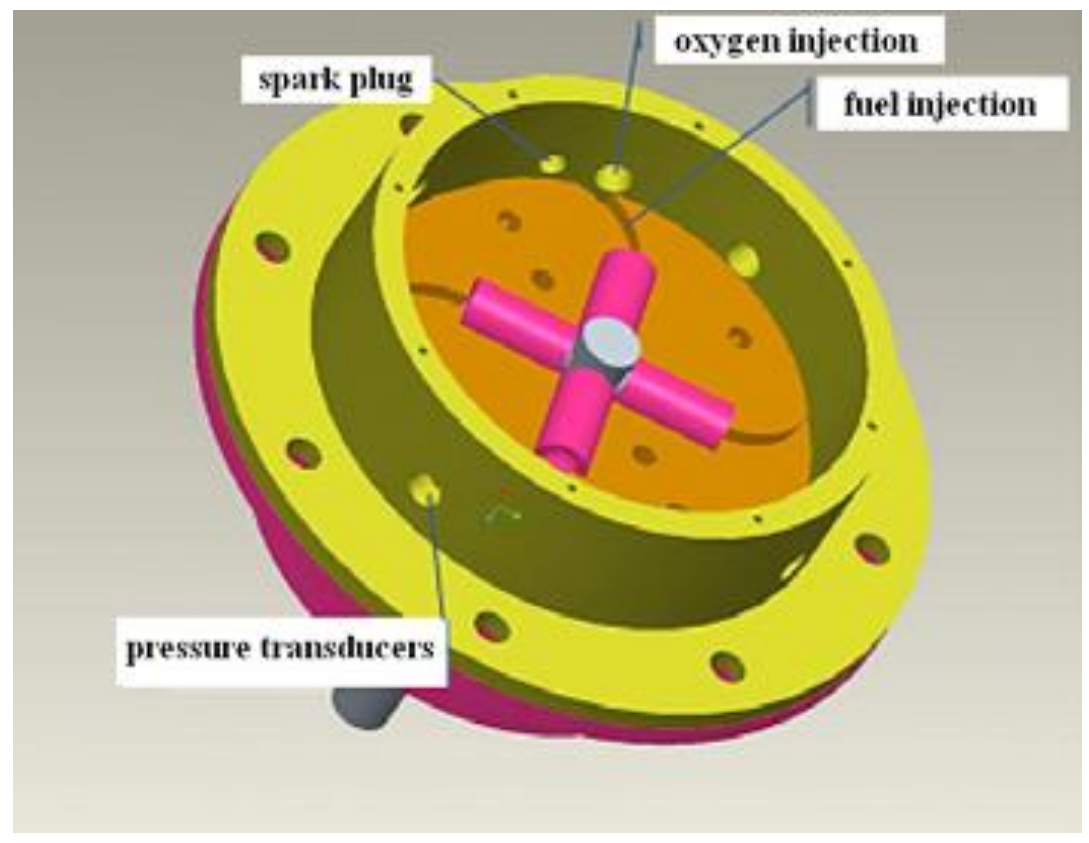

Figure 13. Swirled injection chamber [31]

The same research group from the University of Texas at Arlington performed some RDE inlet work in the past few years. Braun et al. [32] performed experimental studies on a fluidic type of fuel injector. With this configuration, a parametric study is done on a square-shaped cavity to characterize its response to detonation combustion. 
The valves were mounted perpendicular to the direction of the detonation wave passing, just as they would be in a realistic RDE environment. By altering pressures, geometry, and fuel sources, the feasibility of this fluidic valve was examined. It was found that this valve holds strong possibility for successful use in a RDE. Since the rig that this was tested in only fired one detonation, there was no way of matching the actual RDE frequencies or simulating multi-wave behavior. It responded to characteristic times of the detonation tube that match around a $5000 \mathrm{~Hz}$ frequency.

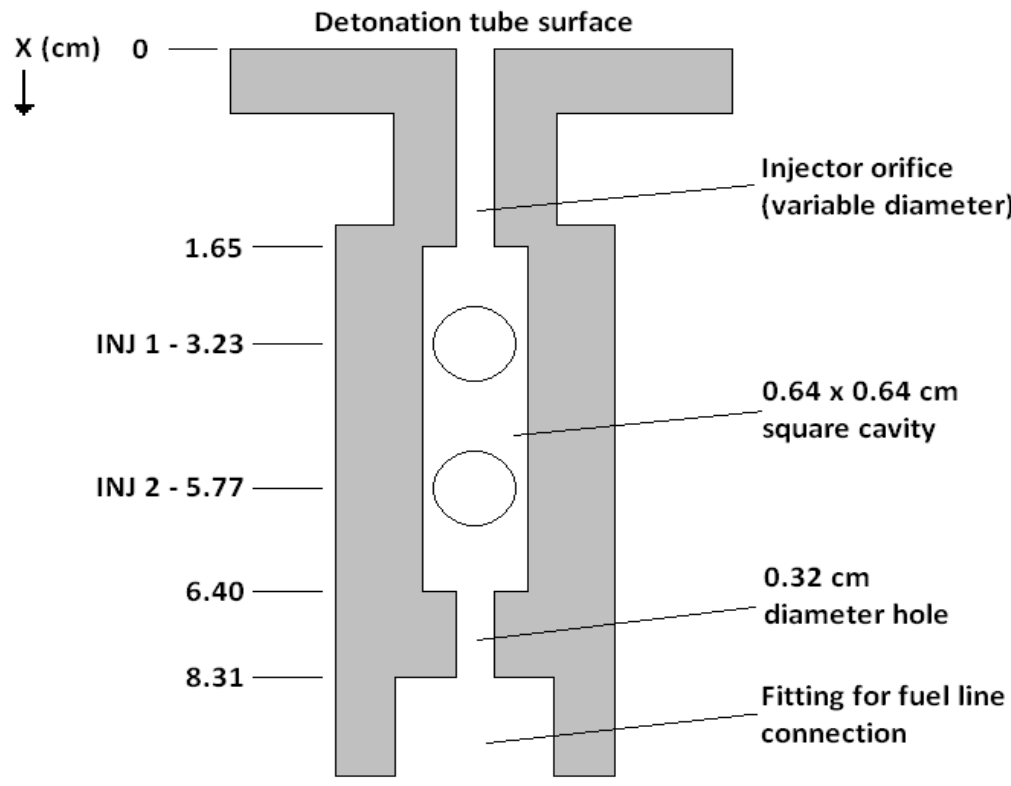

Figure 14. Cavity-type fluidic valve [32]

This work was then further developed by Peace et al. [33]. Their results revealed that the length of the cavity is important in reducing the presence of reflected shockwaves within the injection area. From the pressure traces in the injector, they were able to accurately trace and monitor the shock reflection behavior. It was shown that a shorter cavity is more conducive to avoiding excessive shockwave reflections in the inlet area, which leads to undesired pressure fluctuations.

This experimental study can again be closely compared with the computational work from Schwer that has already been discussed. By looking at the injectors in Figure 
15 and comparing with the geometry of the injector in Figure 14, it is clear that they are very similar. It is interesting to compare the conclusions from both sets of work. The computational study did not provide much detail about the dynamics of the newly designed injector in relation to the detonation in the annulus. It simply deduced that the new injector did not improve the feedback prevention - it actually indicated worse performance. However, the experimental study showed good results from the new injector. The computational work matched the RDE conditions closely, while the experimental study used non-realistic operating conditions. The UTA work created normalized data to extrapolate the injector's performance to the real RDE conditions. They created a non-dimensional interruption time from which they calculated its relevance to quasi-RDE conditions. This is important to notice for future inlet studies. It may be an indication that performing experiments in an environment that does not match the RDEs may be inaccurate to extrapolate data from. Until further work is done to confirm this, it is difficult to draw any firm conclusions.

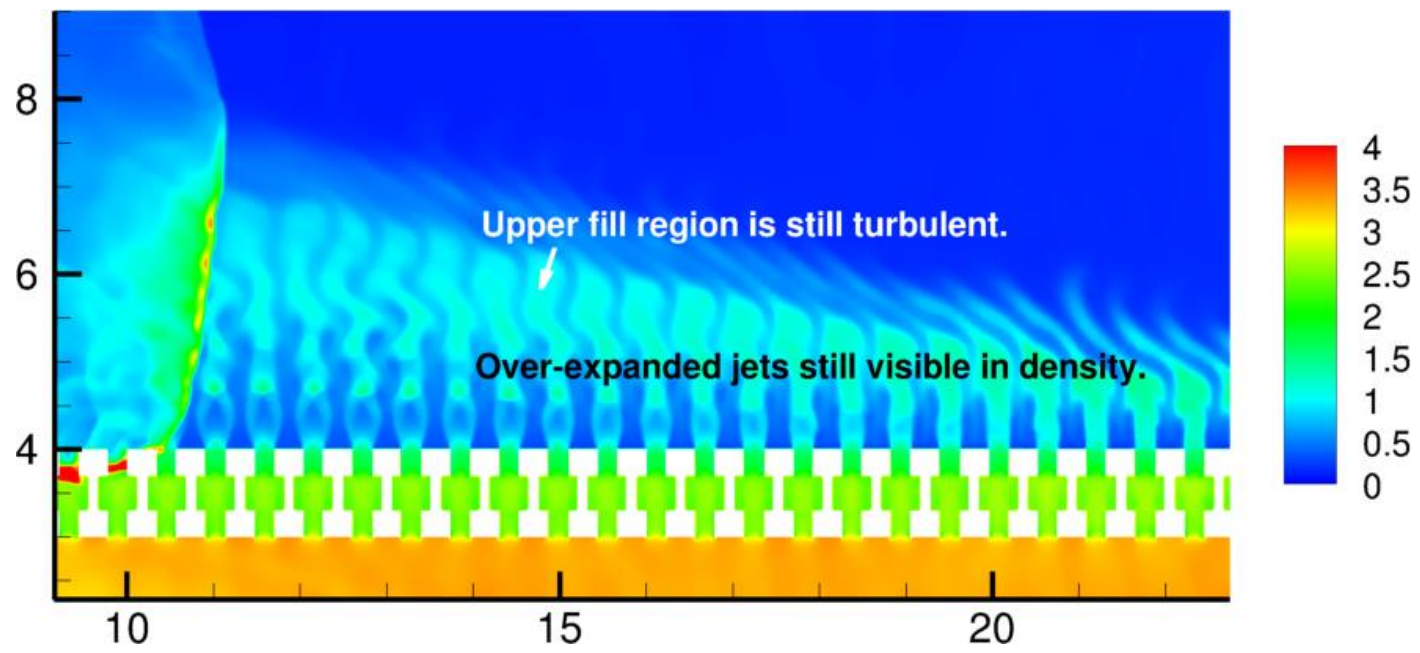

Figure 15. Density solution for cavity-slot injection design [26]

Within the same study referenced earlier, the experimental research group from UTA began to perform tests on a RDE with swirled, pre-mixed inlet conditions. This set of tests provided useful insight into the mixing issues contained with the RDE inlet. Since 
it was determined that a pre-mixed reaction could occur safely and effectively, this gave assurance for the designs discussed herein to have potential as a pre-mixed configuration.

The problem of a well-designed inlet presents itself in other technologies as well. Research has been under way for over 60 years in different arenas for inlet designs. The scramjet propulsion system has seen many issues with its inlet. The scramjet systems parallel RDEs closely. Since they operate under supersonic combustion conditions as well, similar issues of mixing under very high speeds is a cornerstone issue. Devising better methods for mixing and air intake has been the challenge. With supersonic combustion being the driving mechanism behind this technology, it is relevant to the RDE. As the aerospace industry continues to push the envelope for flight speeds, the inlet will continue to be one of the bottlenecks in technical issues. Findings from other research studies can be understood and applied to a certain extent to RDE inlets. For instance, much of the scramjet combustion research has relevant implications to RDEs. A group from NASA Langley Research Center has completed studies examining fuel and air mixing at high speeds, as well as pulsed flow as a method for flow control of inlets. Drummond et al. [34] tested two traditional techniques for fuel injection. These methods include fuel injection from the wall and in-stream injection from struts. Alternate configurations of the wall injection schemes have shown potential for improving downstream mixing.

Among the small group of experimental researchers focusing on RDEs is also a team from the University of Cincinnati. Driscoll et al. [35] recently published some work concentrating on the mixing characteristics of the inlet. Their baseline test geometry was the initial RDE inlet designed by Shank from AFRL. They observed that as fuel injection was moved back farther into the air injection, that equivalence ratio was increased and mixing was enhanced prior to entering the annulus for combustion. They also noticed that decreasing the fuel injection area and increasing its corresponding mass flux contributes to better mixing. However, a change such as this becomes impractical because the losses that it cause outweigh the potential benefits of implementing it. They measured a change 
in the local equivalence ratio near the exit of the fuel injection locations. There was an increase in this local $\varphi$, caused by the greater mixing ability at the outlet of the fuel injector. It is crucial to identify the recirculation region that is created where the air inlet meets the annulus. This recirculation tendency is important to the mixing behavior of the inlet. The more potential the fuel has of reaching this recirculation zone, the greater the mixing efficiency will be. Figure 16 depicts the inlet that this study was performed on. By comparing this with the newer design (introduced earlier in Figure 8), it can be understood why some of the modifications were made. Two of the major differences in these inlets are the relative location of the fuel and air inlets, and the geometry of the air inlet. The more current design features fuel inlets that are placed on a larger radial location (farther away from the center line). This enables the air and fuel some partial pre-mixing ability before entering the annulus. The significant difference in the oxidizer inlet is the curved air plenum that creates the c-d nozzle geometry. This curvature causes the air to turn and tumble into the injection region. This tendency induces high amounts of vorticity, and improves the mixing to an even greater extent.

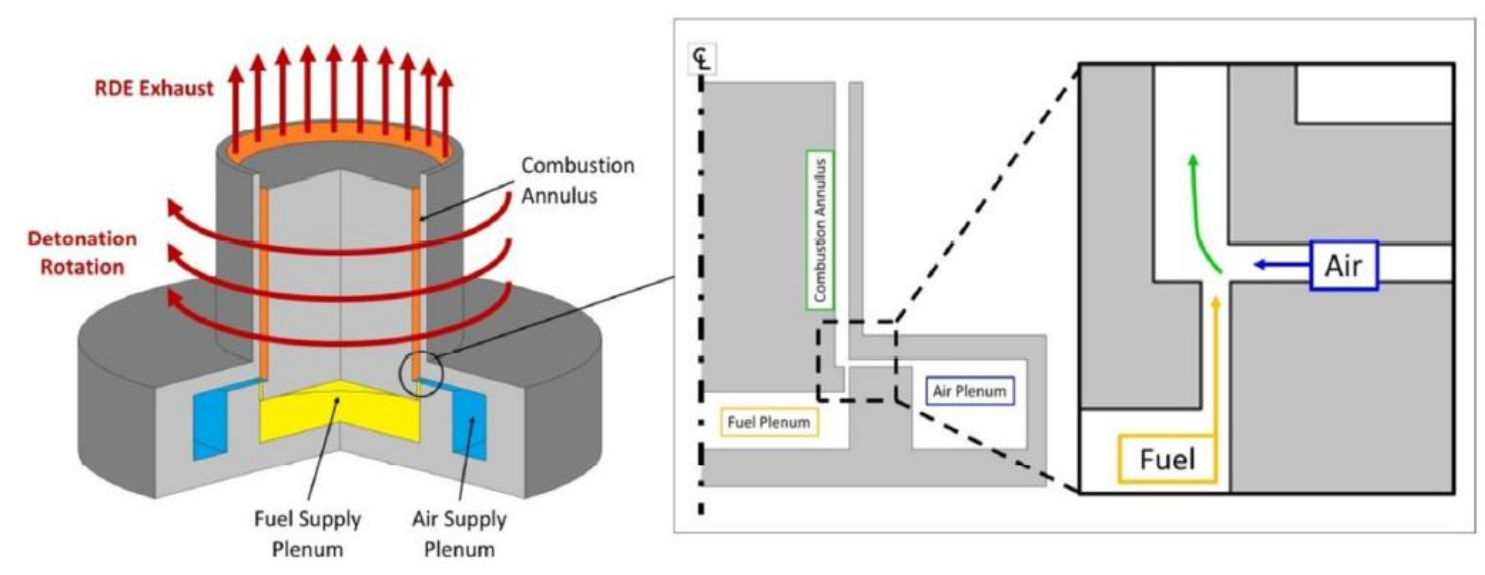

Figure 16. Original inlet design from AFRL, Shank et al. [36] 
Mixing characteristics have been studied for numerous applications. One critical focus is on high-speed mixing in supersonic environments. Hariharan et al. [37] performed computational studies on various shaped injectors. They examined four different shapes. The cross sections were circular, wedge, diamond, and chevron. By using total pressure loss and mixing efficiency as the two main performance measures, the Chevron injector outperformed the other three injector shapes in its mixing efficiency while being comparable with the total pressure loss of the other injectors. This indicates that choosing a specific injector shape alternative to a typical circular cross section may provide benefits to their mixing capability. The chevron injector caused the inlet flow to have better spreading throughout the cross flow and allow for more diffusive mixing. Surprisingly, the circular cross section performed as a second to the chevron injectors. This means that not all uniquely shaped injector geometries are an improvement, since the diamond and wedge injectors underperformed the common circular. Russo et al. [38] showed that for higher fuel flow rates (corresponding to higher fuel jet velocity), the operational steadiness of the RDE is improved. The exit velocity of the fuel injection is therefore crucial to how well the reactants mix.

Bobusch et al. [39] studied fluidic devices for the applicability towards pressure gain combustion devices (wave rotors, pulse detonation combustors, pulsed combustors). A good history and background of fluidic devices is provided, which lends helpful insight into the original motivation and details of the designs. This work combined computational with experimental results to give a hybrid blend of data from each perspective. The bulk of the research was dedicated to a fluidic switch. 


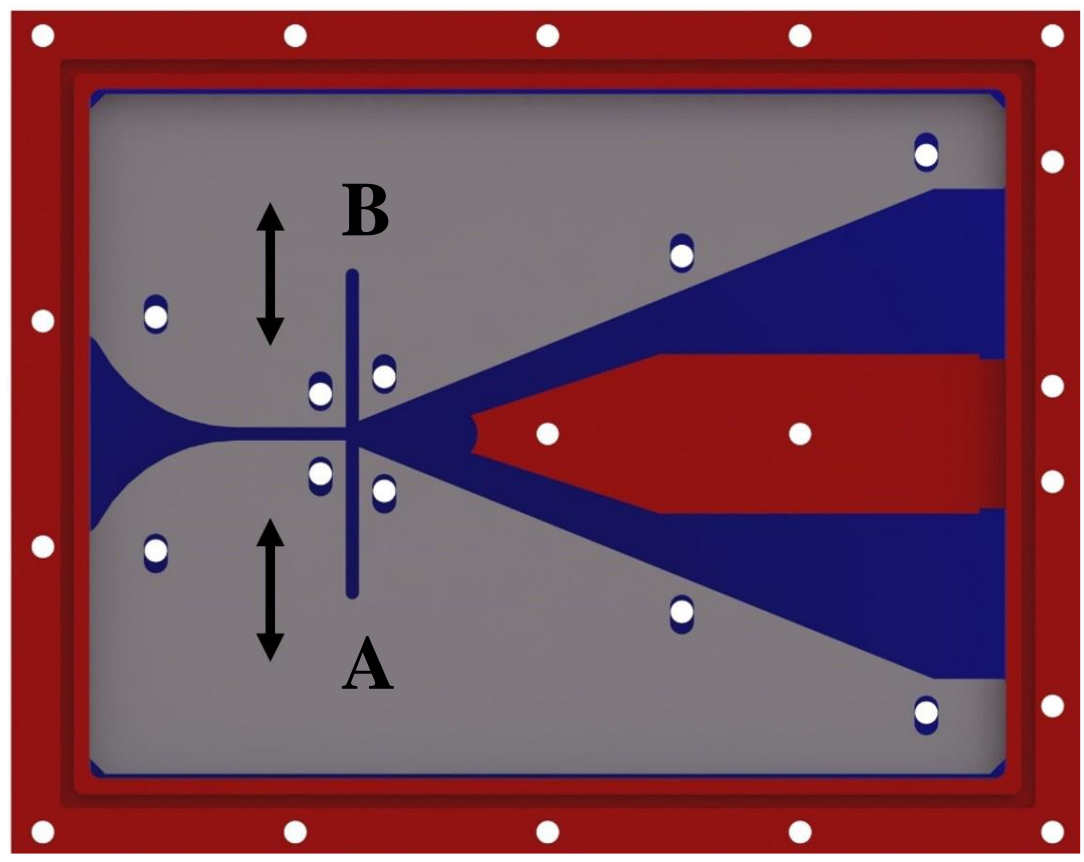

Figure 17. Fluidic switch, Bobusch [39]

The fluidic switch takes advantage of the Coandă effect. By injecting flow perpendicularly with respect to the mainstream flow through the blue slots, the mainstream airflow attaches to one of the two diverging passages. The design is represented in Figure 17. This is a clever method of flow control; however, the control valves only respond within a few milliseconds, which is driven by the length of the tube between points $\mathrm{A}$ and $\mathrm{B}$. Because of the slow response time, this method would not be feasible in the RDE. However, the Coandă effect is a useful phenomenon that could be leveraged in some ways for future RDE inlet studies. This phenomena is often used in boundary layer control type applications for aircraft control surfaces. While this switching is not done actively, the Coandă effect essentially makes the fluid "sticky", and it has the tendency to attach itself to walls. Through this means, the directionality of the fluid can be more easily controlled.

The two issues that Bobusch's work addressed were the poor mixing of other designs and the target of an effective back pressure valve that can operate at high 
frequencies. Both of these qualifications are relevant to the RDE inlet studies. Another pillar of the fuel injectors examined was fluidic oscillators. Upon numerical simulations as well as experimental studies, the fluidic oscillators proved to be a probable candidate for an improved fuel injector. The results also revealed that with detailed design, an operating frequency of up to $10 \mathrm{kHz}$ can be reached. The experimental results were found from a water test rig. Therefore, much extrapolation needs to be performed to account for the change in working fluid to a gaseous substance. For an RDE, these oscillators could prove very useful. Rather than creating an internal feedback within the oscillator itself, the feedback could be driven by the detonation events. In this case, the oscillator could work at whatever frequency the detonation is driving it at. There are many limitations and considerations for this theory, but it is worth exploring.

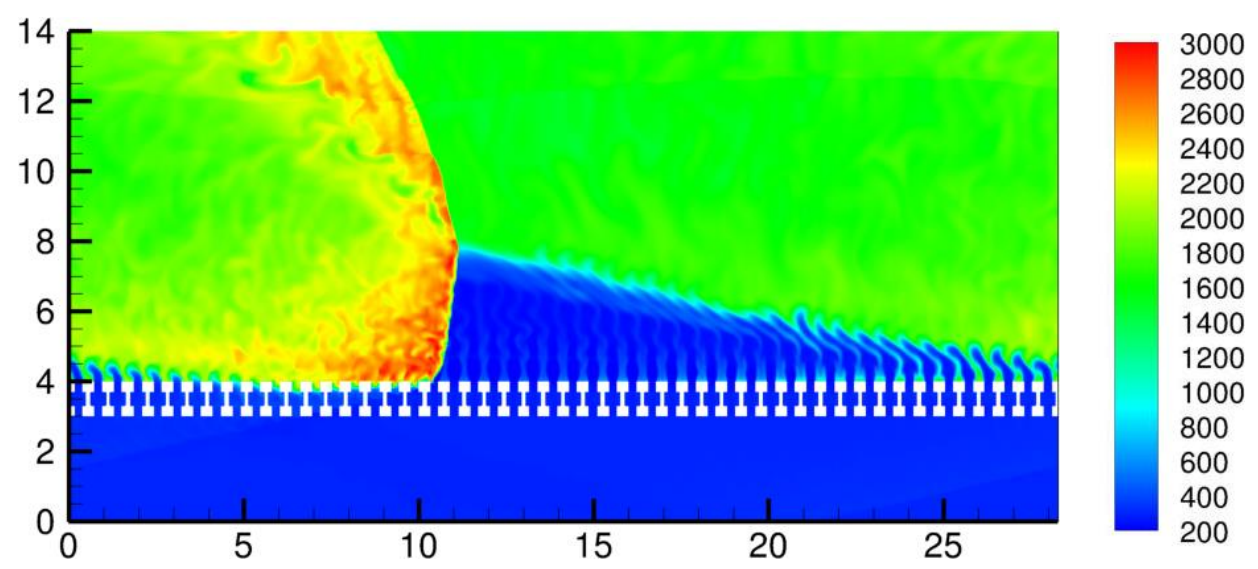

Figure 18. Temperature (K) solution for cavity slots-wave propagation is left to right [26]

After reviewing literature on other fluidic devices, Bobusch selected a scroll diode for testing as it was believed to have the most potential for improvement. This design can be seen in Figure 19. Test results revealed that the diode met most of the top requirements for improvement. The diodicity as a function of Reynolds number was measured. The scroll diode provided a low pressure loss coefficient and a notably high diodicity. It did not successfully reflect all pressure waves. It is believed that altering some of the features of the diode tested may lead to better performance. Changing the diameter of the barrel, or the size of the cusp will dramatically impact the performance of 
the diode. The size of the reflection cusp will have a serious impact on the ability of the diode to reflect pressure waves. Moving the focal point of the cusp to a location farther upstream could alleviate pressure wave propagation as well. Through a parametric study, the optimal conditions for this design could be attained quite simply.

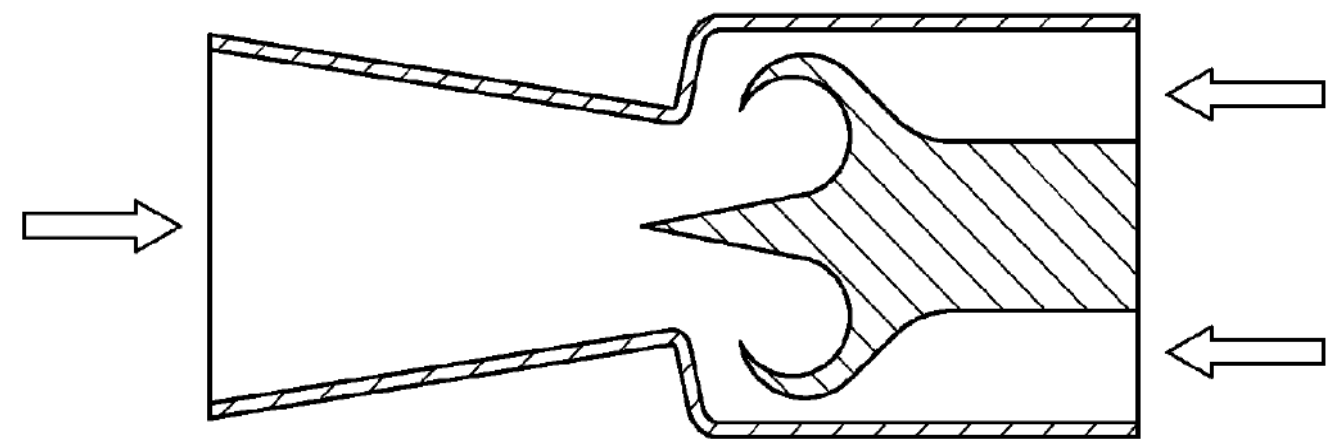

Figure 19. Scroll diode with 'tulip like' geometry on centerline [39]

The development of a pre-mixed inlet is hypothetically the most effective design for an inlet. A pre-mixed inlet removes mixing effects from the combustion dynamics. Andrus et al. [40] designed a small test setup to simulate RDE behavior in which premixing was done for the feed flow. The key lessons taken from this study was the impact that pre-mixing the inlet flow has on flashback tendency, shock propagations and flow velocities. Utilizing a free expansion nozzle configuration, like the one shown in Figure 20 , some of the tests showed that flashback into the upstream feed location of the combustor is a serious concern. The flashback led to detrimental effects on the test setup. A positive outcome from the tests indicate that pressure waves from the detonations in the channel were not recorded traveling into the mixing chamber. This means that no shockwaves were visualized in the mixing plenum, so feedback was limited.

Observations were also made that showed a decoupling effect of the combustion from the pressure waves. This could have implications to the design goal of the inlet. It seems that there may be two different regimes of flow reversal to prevent (bulk fluid region of 
combustion products and pressure waves that result from shockwaves). These different regions would then require different design approaches. The free expansion nozzles used for testing in the experiment are shown below in Figure 20.

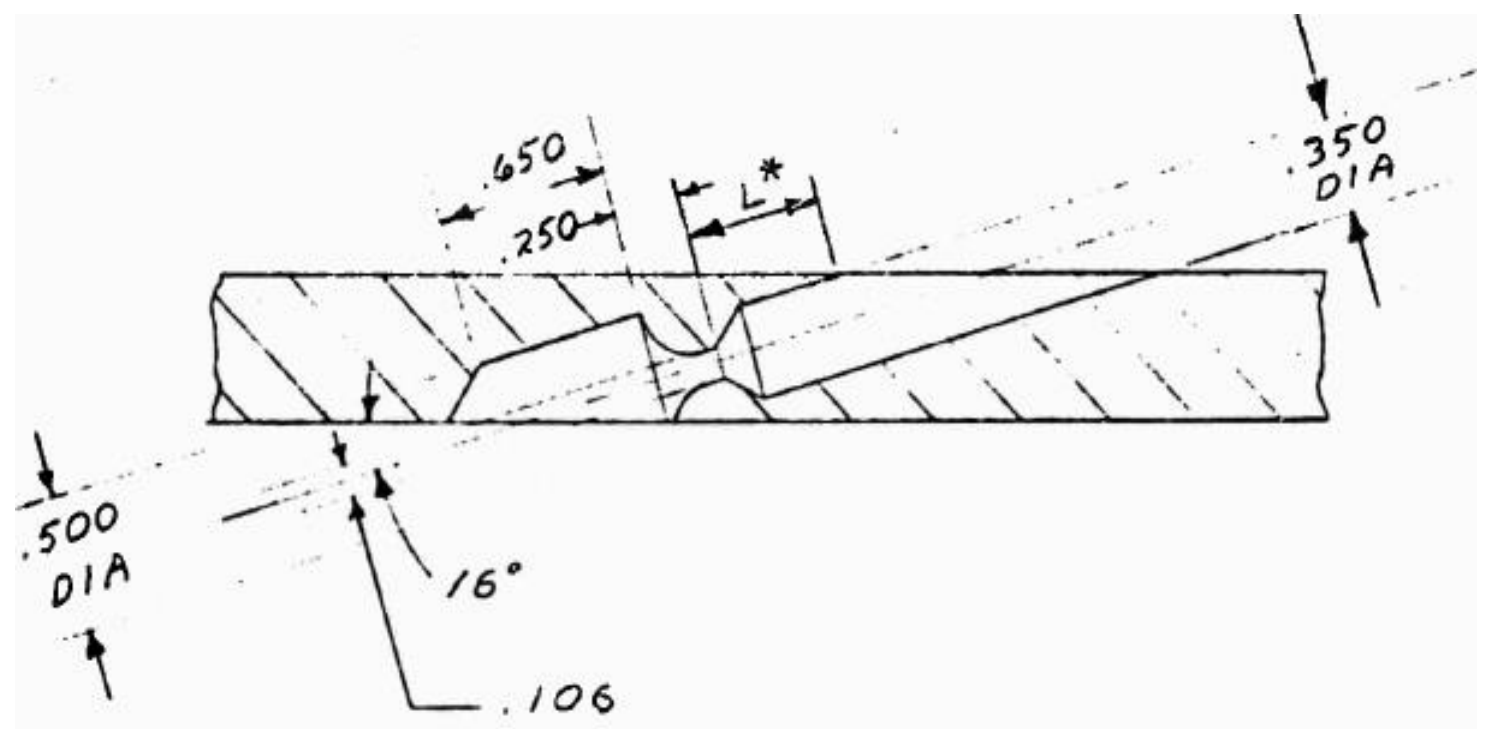

Figure 20. Free expansion nozzle, first proposed by Barakauskas et al. [41]

The findings from the final pre-mixed design tests reveal that the primary inlet design criteria for a pre-mixed RDE differ from those of a non-premixed RDE. The first set of requirements that an inlet must meet for a pre-mixed RDE are its appropriate quenching distances, velocity gradients, and penetration lengths. Tuning these parameters are necessary to prevent flashback first and foremost, and secondary comes the optimization of the designs. It also turns out that the geometries necessary to satisfy the quenching distances become quite small, leading to a high induced pressure drop [42]. This brings the inlet struggle back full circle to one of its main weaknesses.

Another similar approach at characterizing the flow interactions due to detonation was performed by Burr et al. [43]. A linear detonation rig was devised in which cross flow was injected into a channel. A PDE introduced detonations to the channel section. The cross flow, which was an approximation of RDE injectant flow, was studied for reignition characteristics. The reignition appeared to be a combination of several factors. 
A determination was made that variations in mixing, as well as pressure waves that are produced from combustion events after the incident shock wave passes by can play a significant role in the detonations present in the annulus.

Turqus et al. [44] have implemented resonators within the intake of internal combustion engines to control valving. Under the cycling of the valves for a two stroke engine, pressurized air columns are used to create a natural supercharging effect. This is accomplished through the usage of a Helmholtz tuning device. Through careful tuning of the system, a higher charge leads to higher output torque for the engine. One Helmholtz resonant frequency theoretically corresponds to a given geometry of the intake ports. The resonator then acts like a mass-spring system. It has been shown to improve the volumetric efficiency of internal combustion engines. The length and area of the neck, in addition to the volume of the resonator cavity are the important design parameters. However, once a resonant frequency is targeted and designed for, it will only match a given operating condition for the engine (i.e. - engine operating speed), although this would not be an issue for a series hybrid stationary power plant. Santana et al. [45] applied the principles of acoustic and resonance directly to combustion. Combustion instabilities can be detrimental to the combustor and its integrated system. By using acoustic liners with damping devices that target certain frequencies of the combustor, the noise and instabilities can be more suitably managed. Common solutions employed using this technique is often cavity resonators, or plates with apertures of specific sizes.

Variable exhaust geometries are studied significantly, especially for aerospace applications. The throat area of nozzles and exhaust transition regions can have a large impact on the RDE overall. Since combustion is occurring supersonically, the effects of an exhaust geometry is not limited to post-exhaust conditions, and they can often impose transient effects upstream. Paxson et al. [46] revealed that an exhaust throat can cause shockwave effects to transfer as far back upstream as to the inlet plane. However, this is not necessarily a negative effect. Strong coupling between the inlet geometry and exhaust restriction is present. In other words, an inlet with stagnation pressure loss across it can 
lead to an improvement in specific impulse. This is because that loss is made up through the restriction at the exhaust, which controls the fill Mach number. Other research has shown that inlet Mach number is an important variable for detonation stability [47]. Generally, the higher the incoming flow Mach number, the greater the axial component of propagation becomes. It also changes the inclination angle of the detonation wave relative to the injection flow.

Rankin et al. [48] has shown results on how increasing the air gap of the RDEs baseline inlet changes the plenum response that is measured. Increasing the air gap size has multiple effects. It lowers the pressure drop across the inlet, which improves the ability to achieve an overall static pressure gain. However, it lowers the air stream exit velocity, which decreases the mixing effectiveness. While these two things are true, this work was still able to demonstrate up to a 15\% increase in static pressure across the inlet as air gap was increased. The downside to this change in the RDE is that the increased air gap opens up the inlet to a greater feedback effect from the detonation. The plenum measures higher pressure magnitudes the larger the gap becomes. This is what drives the need for designing a well-isolated inlet.

Significant work has been done in the past to better understand supersonic combustion systems, including RDEs. Since there has been little to no experimental work published on RDE systems with unique, novel inlet geometries, the need to improve and optimize the inlets remains unsolved. Some beginning steps have been taken in past work towards determining how to address superior inlet designs. However, none of this has been applied and accomplished in the way of studying novel RDE geometries. Optical access into the inlet plenums of the RDE is something that has not previously been reported on, and provides a valuable tool for analyzing the performance of the new designs tested in this work. 


\section{Chapter 3: Experimental Design}

To assist in the rapid testing of novel inlet concepts, a rig was designed and constructed to allow for quick, accurate, and simplified tests to be conducted on new inlet designs. The test rig was built as a housing for the inlet pieces to be placed in, and an outerbody to form the detonation channel. Incorporated into the housing were multiple methods for pulsating flow, measurement locations for pressure transducers, and points of optical access for visualization techniques. The device was designed as an unwrapped, scaled model of the RDE. It is a linear channel section that will simulate a slice of the RDE geometry. Essentially, the cylindrical geometry of the combustor is unrolled into a linear section, which is approximated by this device. The assembled device is shown in Figure 22, without any inlets inserted. The inlet sections will be 3 inch wide sectors of the RDE injection geometry. This is approximately $15-20 \%$ of the entire unrolled length (circumference) of the combustor. Through rapid prototyping, new fuel and air side inlet sections have been created to test in the device. It allows for simple and fast interchange of inlet concepts. By simply removing the bottom plate, and loosening one of the side plates, new geometries can be replaced quickly into the housing. The benefit of this housing is that it will allow for data collection that may not be easily achieved in the actual RDE, due to temperature and pressure constraints. Further details of this inlet testing platform are explained in [49]. Figure 21 shows the basis for the testing platform, and how it is derived from the full scale rotating detonation combustor (RDC).

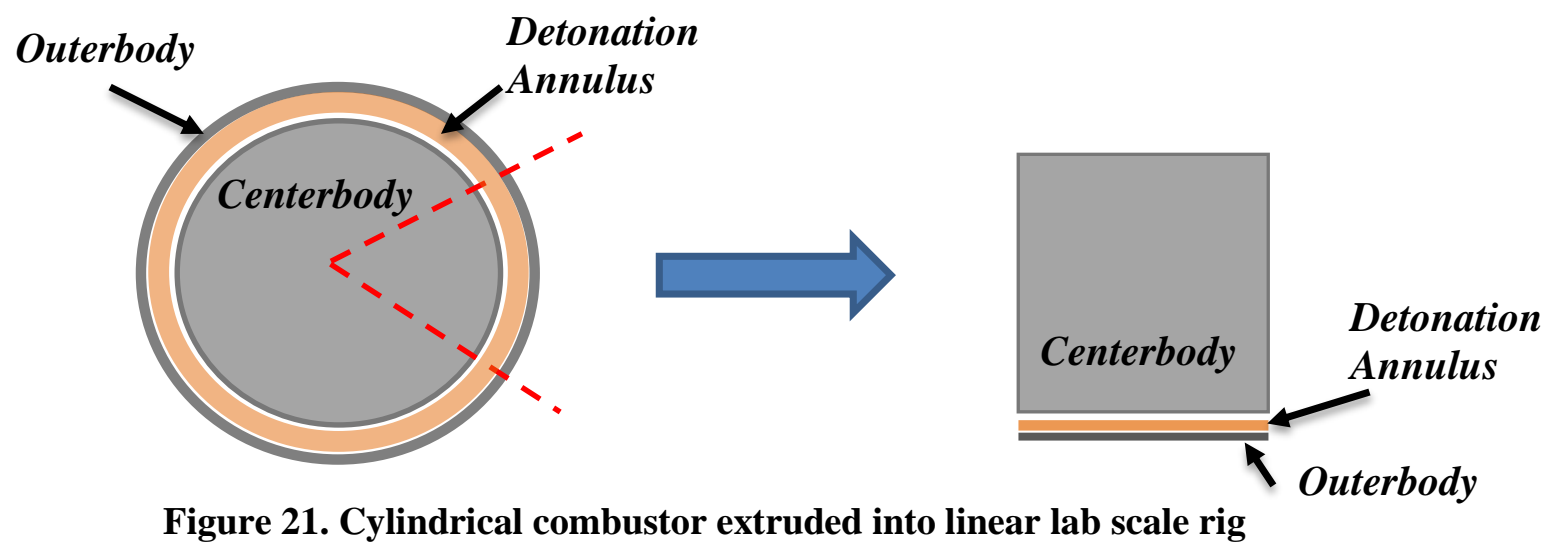

Figure 21. Cylindrical combustor extruded into linear lab scale rig 
Holes were machined into the top and bottom of the inlet sections to utilize dowel pins for proper alignment inside of their housing. The side and bottom openings were covered with quartz windows for transparent access through the side of the test section. The quartz provided good optical properties for visualizing small gradients in the flow. It has high transmission of light, in addition to a good refractive index that was ideal for imaging. Since quartz was used, it limited the operating pressures in the plenums of the tests to not more than 20-30 psig. Small rubber gaskets were used in the window slots to seal around each window. Sealant was also required on each side of the test section. This was achieved through small, custom made O-ring gaskets on each inlet's side walls.

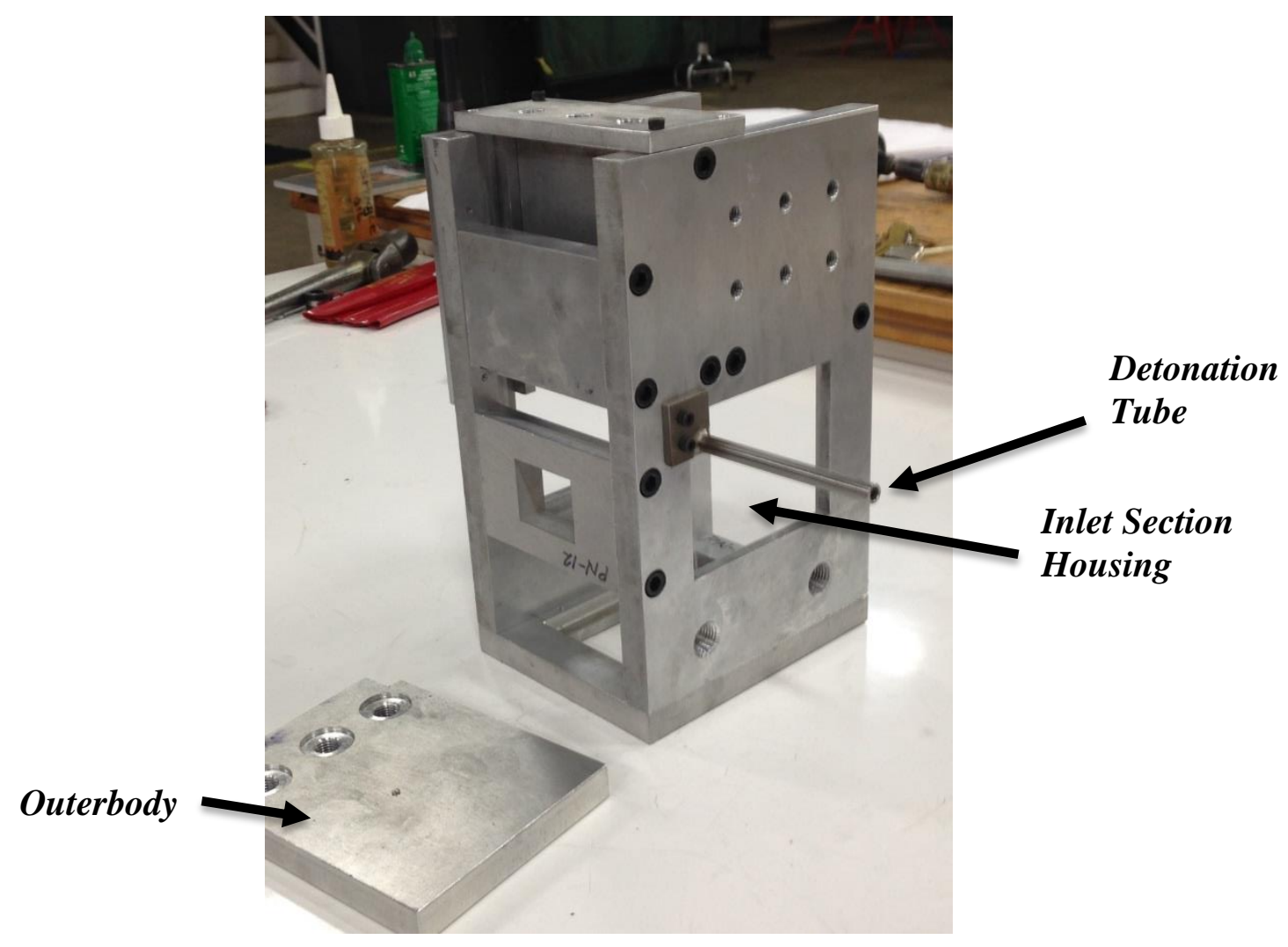

Figure 22. Housing for inlet testing with detonation tube mounted 


\subsection{Fabrication of Lab Infrastructure}

The $1 / 4$ " Swagelok tube on the outside of the device is where the flow enters the RDE channel. The primary use of this tube is a supply for a detonation, which is initiated upstream and allowed to travel through the tube and into the channel. The detonation tube is operated on hydrogen and air, which gives a comparable match to detonation cell size, since this is the fuel-oxidizer combination used in the hot fired testing at NETL. Generally, it is ideal to fire at an equivalence ratio at or near stoichiometric $(\varphi=1)$, since that is where optimal performance is typically achieved. However, for these experiments, the detonation tube was operated at a slightly richer $\varphi$. This was usually primarily at $\varphi=$ 1.2. The reason for firing slightly rich is most likely due to poor mixing in the tube. Since $1 / 4$ " tubing was used, it could also be an issue of heat loss to the tube walls. The surface area to volume ratio within the tube is larger for a smaller tube, so more volumetric heat loss occurs. By adjusting $\varphi$, there is limited, indirect control over the strength of the detonation source. An automotive spark plug, powered by a 5 volt spark ignitor, provides the initiation energy to ignite the detonation. Through the length of the tube, a deflagration-to-detonation transition is undergone so that by the time the wave reaches the inlet, a detonation is well established. By using two pressure transducers in the tube, the wave speed is measured to give confirmation of detonation. Knowing the separation distance of the transducers, as well as the time shift in the Von Neumann spikes recorded by each transducer, an accurate wave speed can be measured. As long as the wave speed is at or near the Chapman-Jouguet (CJ) speed, the detonation is validated. The pressure trace inside of the detonation tube from a simulation is shown in Figure 23, with the Von Neumann spike shown just before 0.025 milliseconds. This profile is similar to the pressure profiles seen from fired RDEs. Pressures measured in the tube also give a secondary confirmation to detonation. Where the tube transitions into the channel, the flow is highly diffused and drops significantly in pressure. Pressure measurements taken in the outerbody of the test rig provide pressures that are directly imposed to the inlet. These pressures are used for the scaling parameters. 


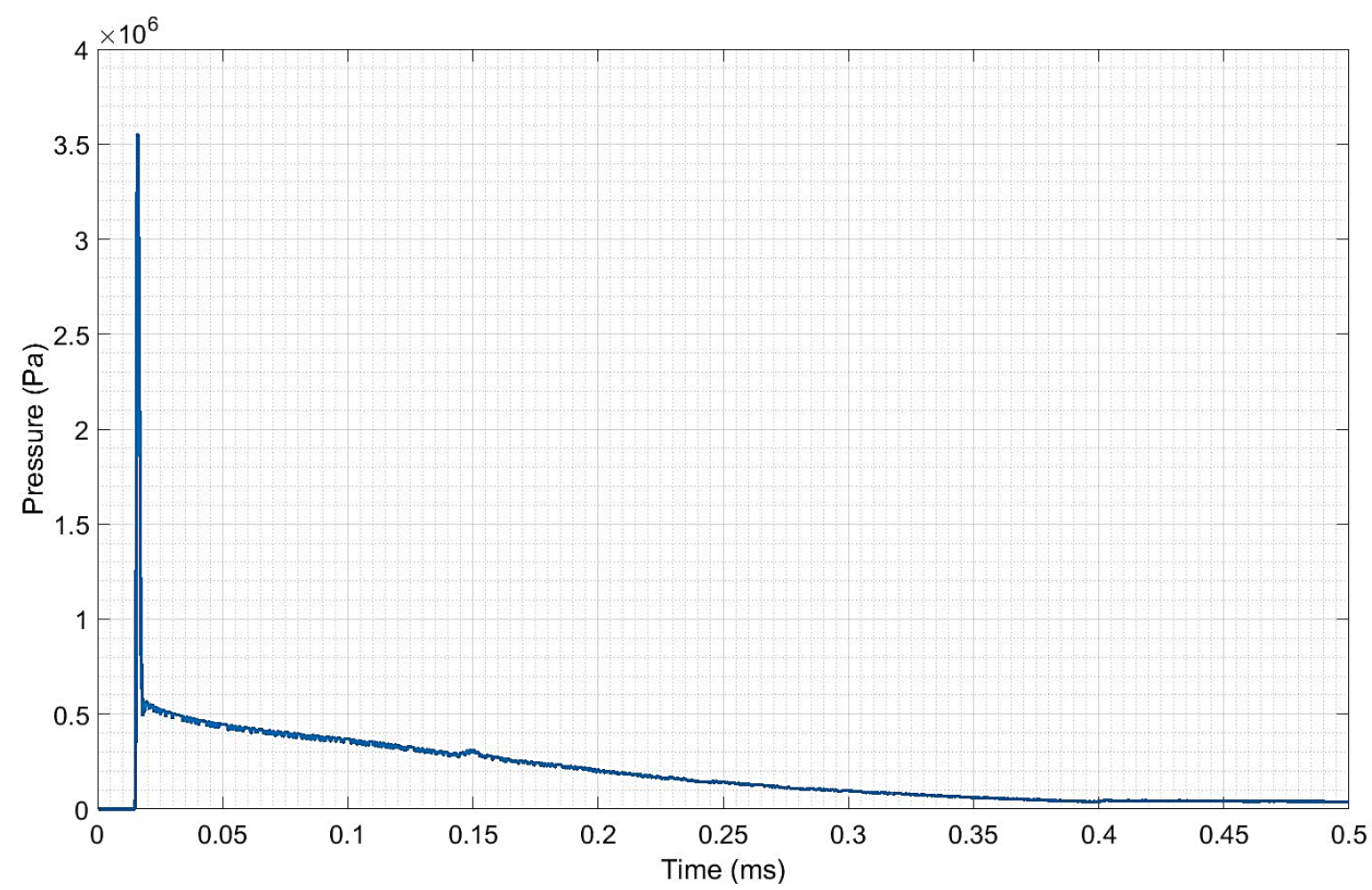

Figure 23. Pressure trace inside of detonation tube

This device was developed with the goal of matching critical non-dimensional flow parameters to the full-scale, fired RDE (FSRDE). The width of the combustion annulus in the FSRDE (0.2 inches) is equivalent to the channel width in the test rig. This enables a 1:1 geometric scale to be utilized and geometric similitude to be achieved. For the flow parameters, it was difficult to consistently match the Reynolds number of the scaled model to the full scale RDE, because the flow velocity in the FSRDE is high. It is possible to employ other scaling methods in the future, like Mach number scaling. Other flow characteristics, like turbulence intensity and turbulent length scales were unable to be matched due to the air flow rate limitations. However, the Strouhal number will provide an accurate method for assessing the acoustic or fluidic performance of inlet designs in terms of its frequency response. It will also responsibly scale the recovery times between the full scale and lab scale RDE (LSRDE), since it is a function of the convective time scale. The periodic nature of the detonation event inside of the 
combustor will be matched to the periodic forcing to the bench scale setup. The Strouhal number is defined in Equation 1.

$$
\begin{aligned}
& S t=\frac{f \cdot L}{u} \\
& t_{\text {conv }}=\frac{L}{u}
\end{aligned}
$$

In Equation 1, $f$ is the frequency of the detonations, $L$ is the characteristic length, and $u$ is the magnitude of the air flow velocity. The characteristic length is the size of the air gap, and since the geometric scaling is $1: 1$ between the lab testing platform and the full scale combustor, this length is matched. Therefore, the frequency is set based on the difference in air jet flow velocity between the LSRDE and FSRDE. The convective flow time scale, $t_{c o n v}$, shown by Equation 2, is contained within the Strouhal number expression. The flow rates capable of being provided in the lab-scale model are lower than those in the FSRDE. Therefore, reaching full dynamic similarity is not possible due to the large discrepancy in flow velocity that is possible in the bench scale setup. However, by scaling the pressure source to match the provided flow rates in the lab-scale setup, it can be matched well with what the FSRDE exhibits.

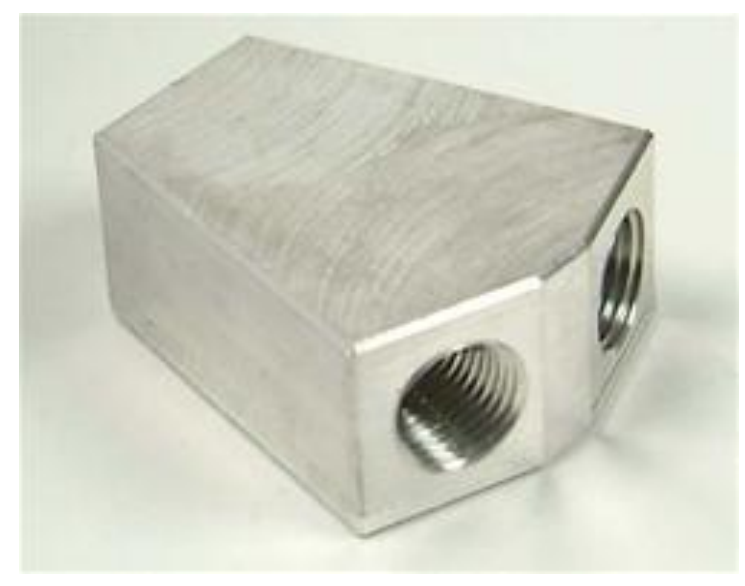

Figure 24. Flow splitter block 
The detonation tube is unable to fill and fire fast enough to produce multiple detonation shots in a short enough time interval. So while one shot from the detonation tube does not achieve pulsated flow delivered to the inlets, two shots can be achieved by splitting the single source of the detonation event. Using a splitter block, like the one shown in Figure 24, one inlet splits into two outlets. Assuming that the detonation evenly distributes into two ports, the pressures and wave speeds of the newly created detonation sources should be reasonably comparable. This enables the detonation source to be delivered via two separate tubes. By changing the length of these tubes, it will change the time that the detonation takes to reach the channel. Tuning the lengths of these tubes can achieve a wide range of desired frequencies by spacing the detonation wave arrivals. Figure 24 shows an example of the pressures measured from the two independent waves. The equivalent frequency of this configuration is around $2.8 \mathrm{kHz}$, very comparable to an RDE characteristic frequency. This frequency provided unique capability since the shockwave-to-shockwave interaction could be observed. While the shock trains are still present in the plenums, the new pseudo detonation passes through the plenum, allowing visualization to record how the two shock trains interact.

Two different setups were required to measure two different phenomena. The frequency of $2.8 \mathrm{kHz}$ enabled the shock-shock collisions to be observed. However, this does not scale well enough to match the Strouhal numbers between the FSRDE and the LSRDE. The frequency to appropriately match Strouhal numbers between the FSRDE and LSRDE is approximately 1,025 Hz. A sample data set from the split-tube configuration is shown in Figure 25, where there is a time shift between the pressure signals that corresponds to a frequency of $1,025 \mathrm{~Hz}$. 


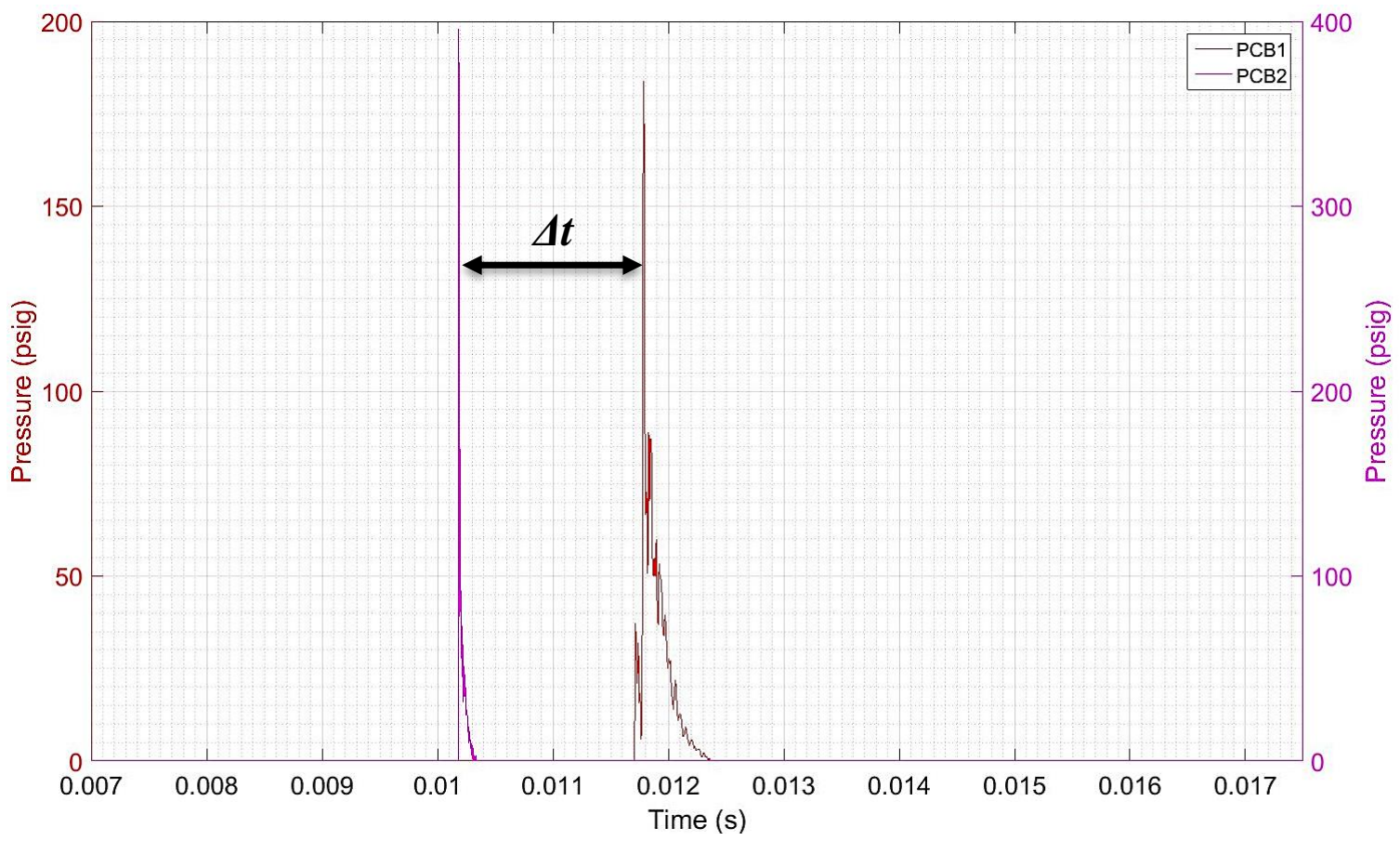

Figure 25. Pressure trace of detonation tube after splitter block

Since the main driving mechanism behind the backflow phenomena is an adverse pressure gradient, relating the pressures in the bench scale RDE to the full scale is crucial. The pressure coefficient, $C_{p}$, is an important dimensionless parameter that is utilized for scaling of the test rig [50]. It works well to relate the pressure amplitudes inside of the testing device to the pressures inside of the FSRDE. It uses the pressure drop across the inlet $(\Delta P)$ and the dynamic pressure in the detonation channel $(q)$ as scaling parameters. It is given by Equation 3 .

$$
C_{p}=\frac{P-P_{\infty}}{\frac{1}{2} \rho_{\infty} V_{\infty}^{2}}=\frac{\Delta P}{q}
$$


Using the pressure coefficient as a suitable scaling parameter is only achievable for the testing on the baseline inlet in the bench scale testing rig. Since there is no information about the pressure drop in the hot fired RDE with the novel inlet geometries in place, there is no way to gauge how to scale the pressure drop of the bench scale setup because there is no pressure drop information on that inlet in the full RDE. The other dimensionless number used to scale the benchtop device to the full RDE is the momentum flux ratio, $J$, which is often used for jet in crossflow scenarios. It is given by Equation 4, and contains the important pressure ratio of the static feed pressure $\left(p_{\text {jet }}\right)$ to the detonation pressure $\left(p_{\infty}\right)$. This also takes into account the air stream Mach number $\left(M_{j e t}\right)$, the detonation Mach number $\left(M_{\infty}\right)$, and the specific heat ratios for the air stream $\left(\gamma_{j e t}\right)$ and detonation region $\left(\gamma_{\infty}\right)$. The ratio of the detonation pressure $\left(p_{\infty}\right)$ to the inlet static feed pressure $\left(p_{j e t}\right)$ between the FSRDE and LSRDE is maintained as well, shown in Equation 11.

$$
\begin{gathered}
J=\frac{\rho_{j e t} u_{j e t}^{2}}{\rho_{\infty} u_{\infty}^{2}}=\frac{\gamma_{j e t} p_{j e t} M_{j e t}^{2}}{\gamma_{\infty} p_{\infty} M_{\infty}^{2}} \\
\left(\frac{p_{\infty}}{p_{j e t}}\right)_{\text {Full scale }}=\left(\frac{p_{\infty}}{p_{j e t}}\right)_{\text {Lab scale }}
\end{gathered}
$$

An extensive methodology for scaling the LSRDE to the FSRDE has been outlined. While it is challenging to scale and relate the experimental design to the full RDE, the important findings and conclusions will be drawn from comparison of each of the new inlet designs to one another. Valuable understanding of the novel inlet concepts will be drawn by evaluating their performance relative to one another. 
From simulations, the Mach number can be determined at any desired location. From experiments, the wave speed is also calculated, which reveals how fast the detonation front travels circumferentially around the combustor.

The speed of sound of the gases in the LSRDE and the FSRDE are targeted to closely match. For air injection, since air is used as the oxidizer for both setups, the speed of sound is matched well for the air plenum. This would of course change if oxygen enriched, or preheated air were used in the hot fired RDE. However, for the standard cases, the speed of sound in the air inlet plenum is matched for both devices. The fuel side inlet plenum presents more challenges though. Hydrogen is used as the primary fuel for NETL's RDE testing. The speed of sound in hydrogen is much faster than that of air, by almost four times as much. Since it is not within the design envelope to sustain or promote any detonation within the test section, hydrogen cannot be used on the fuel section of the LSRDE. Therefore, helium is chosen as a surrogate gas for hydrogen in the bench scale device. This allows the speed of sound to be more closely matched. Helium is a light gas, with a molecular weight much closer to hydrogen than standard air. The speed of sound, which is shown in Equation 12, is a function of the specific heat ratios $(\gamma)$, the specific gas constant $(\mathrm{R})$, and the static temperature $(\mathrm{T})$. The specific heat ratios for hydrogen and helium are 1.41 and 1.66, respectively, while the individual gas constants are 2,077 J/kg-K for helium and 4,124 J/kg-K for hydrogen. This makes the speed of sound for helium approximately 1.3 times the speed of sound for hydrogen, which is relatively comparable, and is better than can be achieved with most other gases.

$$
a=\sqrt{\gamma R T}
$$

The plenum pressure is limited by the maximum flow rate for air in this setup, which is $700 \mathrm{slpm}$, and the maximum fuel side flow rate is $140 \mathrm{slpm}$. Under this set of limitations, the following set of conditions were chosen to run testing. The run conditions 
were chosen to closely match those of realistic equivalence ratios that would be run in a fired RDE. The breakdown of the run conditions are outlined in Table 1. As air flow rate through the LSRDE increases, $\mathrm{H}_{2}$ detonation tube flow was increased as well. That is because as the air flow rate increased, the high velocity air exiting the injector scavenged the detonation tube mixture out. As a result, the local mass fraction of hydrogen was significantly impacted, causing detonations to be weaker and more inconsistent. To remedy the issue, the increase of hydrogen flow accompanied the increase in total air flow.

\begin{tabular}{|c|c|c|c|}
\hline \multirow{2}{*}{$\begin{array}{c}\text { Hydrogen Detonation } \\
\text { Tube Flow (slpm) }\end{array}$} & \multirow{2}{*}{$\begin{array}{c}\text { Air Flow Rate } \\
(\text { slpm })\end{array}$} & \multicolumn{2}{|c|}{ Helium Flow Rate (slpm) } \\
\cline { 3 - 4 } & 100 & 34 & 42 \\
\hline 1 & 300 & 101 & 126 \\
\hline 1 & 500 & 168 & 210 \\
\hline 3 & 700 & 235 & 294 \\
\hline 4 & & Effective $\varphi=0.8)$ & (Effective $\varphi=1)$ \\
\hline
\end{tabular}

Table 1. Operating conditions for LSRDE 


\subsection{Inlet Geometry Production}

The inlet components were printed using an Objet Eden ${ }^{\mathrm{TM}}$ 260VS 3D printer, manufactured by Stratasys ${ }^{\circledR}$. The printer allowed for the creation of complex geometrical features in a timely manner. It is capable of building layers with a resolution of 16 microns, and its maximum build size is 10 by 9.9 by 7.9 inches, which was sufficient for the size of the inlet components needed for testing in this setup. There are two options for post-processing components after printing. The printer uses two different types of material when creating the parts: support and build material. The build material is what gives the components their structure. The support material is what is used to fill voids and build upon. After printing is complete, the support material must be entirely cleaned off of the part. When prints were first being created, the main method employed for cleaning was a high pressure water jet that was housed in a tank. This required manual work to be done for cleaning. The second cleaning method used a holding tank with a sodium hydroxide solution that circulates and is heated. For support material that is water soluble, this allows the parts to simply be dropped in the tank so that the support material can get dissolved away. This is a more advantageous approach, since it reduces time, in addition to being a more simple way to remove support material from small passages or geometries. A transparent material (Vero Clear-RGD810), in addition to a white, hightemperature material (RGD525) are the two materials used in this work. They have sufficient material properties to be effective in this application. After many runs in the test device, the inlet sections showed no visible signs of deterioration or degradation from the detonation waves that contact the material. This confirmed the lack of need for unique after-treatments to the material (such as paint, surface coatings, etc.). Since the duration of the pressure pulses from the detonation waves are very short, there is no significant thermal effects induced to the inlet sections.

A LabVIEW control program that controls all of the flows and data acquisition was used for the testing. The LabVIEW program controls the detonation tube, the data acquisition (DAQ) system, and the camera triggering all in one program. The camera 
software is controlled and operated separately on its own computer. Upon firing of the detonation tube, the DAQ records data for a short amount of time $(10-50 \mathrm{~ms})$. The data is acquired at a sampling rate of $250 \mathrm{kHz}$. At a frame rate of 25,000 fps, the entire fuel and air plenums fit into the viewing window. The camera frame rate is typically set at $100,000 \mathrm{fps}$ to focus in on the ejection location. However, the frame rate is decreased for some of the designs to compromise between the frame rate and resolution to fit the entire section into the image. The firing of the detonation tube is synchronized with both the data acquisition and the camera recording, so that data can be analyzed side-by-side with frames from the images.

Sufficient instrumentation on the LSRDE allows pressures and wave speeds to be measured. PCB pressure transducers are placed in the fuel and air plenums, which measures how much of a response the plenums receive in proportion to the total pressures in the annulus. Pressure transducers on the outerbody measure the total mock detonation pressures, and can be used to find equivalent wave speeds as well. Ports 1, 2, and 3, designated in increasing order of detonation wave propagation, are shown in Figure 26. Static pressure transducers measure the static feed pressures in the chambers at the inlet ports for the air and helium sections. The injection ports for the air and helium are indicated by the blue and red regions, respectively.

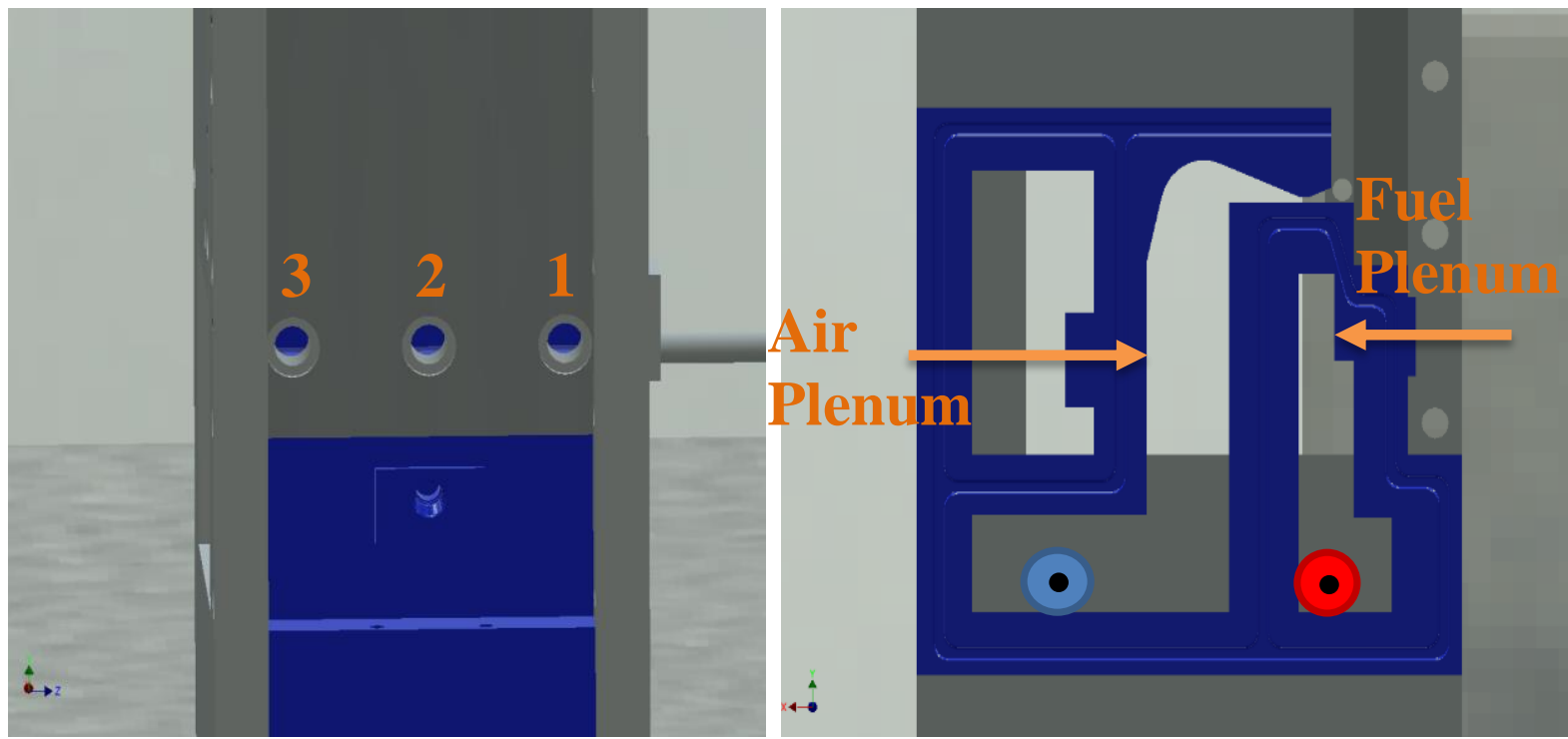

Figure 26. Instrumentation locations on LSRDE, outerbody ports on left, plena on right 


\subsection{Diagnostics Setup}

Visualization of the flow structures within the inlet is accomplished using a Schlieren technique. The first attempt at Schlieren imaging of the inlet was done using the conventional Schlieren method. This method consists of a broadband light source, and a set of lenses to direct the light through the test section, and onto the capture window. The schematic of this technique is shown in Figure 27. This method proved to be insufficient for the data that was desired. The quality of the images taken were unsatisfactory, lacking quality resolution. They appeared grainy, and especially performed poorly at resolving the shockwaves into their thin structures. Since there is no focus plane between lens 1 and 2, the Schlieren displays gradients that are present anywhere between the two lenses. Therefore, this Schlieren approach was aborted.

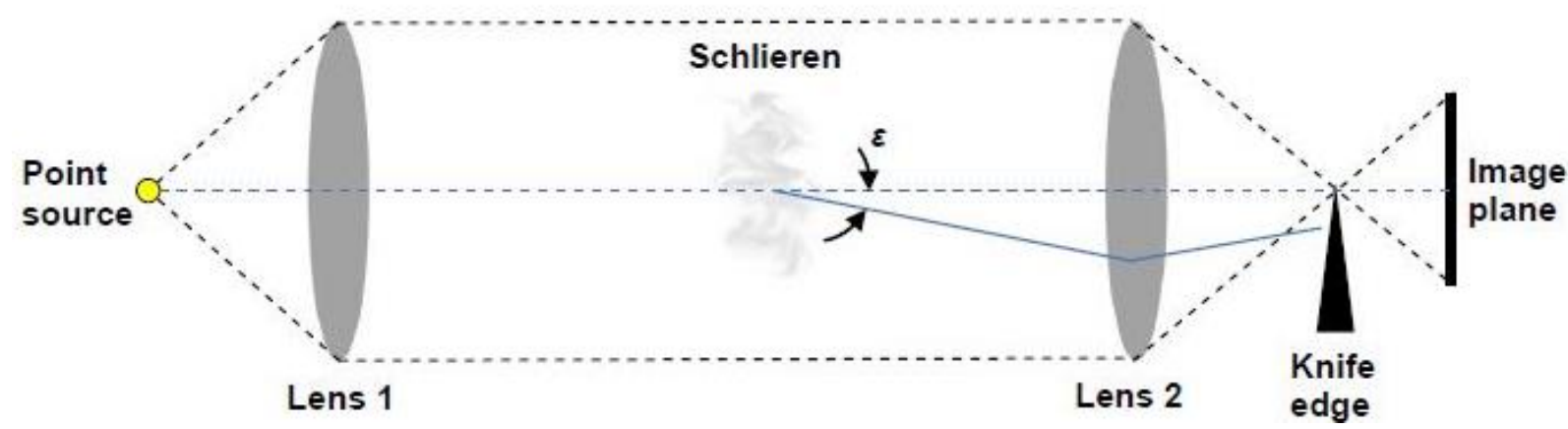

Figure 27. Conventional Schlieren technique [51]

The alternate imaging technique that was implemented was a focused Schlieren method. This differs from the conventional Schlieren in a few ways [51]. This method enables the focus of the viewing field to be narrowed down to a focal point, which can be moved to the desired location in the test section. A point source fiber light is used as the source of light. When using a spherical mirror just after the light, it can be fairly well collimated. By adjusting the camera lens' focus, the images taken in the test section are much sharper and more focused. A Phantom (C) V12.1 high-speed camera was used for recording the Schlieren video. A Nikon @ $200 \mathrm{~mm}$, f/4 AF-D macro lens was used on the 
camera to adjust focus and achieve the highest resolution for the rest of the Schlieren arrangement. An Olympus @ ILP-2 light source was used, which uses a mercury type UHP lamp. A layout of this Schlieren setup is shown in Figure 28. When the image is pared down to the desired viewing window of the test section, a resolution of $480 \times 400$ pixels is reached. At this given resolution, the camera is able to record at 25,000 fps. This is an even multiple number, which allowed for more convenient processing of the video frames. The frame rate was able to be increased up to 100,000 fps for higher temporal resolution of the flow features.

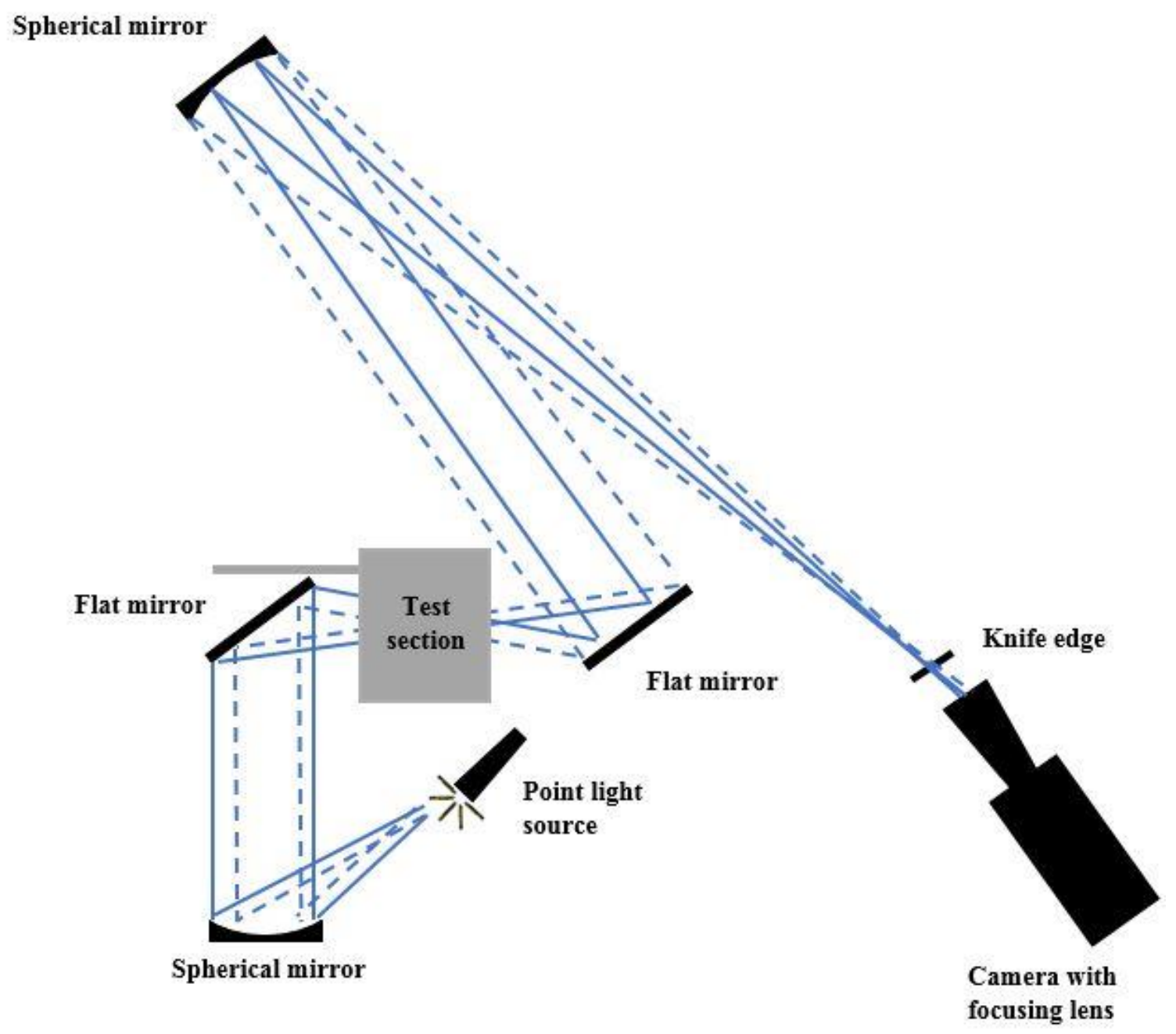

Figure 28. Optical arrangement for high-speed Schlieren diagnostics 
Enhancing the depth of field and resolution of the image in the test section required significant adjustment of the Schlieren setup. The ability of the Schlieren to focus in the test section was very sensitive to the focal length mirrors that were used. A shorter (12" FL) mirror was used temporarily, and proved to be inadequate for properly resolving the shockwaves. The shorter focal length exhibited characteristics more indicative of a shadowgraph technique rather than Schlieren. A double knife edge was used rather than one knife edge. This provided gradients in both the horizontal and vertical directions, which allowed for increased density gradient range to be captured [52]. 


\subsection{Simulation Validations}

CFD simulations were used as a method of testing preliminary design ideas and using their results as a guide for experimental studies. The CFD results were shown to be reliable in predicting many of the flow structures and behavioral tendencies of the RDE. Operational frequencies, peak pressures, and several other key combustion parameters compare well between simulation and experimental measurements. This method allows for a relatively quick way to gain significant understanding about a given inlet design, without the effort and cost of printing designs prior to understanding about their potential.

Using ANSYS Fluent, a full 3D, combustible model was developed for the RDE by Strakey et al. [53]. This model allowed for versatility and analysis of different inlet concepts. It also provided good insight to the propagation of flow upstream into the inlet manifold. By modeling a large portion of the inlet ducting, simulations produced information about the interaction of the flow with the manifold. A CFD model of the scaled RDE inlets was prepared, and was qualitatively compared to the FSRDE model to validate the efficacy of the experiments. The results compared very well with what was observed in the FSRDE simulations. While it is not presented in this work, the 3D CFD of the scaled inlet testing confirmed and validated the experimentally measured results.

For the sake of realizing parametric optimizations of the chosen inlet designs, 2D transient simulations were done on selected inlet geometries to enhance the geometry for maximum performance. The purpose of these simulations were similar to a parametric study, in which the geometry is tuned based on the simulation's output. This is a useful design tool, and helps discern the most appropriate details of the geometry to focus on. A pressure profile that is acquired from a 3D simulation of the detonation tube is imposed on the inlet face of the design as a pressure boundary condition. Mass flow boundary conditions are imposed at both the fuel and air inlets. Only the results from the simulations are presented here. The inlet designs themselves are discussed in more depth in Chapter 4: Inlet Designs Conceived. 
When the inlet geometry is exposed to pressure disturbances, fluctuations in velocity, mass flow rate, and pressure are observed. Flow disturbances from shock oscillations are observed in the CFD results. The interactions of shockwaves and acoustic waves is important towards understanding the regimes of flow that are present [54]. While the mesh is not nearly fine enough to resolve actual shock waves, the pressure profiles that result from the shock passing give information about how the pressure propagates through the chosen designs.

For the shock reflector design, which is presented in detail throughout the next chapter, it is important to know which of the two air streams should be selected as the 'primary' and 'secondary' air streams. The primary air stream, which is larger, allows a higher mass flow of throughput air, while the secondary air stream will possess a lower mass flow. It is important to know which air stream to deem primary and secondary for two reasons. Since the air flow is designed to recover before the fuel flow, it is necessary to determine how to appropriately size the streams of air to do just that. Design option 1 places the main air stream on the left of the fuel injectors. Design 2 places the main air stream on the right of the fuel injectors. Design 3 implements small cavities opposite of each of the air streams, which were intended to act as pressure relief volumes, so that rather than the pressure waves being forced through the streams of air, they could dissipate some of their energy into the cavities before pushing back through the air stream. These features did not appear to have any significant change on the ability of the design to reduce pressure feedback, or flow stream recovery. Figure 29 shows the three conceptual designs, as well as the location that surface monitors were setup to measure the main parameters of interest. The $x$-velocity component is monitored at the surface marked by the red line. The $y$-velocity component is monitored at the surface indicated by the black line. The plenum pressure component is monitored at the surface marked by the green line. The second reason that the sizing of the air streams is important is because it will change how the flows get interrupted, in addition to the dynamics of how pressure is propagated upstream. 


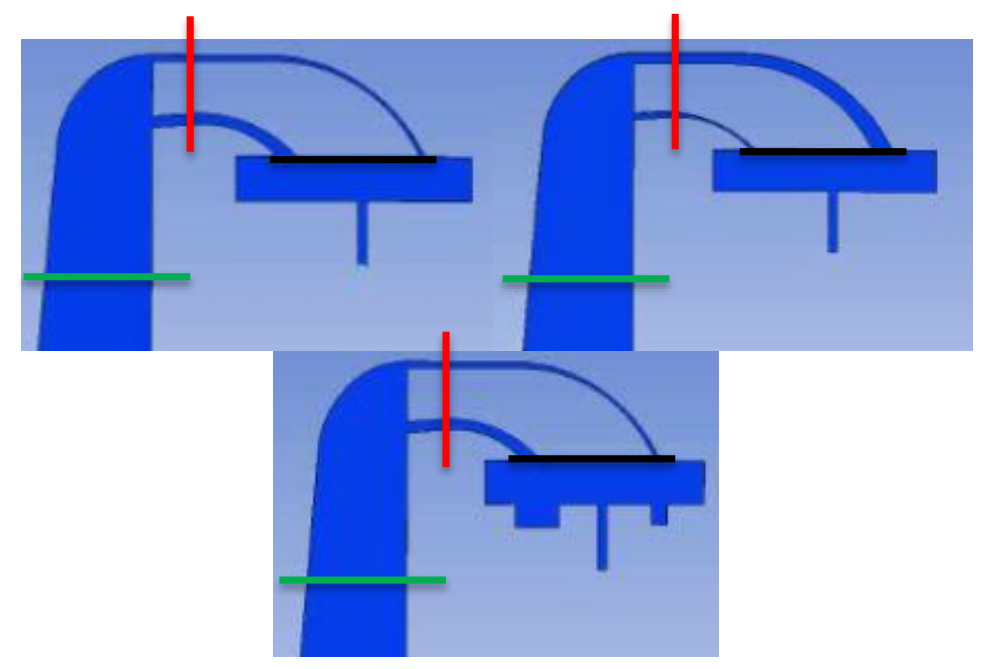

Figure 29. Reflector Design 1—top left, Design 2—-top right, Design 3—bottom

From the set of simulations performed for these two designs, it was observed that these three inlets performed at a very similar level. The performance of these three design variants is based on their ability to reduce peak pressures measured in the plenum, flow recovery ability, and its feedback resistance. A summary of the data observed from the simulations is shown in Figure 30. For instance, the pressure monitored in the air plenums was very close in magnitude for all designs. Therefore, the maximum plenum pressures were not notably impacted by the minor changes in any of the three designs. The species transport model allowed the combustion products to be measured throughout the simulation as well. Since the water vapor concentration profile is known for a detonation tube run, nitrogen is used to replace the water vapor and trace the backflow. The amount and rate at which species travel upstream through the inlet was revealing about the acoustic properties of that inlet. Design 2 outperformed its counterparts in terms of its potential to avoid flow disturbances in one of the air streams, which was confirmed by the mass fraction of Nitrogen $\left(\mathrm{N}_{2}\right)$ measured being less in both air streams. In fact, the secondary air stream for design 2 reported negligible species transport. The air velocity changes directions and recovers quicker for design 2, which is clear from sharper gradients of the velocity plots. By making the left air stream the secondary (smaller duct size), it is only subtly affected by the detonation events. This is clear in the mass fraction plot, as well as the plots of $\mathrm{x}$ and $\mathrm{y}$-velocity. 

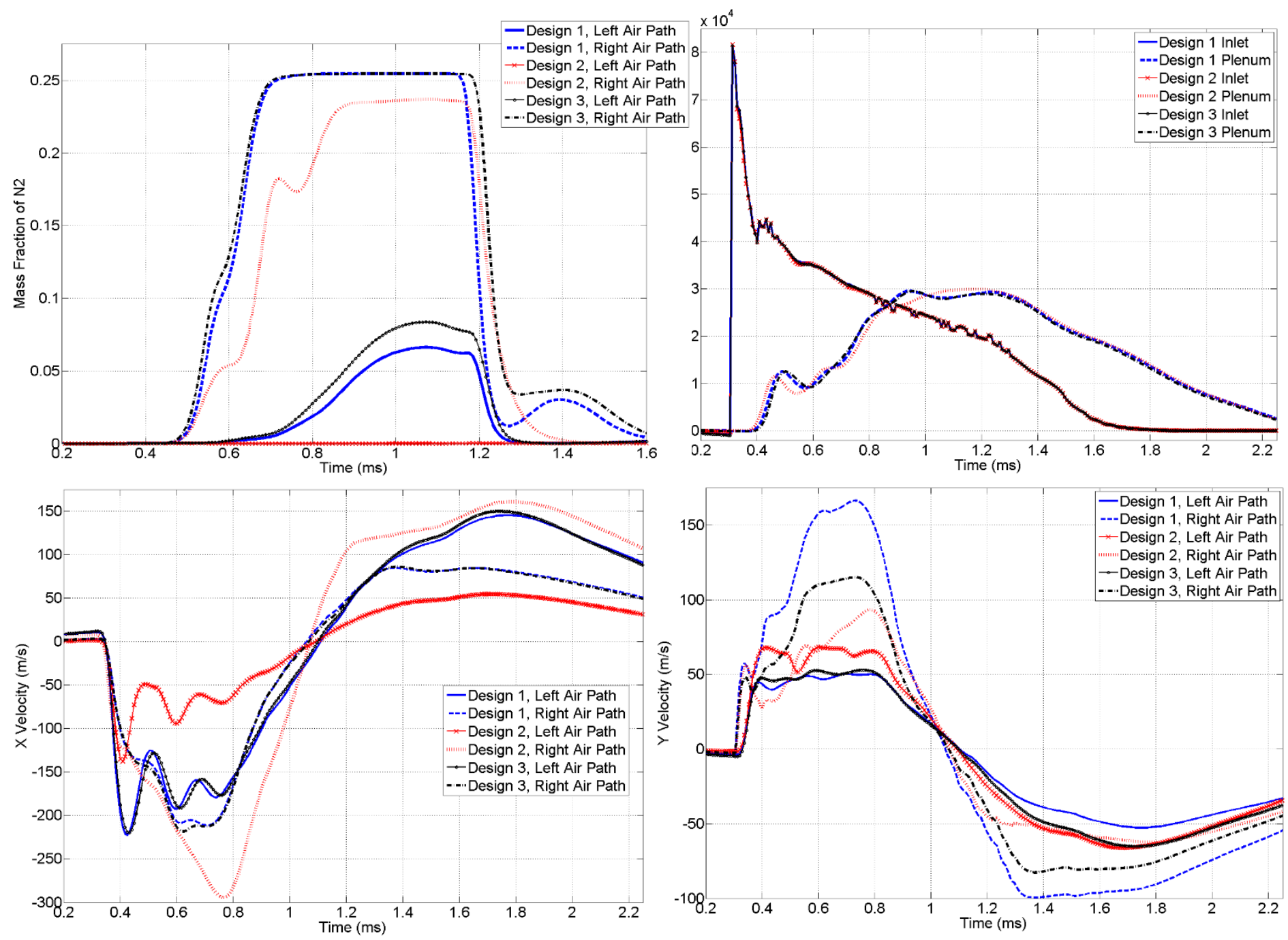

Figure 30. Simulation results for reflector designs for mass fraction, pressure, $x \& y$-velocity

Steady, 3D, compressible flow simulations were done for the wedge inlet geometry to provide a first order approximation towards its mixing potential. Since the wedge inlet is mainly the only novel design that introduces a significant change to the flow pattern of the injection, it is necessary to understand at some level how that will affect the interaction of the fuel and air streams. Since the experimental setup employed for this study did not provide any means for measuring mixing or air-fuel-jet interaction, the simulation can give some data towards that. It also provides some knowledge 
regarding the change in injection orientation, relating to the swirl and injection angles. The wedge injection geometry introduces a new component of velocity to the inlet flow. The coordinate system describing the RDE in this context is shown in Figure 31. Rather than predominantly being injected radially, the inlet flow due to wedge injection has a component in the radial direction and the azimuthal direction. A comparison is drawn between the wedge injector and the baseline injection scheme as a point of reference. The uniformity index, which is a statistical measure of the gas flow distribution across a face, is used to determine the spreading of the fuel across the plane of the annulus.

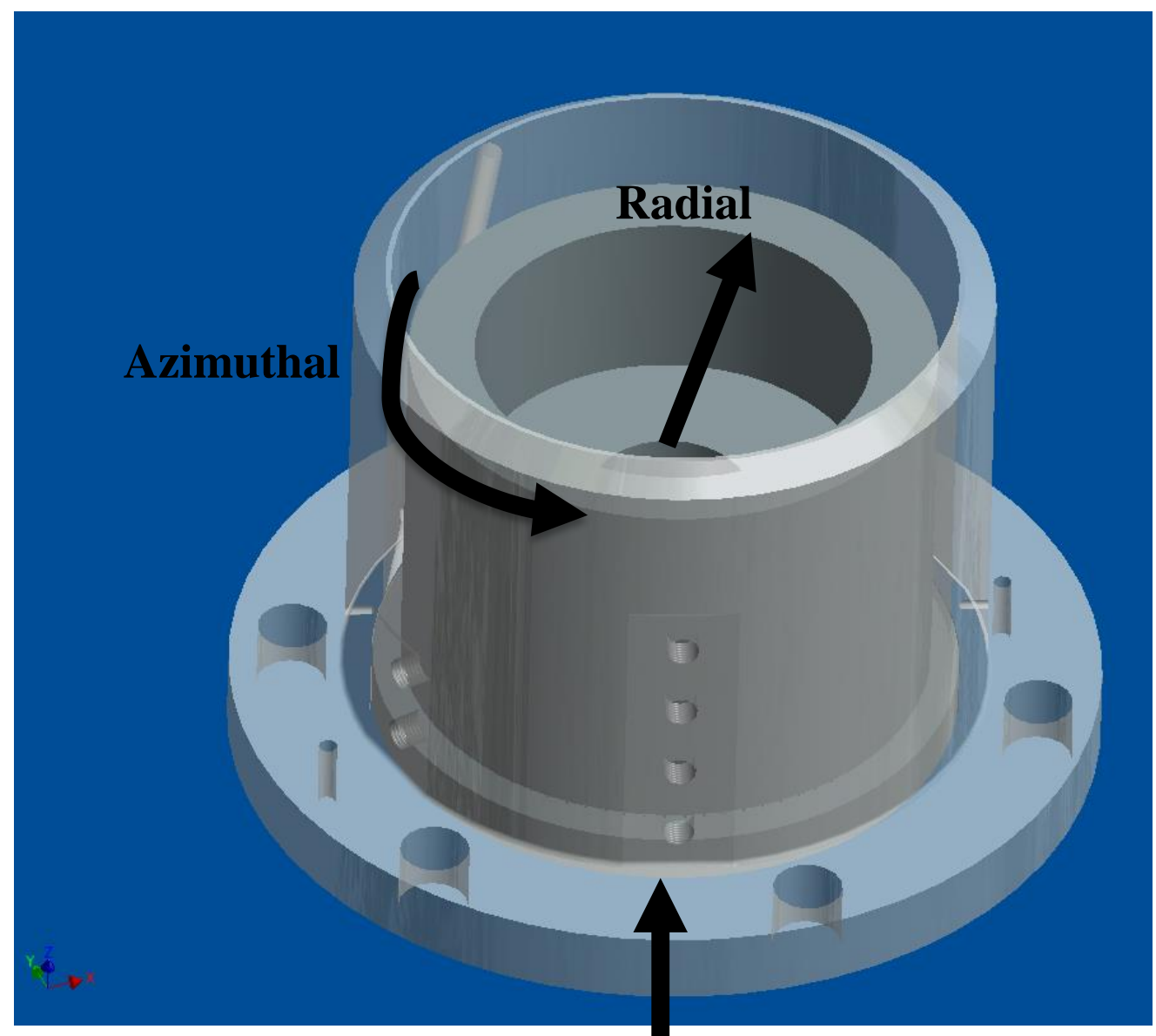

Axial

Figure 31. Directions of reference in the RDE

The wedge inlet was compared to the baseline injection geometry to give a reasonable method for comparison. The mixing data was taken at the injection plane, 
which is at the outer surface of the centerbody. One difference between the wedge inlet and the baseline is that the wedge inlet introduces the fuel into the air stream in the detonation annulus. However, the baseline inlet injects fuel at the divergent portion of the nozzle, which is slightly before it dumps into the annulus.

The baseline inlet provides poor azimuthal spreading of the fuel into the air stream. Column shaped structures formed by the fuel jets are clear in Figure 32. The uniformity index of the mass fraction of hydrogen is a good indicator to the mixing quality in the channel. The wedge injector resulted in a uniformity index of 0.55. The uniformity index gives a surface averaged value across a given plane. Compared to the baseline's uniformity index of 0.5125 that is a $6.8 \%$ increase in the mixing quality at the plane of injection. The difference in mixing quality of the two inlets is obvious in the figure. The wedge design exhibits significantly more lateral spreading of the fuel streams.

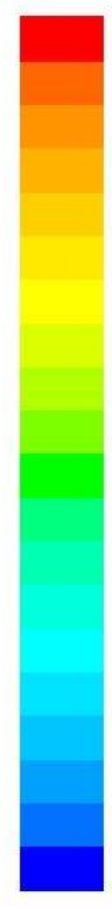
$1.00 e^{+00}$
$9.50 \mathrm{e}-01$
$9.00 \mathrm{e}-01$
$8.50 \mathrm{e}-01$
8.00e-01
7.50e-01
7.00e-01
6.50e-01
6.00e-01
5.50e-01
5.00e-01
4.50e-01
4.00e-01
3.50e-01
3.00e-01
2.50e-01
2.00e-01
1.50e-01
$1.00 \mathrm{e}-01$
5.00e-02
$0.00 e+00$
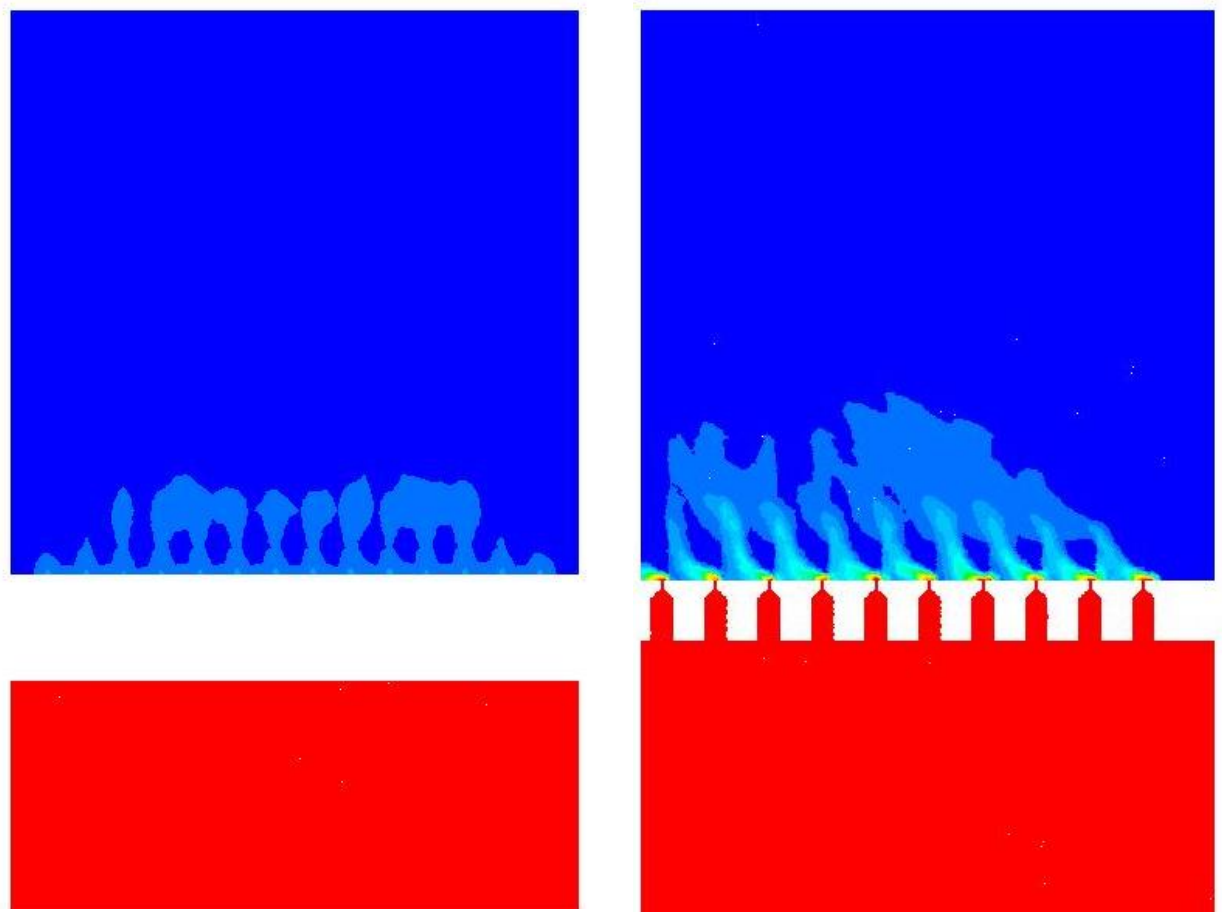

Figure 32. Hydrogen mass fraction contours at injection plane—-baseline on left, wedge design on right 
Another important characteristic of the wedge injector is the increase in fluid momentum in the circumferential (azimuthal) direction. The detonation wave travels circumferentially during RDE operation. The bulk of the fluid velocity for the baseline inlet travels in the axial direction. It can be seen from Figure 33 that there is virtually no $\mathrm{x}$-direction (azimuthal) velocity for the baseline inlet design. Therefore, the detonation wave has to push through the mixture perpendicularly. The wedge injector adds a dominating component of velocity that is in the circumferential direction. This should contribute to the energy potential of the detonation wave.
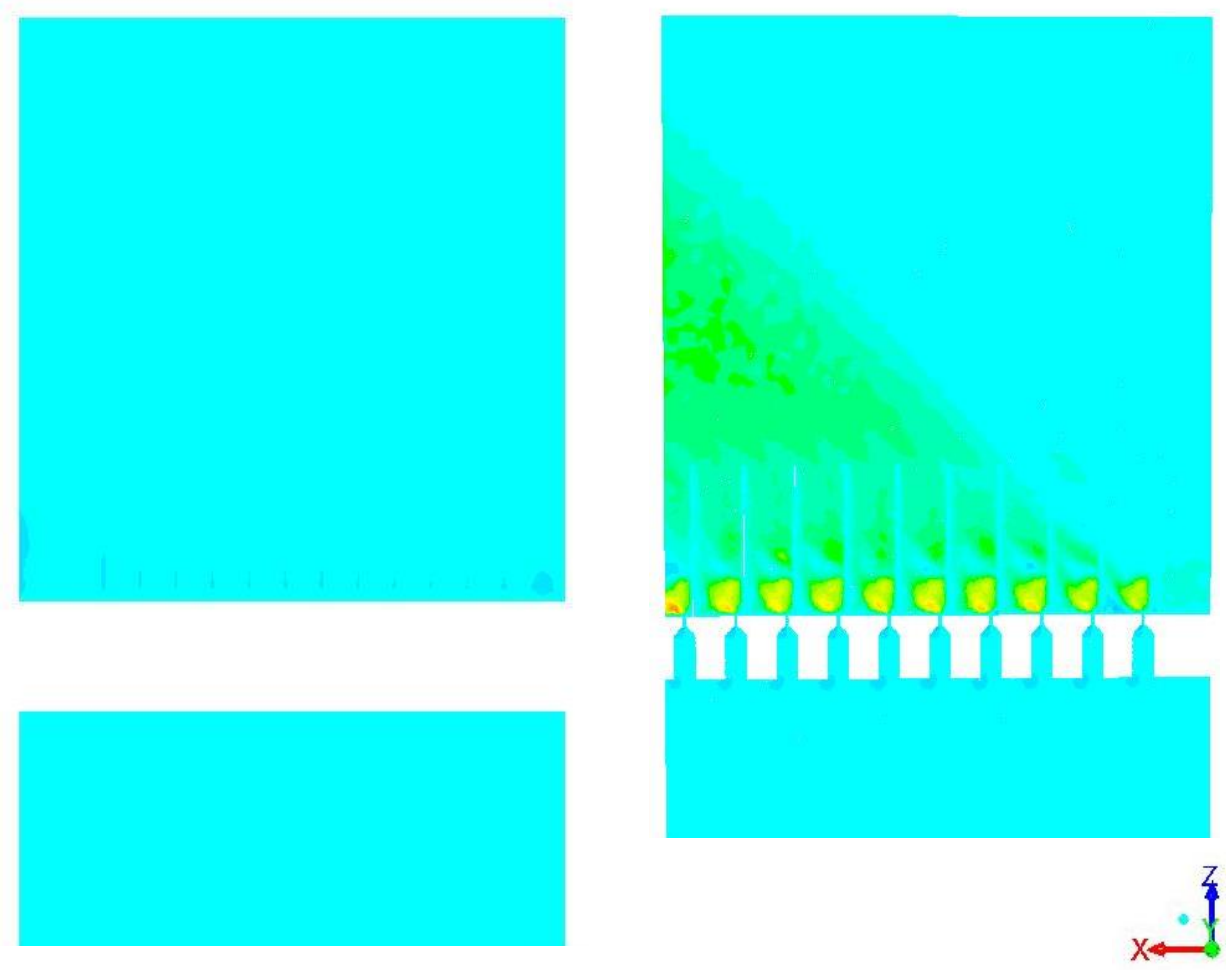

Figure 33. X-Velocity contours-baseline on left, wedge design on right 


\section{Chapter 4: Inlet Designs Conceived}

The various methods of fuel and air injection are discussed in the following section. With each novel inlet design considered, a brief explanation regarding the approach and intent behind its design is presented. The RDE operates at a characteristic frequency, which is the number of times the detonation wave makes a full cycle in one second. The target was to design a geometry with acoustic properties such that the key harmonic frequencies of the RDE will either be damped out by the inlet, or reflected back into the detonation annulus.

If the resultant shockwave from the detonative combustion can be leveraged for compression of the inlet air, the energy extracted from the inlet flow can be maximized. This will generate a supercharger effect, as the pressure waves compress the air ahead of it. This phenomena occurs when pressure waves produced as a result of combustion are used to compress the intake air. Another advantage of the shockwaves in the inlet are the low pressure regions that trail behind a shockwave. These lower pressure regions aft of a pressure wave can be used to pull in fresh mixture from the inlet behind it. This is what is known as the Kadenacy effect [55]. The Kadenacy effect is the main driving mechanism for pulse jet engines, and can be a very effective engine cycling principle. The Kadenacy effect can also be implemented by tuning of a Helmholtz resonator [56]. For these reasons, it is desirable to reflect and redirect the shockwaves back downstream towards the combustor. It is tempting to discover ways of dissipating the shocks in the inlet plenum. Rather than damping out the energy from shockwaves, which is usable for potential work of the flow, it is more efficient to extract the most work from them by reflecting them away from the upstream inlet.

It is also believed that the shockwaves can be reflected and used to reinject into the combustion annulus. This will serve several purposes. It will assist in preventing the backflow of products into the inlet. The high pressure energy provided by the shocks will 
work against the feedback that is trying to be prevented. This will also help recover some of the static pressure loss in the system.

The first set of tests are done for the same baseline inlet geometry with varying air gaps. All of the hot fired RDE tests done at NETL up to this point have been with an air gap of 0.022 inches. Three new air slots are tested: $0.022,0.044$, and 0.066 inches. These correspond with air gaps that have been experimentally tested at AFRL. While there is quantitative data that was reported on the plenum dynamics at these cases, there is no flow visualization to supplement these tests.

The cross sectional areas of the throat of each inlet is maintained relatively consistent. This maintains the comparability of the inlet pressure as a function of flow rate. This also ensures that the detonation has approximately the same area to push back through the inlet. 


\subsection{Wedge Inlet Design}

The first proposed inlet design concept is one in which wedges are present in the combustion annulus, such that the direction of the detonation propagation advances "up" each ramp. The air is injected from the backside of the ramp, in the leg of the right triangle that forms the wedge. Concerns with this injection scheme are the stability of the detonation wave, in addition to the complex and strong shockwave reflections and interactions off of each ramp. This concept is adapted from other applications in which a ramp style geometry is used as a means for fuel or oxidizer injection [57]. This style of injectors will induce significant swirl in the inlet air stream, since the air is introduced at an increased tangential angle. In fact, the design is essentially a radial swirler for the combustor. However, it has been observed that increasing swirl in the inlet flow stream may increase the stability of the detonation. It was also believed that recirculation zones would be formed aft of the ramps, which would be instrumental in the mixing of the fuel and air streams. The wedges were designed also so that it generates more turbulence through the detonation channel, ensuring that the combustion remains in the detonation regime. In this sense, it will act akin to a deflagration-to-detonation transition (DDT) device [58], which helps to stabilize the flame front in detonation mode. The wedges will also induce shock reflections from its surface, also acting to enhance DDT [59]. DDT devices are generally recommended to be avoided, because they can cause decrements to the system's performance. While no combustion is sustained in the annulus for this study, there is no way of confirming the true effect the area irregularities that the wedges will create. Concerns about detonation extinction, reignition, and other changes to the detonation due to the area changes should be further confirmed and investigated [60]. However, this is a first step at realizing isolation of the inlet from the detonation annulus. Once the first hurdle is overcome, the next challenges will be addressed in their appropriate order.

The wedges must be constructed so that the maximum and minimum channel width still falls within the tolerance for the cell size of the given fuel and oxidizer 
combination. For hydrogen and air detonations, the cell size, $\lambda$, is estimated to be around $8 \mathrm{~mm}$. The cell size is the foundational structure of a detonation, and without satisfying this minimum cell size, detonation will not be established. It has been shown that as long as area changes are sized appropriately, they can still be conducive to sustaining detonations [62]. The critical fill height, $h^{*}$, is a function of cell size only, which is shown in the Equation 12. The critical fill height is the required height of the fresh reactants necessary to sustain continuous detonation. A relation for the minimum channel width, $\Delta$, was established so that it obeys Equation 13.

$$
\begin{gathered}
h^{*} \sim(12 \pm 5) \lambda \\
\Delta \sim 0.2 h^{*}
\end{gathered}
$$

In these studies, this minimum and maximum channel width are not critical, since there is not a detonation that must be sustained. However, in extrapolating this design to the full RDE, this channel width must be carefully considered according to these empirical relations. Modifications of the wedge angle and the resultant wedge thickness must adhere to these limitations on the detonation cell size constraint. There is ample room for optimization of this design. The wedge design presented was a first attempt at identifying the wedge design as a possible inlet candidate. Through parametric studies and other means, the wedge angle, leg height, leg length, and other features of the wedges can be refined to be more suited for exposure to detonations. 


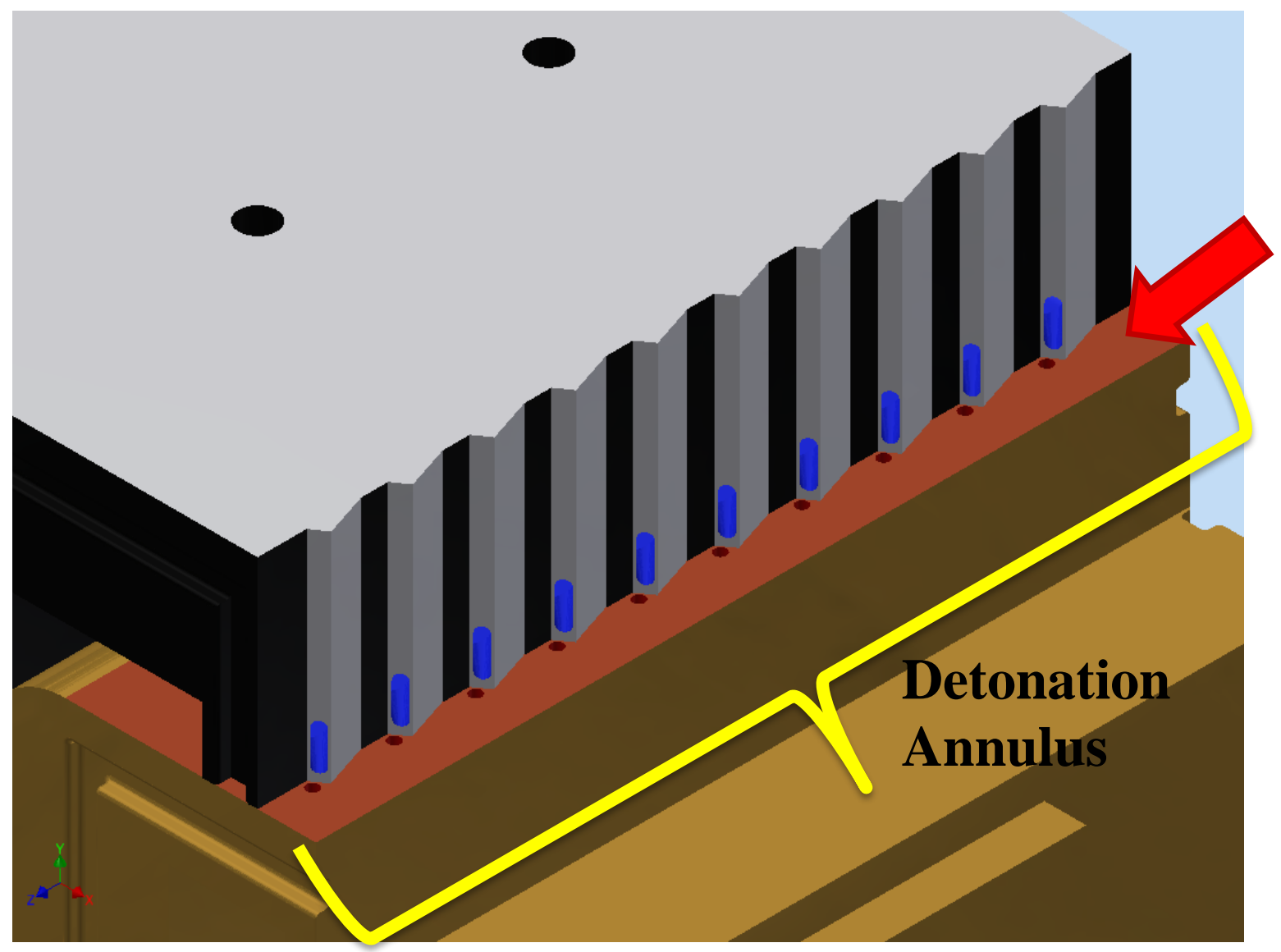

Figure 34. Wedge inlet design

The direction of intended wave propagation is indicated by the arrow in Figure 34. This design would be non-ideal in the case of a wave directionality shift, since the direction of the wedges would be facing in the opposite direction relative to the rotating direction of the detonation wave. However, wave directionality and stability is an entire focus area of research for RDEs, and is considered outside the target of this study. It is important to point out that wave stability and mode switching is highly dependent on mixing, flow rates, and chamber pressures [61]. Therefore, if mixing is improved as a result of this design, the wave mode switching problem may solve itself. Although these wedges may provide more stability to the detonation, which would decrease the likelihood of wave direction shifting. The ramp edge and shock wave interaction have 
been found to enhance supersonic flame stabilization [63]. There are fewer number of fuel injectors on this geometry compared to the baseline geometry, because the fuel injectors were strategically placed in relation to the wedges. Since 10 fuel injectors are present in this design, compared to the 14 injectors on the baseline design, the diameter of the fuel injectors on this design were increased to match the total cross sectional area of the fuel injection plane. 


\subsection{Busemann Biplane Inlet}

In an attempt to cancel the shockwaves traveling through the inlet, a configuration of opposing symmetric wedges is used on the inlet. This creates a type I shock interface [64], in which the intersection of two shockwaves are used to have a cancellation effect. This type of design was first suggested by Adolf Busemann, but for a much different application. The basic design is shown in Figure 35. The application of his theory was initially recommended for wing profiles on supersonic aircraft, to reduce both wave and pressure drag. However, for shockwaves traveling through the inlet of a RDE, this geometry could serve well in dissipating shockwaves. The 'favorable interference' of the geometry could be enough to reduce the loss effects of the shockwaves [65]. The most practical placement of this design is on the air side inlet, since there is more available space. The critical dimension for this geometry is the ratio of the area of the inlet, $A_{i}$, to the area of the throat, $A_{t}$. The ratio is determined by the design freestream Mach number, $M_{\infty}$, along with $\gamma$, the ratio of specific heats, as shown in the Equation 14 . The freestream Mach number in this situation is the detonation Mach number.
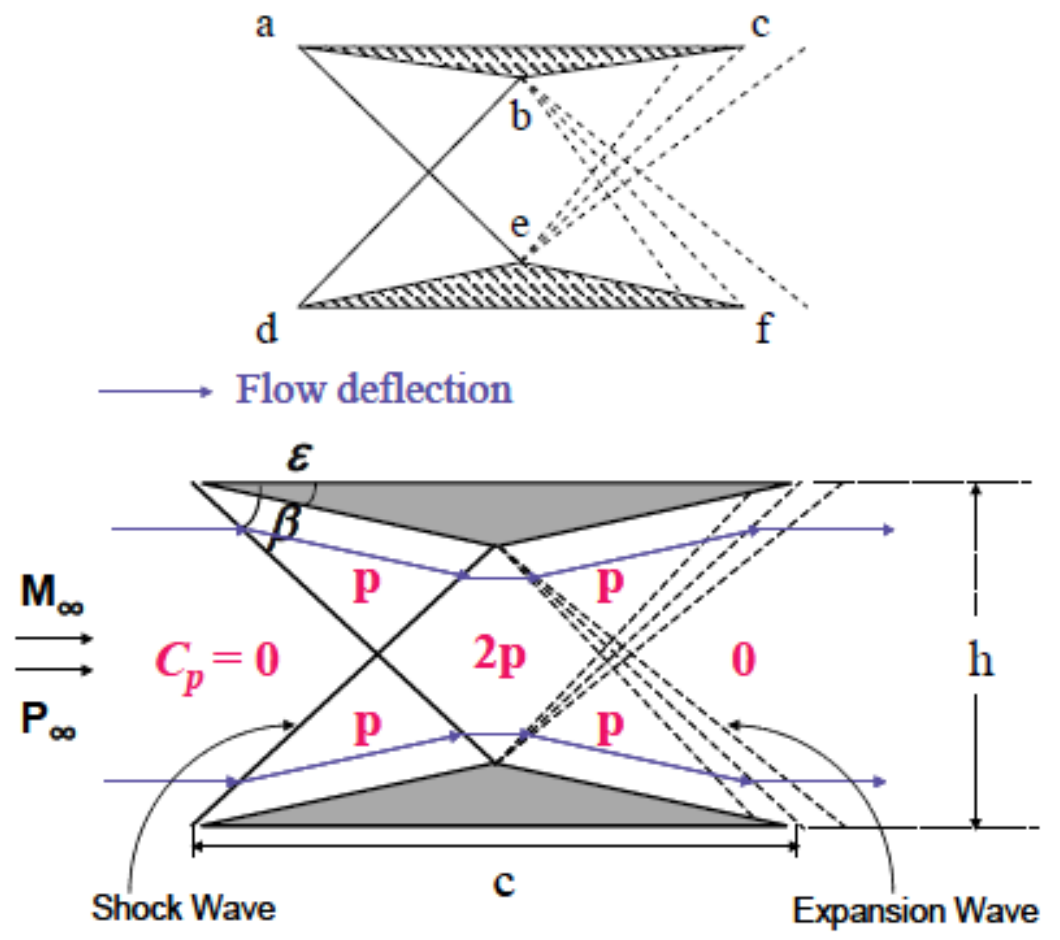

Figure 35. Portrayal of Busemann opposing wedge theory [66] 


$$
\frac{A_{t}}{A_{i}}=\left[\frac{(\gamma-1) M_{\infty}^{2}+2}{(\gamma+1) M_{\infty}^{2}}\right]^{\frac{1}{2}}\left[\frac{2 \gamma M_{\infty}^{2}-(\gamma-1)}{(\gamma+1) M_{\infty}^{2}}\right]^{\frac{1}{(\gamma-1)}}
$$

Several other geometric parameters of the Busemann geometry are evaluated. The wedge angle and thickness-chord ratio are two of them. Since these two features are less critical, and not dependent on Mach number, they are not focused on as heavily. However, they are kept close to suggested values in literature. The wedge angle, $\varepsilon$, is approximately $5.7^{\circ}$, and the $\mathrm{t} / \mathrm{c}$ value is 0.05 . This start limit for the Busemann design is shown to be strongly dependent on inlet Mach number [67]. Since Mach number is not accurately known throughout the inlet, it is estimated based on Schlieren images. It is assumed that one passing of the detonation wave does not affect the gas temperature drastically enough to significantly change Mach number. While static temperature drives the speed of sound, it is difficult to analytically predict the static gas temperature as a function of time in the inlet plenum. It is possible to acquire this value from high-fidelity simulations in the future. It was also desired to remain as non-intrusive to the flow as possible. High-speed heat flux gages may be incorporated in the future to better measure temperature in the plenum as a function of time (or wave passes). When the shockwave is pushed back through the throat of the inlet, the area contraction has several key effects on the shock. Peak pressure, shockwave strength and speed are all significantly reduced when traveling through an area contraction [68]. Magnetohydrodynamics (MHD) could be especially useful in conjunction with the Busemann design.

To maximize its potential effectiveness, two wedges are used in succession of each other in the printed design. The wedges are employed in the throat of the inlet. The average Mach number through the plenum was calculated from Schlieren images. By manually tracking the shockwaves through the plenum, knowing the distance they travel and the time difference between each frame, the shock front velocity is found. While the exact temperature in the plenums is not well known, it is assumed that it is not significantly affected by the single passage of the detonation wave. Therefore, the throat- 
inlet-area ratio is slightly larger than 0.9 to accommodate for a Mach number of approximately 1.3-1.4. The adapted concept of the Busemann geometry is illustrated in Figure 36, along with an exaggerated detail view of the wedge shape.

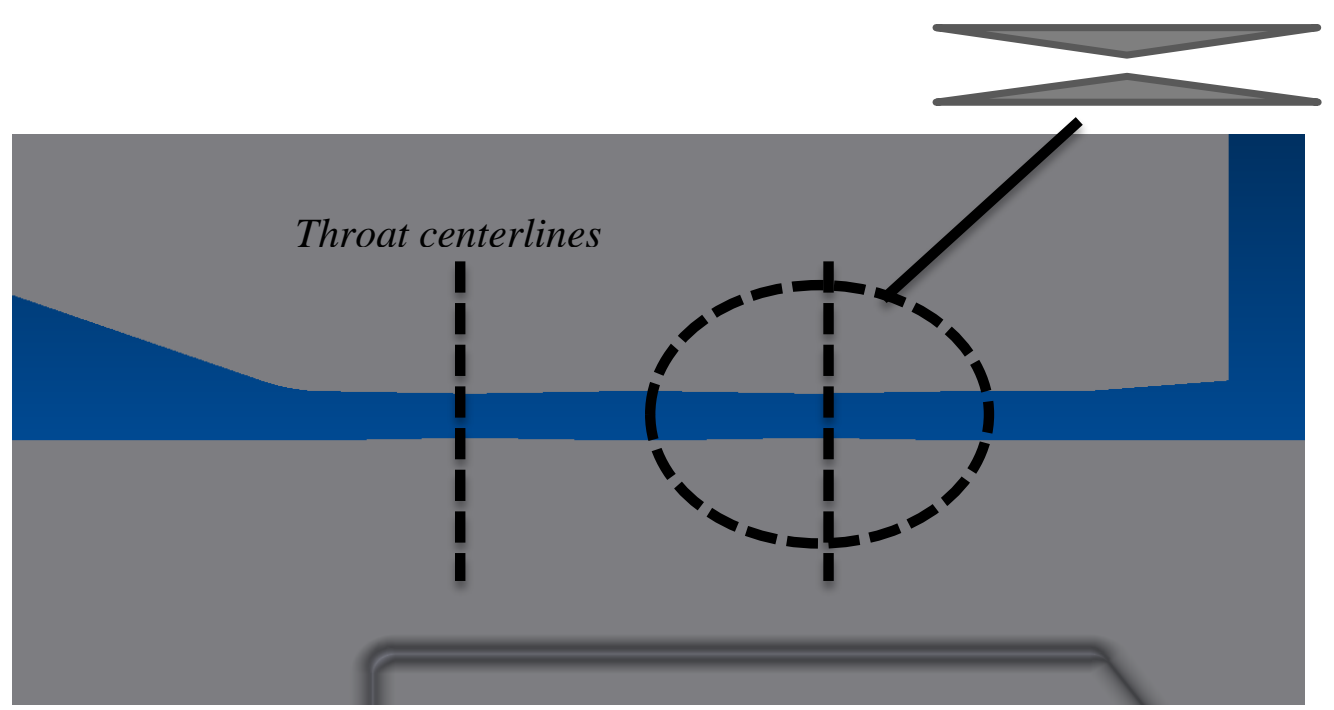

Figure 36. Design rendering of Busemann inlet (throat of nozzle) 


\subsection{Spade Fuel Diode}

Modifications to the fuel injectors were also examined. Since fuel plenum pressures and flow rates are much less than those of the air plenum, the fuel injectors are more susceptible to feedback from the detonation channel. Therefore, if the individual injectors can be better defended against this feedback, the disturbance to its flow can be minimized. The first geometry change to the fuel injectors that was conceived was coined as the 'spade'. It is called that for its resemblance to the spade shape seen on traditional playing cards. A CAD sectioned view of it is shown below in Figure 37, beside the traditional spade. This spade geometry is 360 degrees continuous around each fuel injector. Its design is such that the forward flow (indicated by the green arrow), has an unrestricted flow path, while the reverse flow (indicated by the red arrows), has an arduous path such that the flow is forced to change direction. This causes a lot of dissipation and retardant effects on the backflow.
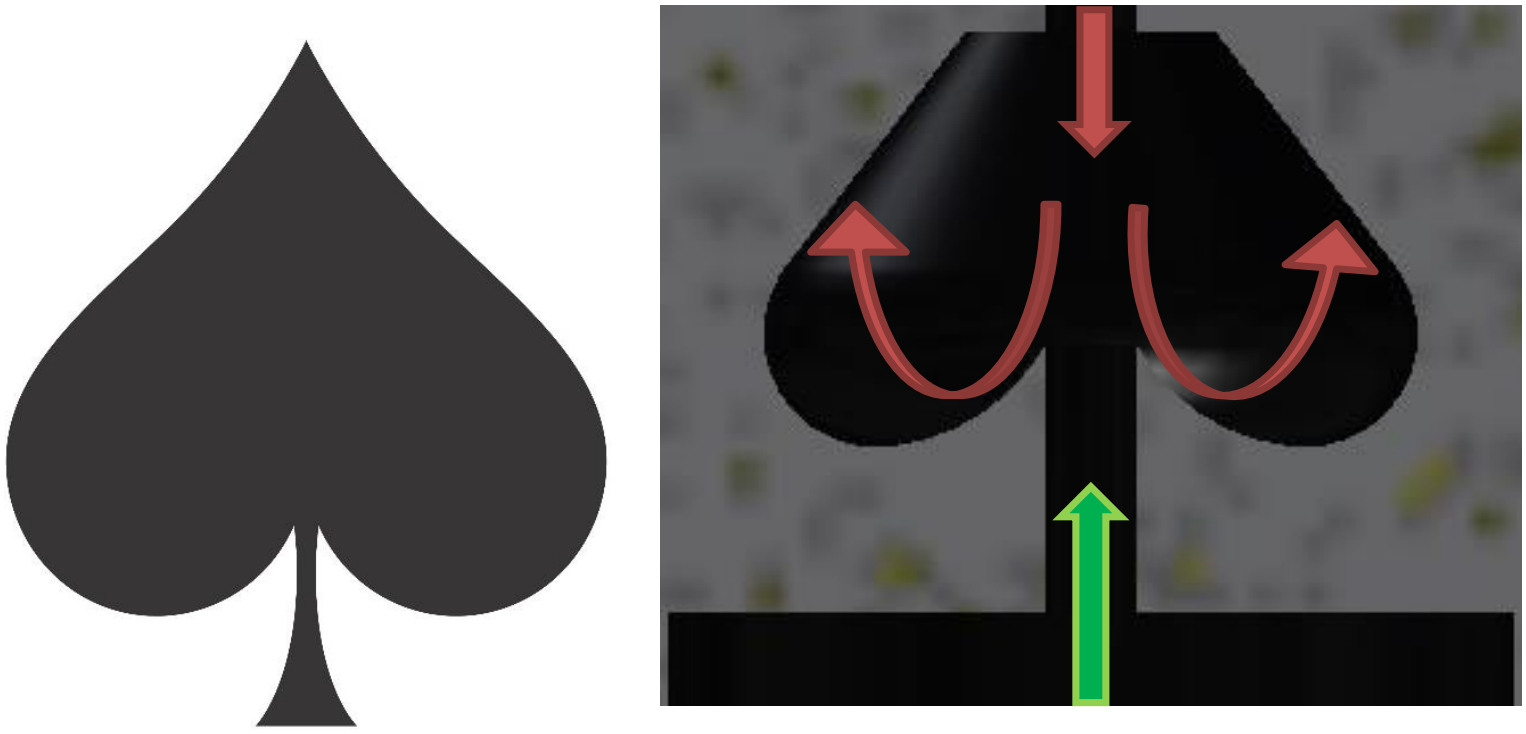

Figure 37. CAD view of fuel section modification made to each fuel injector

In reality, this geometry is a type of fluidic diode. They could in fact be used to stack one on top the other, and make a cascade arrangement. This is essentially what 
Tesla's valve is composed of [71]. They act as check valves for the flow-inhibiting it in the reverse direction, while having little or no effect on the forward flow. Theoretically, these type of valves could be employed on the air side as well. However, in this study, they are only tested on the fuel injection side. 


\subsection{Adjustable Reflector}

As a precursor to the reflector design with a split passage configuration, this design was conceived to allow a small plate to be inserted into the slots, which would allow the cavity length to be adjusted. This design also incorporates a small expansiondeflection (ED) before the air enters into a CD nozzle similar to baseline design. This is intended to deflect and help reject any flow that is traveling upstream through the inlet. The fuel injectors are unchanged in this design. The support struts for the ED nozzle were only a couple thousandths of an inch thick. They had sharp leading edges, and a thin profile in order to be less likely to induce shock reflections and minimize their influence on the inlet's transient flow behavior. They are staggered throughout the inlet test section, offset between the fuel injector locations. This design is shown in Figure 38.

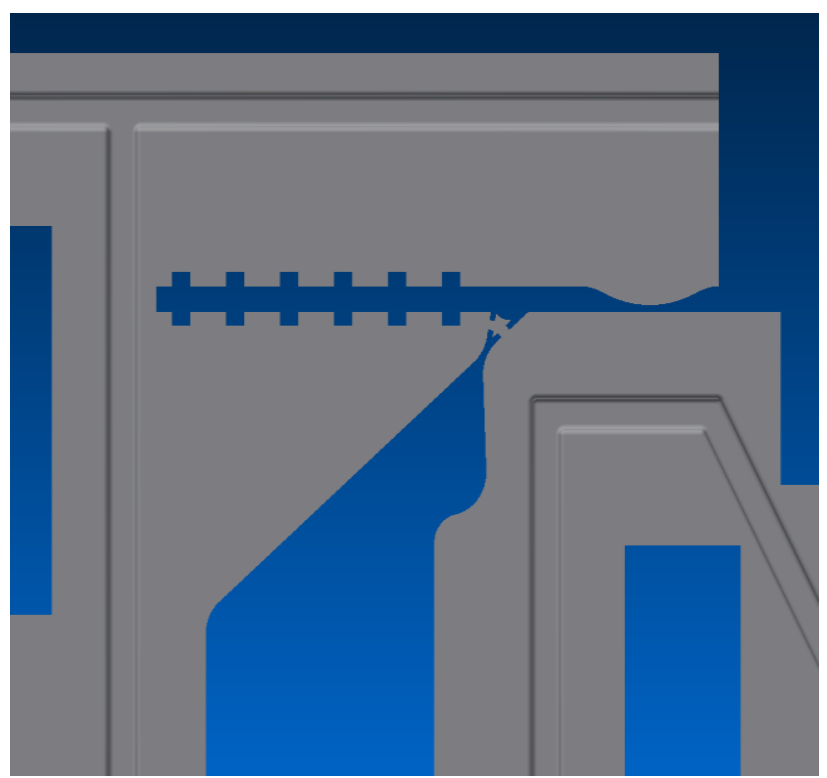

Figure 38. Adjustable reflector conceptual design 


\subsection{Shock Reflector Design}

Another design considered takes a multi-faceted approach to improve the inlet dynamics. It contains several unique features that are intended to target different issues with the inlet. Its main feature is a small rectangular volume that is directly perpendicular to the axial flow direction. This area is designed with a length so that a shockwave that begins traveling upstream through the inlet will have just enough time to reflect against the wall of the reflector, and travel back to the combustion annulus just in time for the passing of the next detonation wave. The evacuation of the shockwave from the inlet will allow for the fresh mixture to trail behind the shockwave and be delivered to the annulus. This is of course assuming that the frequency of detonation is a known, consistent value during operation. Otherwise, the timing of the reflections will be entirely out of phase, and the reflected waves will not serve its maximum potential purpose. The other distinct feature of this inlet design is the branching of the air inlet, which creates two discrete passages for the air. The fuel injectors are located between the two air streams. The full design is shown in Figure 39. The reason the air is split into two pathways is so that when backflow occurs, the air injection is the first stream to recover before the fuel injection. This should help avoid exceedingly fuel lean/rich pockets in the fresh combustible mixture. 


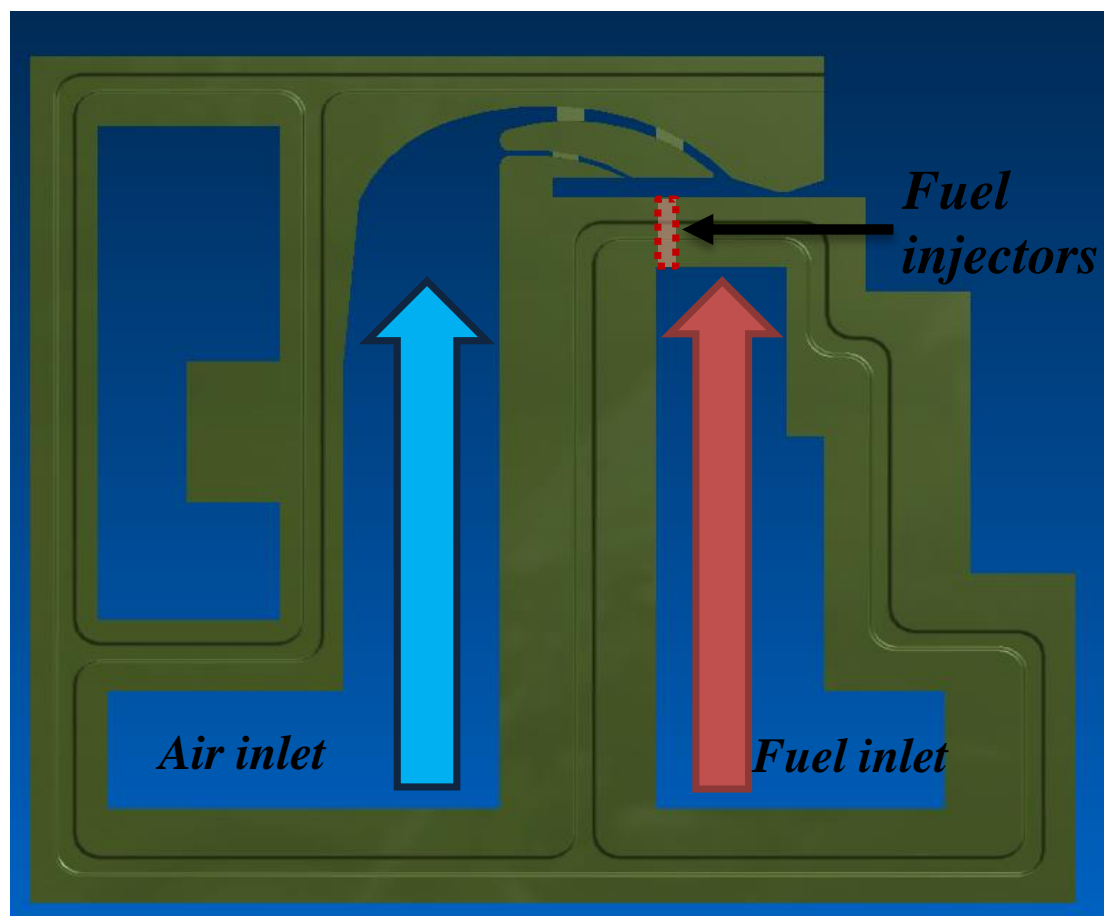

Figure 39. Shock reflector design mockup

The sizing of the cavity used for the reflector is based on the operation mode of the RDE. Ideally, this would need to be determined prior to manufacturing of a reflector inlet to test in the FSRDE. However, it is possible that the length of the cavity could be made such that it is adjustable, which would give good flexibility for this design over a wide range of operating modes. The main properties of interest that will drive the cavity length is the number of detonation waves, and the wave speed. A routine to calculate that length based on these inputs is included in Appendix B. A more accurate measurement of temperature in the cavity would be necessary, so that the speed of sound could be precisely known throughout operation. The support struts for the divider portion in the air streams were designed very similar to those used in the adjustable reflector design. Sharp leading edges, and a thin profile make them less likely to induce shock reflections and minimize their influence on the inlet's transient flow behavior. The sizing of the air streams was based on the CFD work that is covered in Chapter 3.4 Simulation Validations. Since the CFD results revealed that placing the primary air stream closer to the detonation annulus would yield quicker recovery of the total air stream, the design was created that way. 


\section{Chapter 5: Results and Discussion}

Three main testing parameters are presented in this section that are used to evaluate and compare each inlet design. Cases were tested in which the air gap of the baseline inlet was incrementally increased. Testing was done to evaluate how the feedback effect changes with increasing air gap sizes. Three different air gap inlets were tested-0.022", 0.044", and 0.066". These three inlets are not new designs, but rather iterations of the same design. Each of the three gap sizes resulted in different pressure ratio (PR) values, refueling time scales, and backflow length scales. As the air gap changes, the exit conditions of the $\mathrm{CD}$ nozzle change. This means that it will go from being choked at small gap cases, to not choked at large gap cases. Several key observations are made as the air gap increases.

From the data obtained, it is clear that there are two different classifications of flow reversal. Each of them have a different effect, and each must be controlled differently. One type of flow reversal is driven by the detonation front. It is characterized by the shockwaves that travel through the inlet. They move at a high speed, and are dissipated within less than half of a millisecond typically. The second category of flow reversal generally follows the detonation front, and is characterized by bulk fluid motion, which is mostly the result of the products of combustion. This region of combustion products is present in the inlet for a longer time period than the pressure peak that is induced by the shockwave. The time scales for these two types of backflow are significantly different. The shockwave is present in the inlet plenum for approximately a few tenths of a millisecond (depending on the inlet geometry), while the combustion byproducts appear to linger in the inlet until the forward flow is able to force it out. The characteristic time scale of the shockwave, $t_{s h}$, is much shorter compared with the characteristic time scale of the bulk combustion product flow, $t_{b}$. The residence time of the shockwaves in the inlet plenum is a critical measurement. The longer that shockwaves stand in the plenums, the greater the backpressure on the plenum that causes flow 


\section{Results and Discussion}

blockage. The inlet design procedure had to be approached from both perspectives, dealing with product isolation (from bulk flow) versus pressure isolation (due to shockwave propagation).

From Schlieren images taken, wave speed of the shocks in the inlet is calculated for the experimental runs. Over a given set of frames, the wave speed can be averaged. Once the shockwave decouples from the detonation wave, they will notionally be travelling at the speed of sound for air in the inlet plenum. However, since the shockwaves must first detach themselves from the detonation wave when traveling upstream in the inlet, there is momentum to the backflow that gives the shock front a higher velocity until it degrades to the sonic speed [69]. The instantaneous shockwave speed can be found from two successive frames. Since the time difference between frames is easily known, and the distance can be evaluated, the shock propagation speed is then calculated. The gridded structure in Figure 40 provides a reference length scale for velocity calculations. The propagation speed can be plotted as a function of the number of frames to find a function that fits the declination of wave speed, from detonation wave speed to sonic speed. This relationship is found to be fit accurately from a quadratic function. The purpose for finding the shock propagation speed is mainly applicable to the Busemann inlet design, for which the Mach number must be known for tuning the geometry of the Busemann inlet. The shock propagation speed determined from the images in Figure 40 is approximately $391 \mathrm{~m} / \mathrm{s}$. This is slightly greater than the speed of sound $(M=1.14)$, which is attributed to the momentum that the shockwave possesses after decoupling from the detonation front. The shockwave detaches from the detonation front, since the shockwaves travel upstream through the inlet, while the main detonation continues travelling circumferentially in the detonation annulus.

The strength of the shockwaves in the air plenum appear to be much stronger than those in the fuel plenum (comparatively). However, there are a greater number of coherent shockwaves traveling through the fuel plenum. This indicates that the fuel plenum may be more susceptible to the shock presence in its plenum. Since the speed of 
sound of hydrogen (and helium) is much faster than standard air, the shockwaves will propagate through the fuel plenum faster than the air plenum.
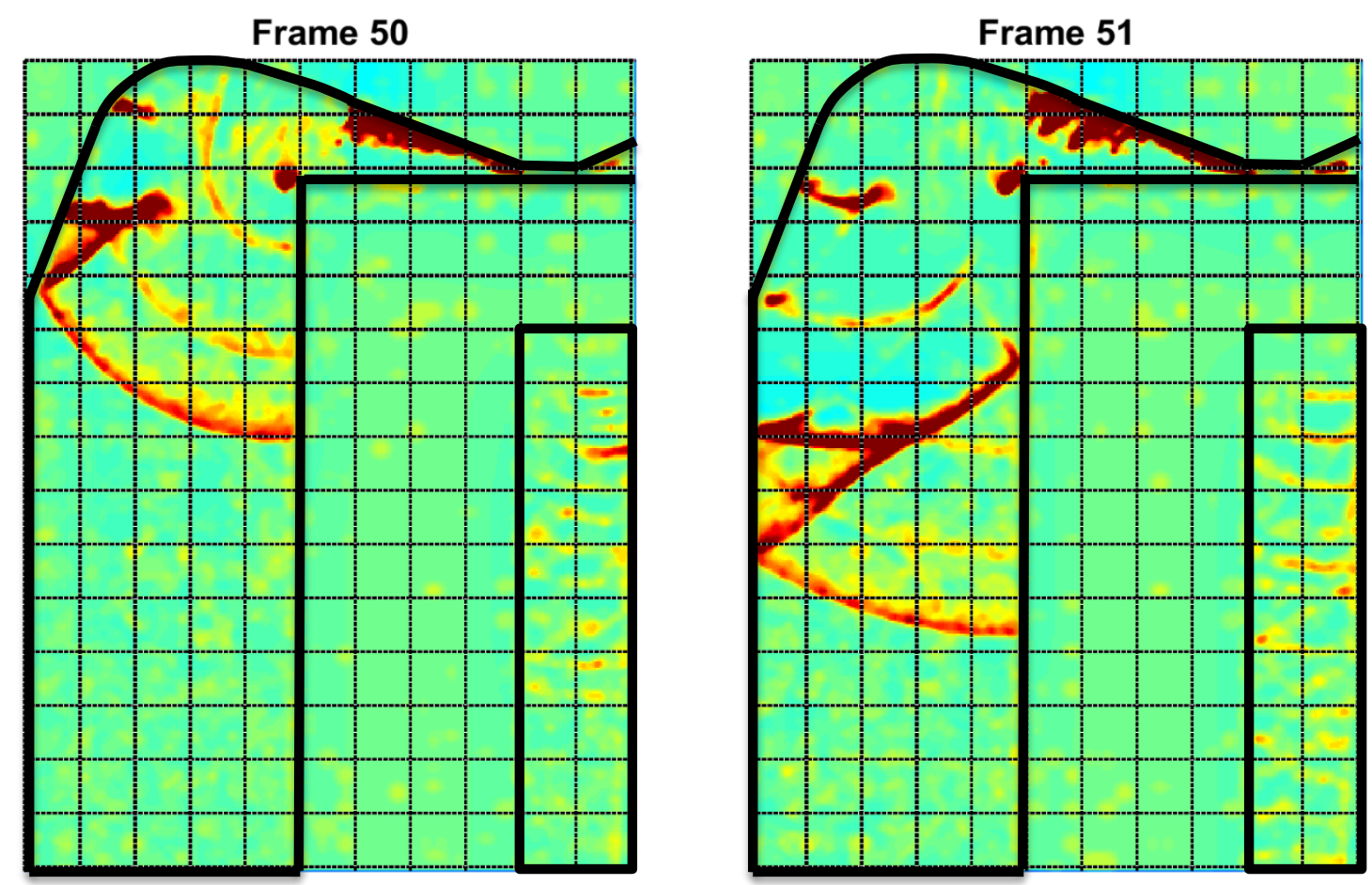

Figure 40. Two successive Schlieren frames of shock structure in inlet plenums, 0.022" gap

The presence of these shockwaves in the inlet plenum is alarming. Until significant dissipation takes effect on the strength of the shocks, they will notionally continue travelling upstream of the system. In the case where the RDE is integrated into a turbine engine, the shockwaves could cause damage to compressor components. Not to mention fluid-structure interaction with the compressor blades, that could lead to many structural issues. Shockwaves are visible in Figure 40, where the air and fuel inlet plenums are outlined in black. 


\subsection{Backflow Length}

The maximum backflow length, $L_{\max }$, can be measured using a MATLAB routine that relates the image resolution to a known length in the image. The known length is determined by fist referencing a feature of the geometry, of which a specific dimension is known in order to acquire the number of pixels per inch (ppi) in both the horizontal and vertical directions. Because there is not a distinct endpoint to the bulk fluid that is identified as the backflow, the method is not highly precise. However, it does provide a good approximation to the backflow vulnerability of each inlet. By tracing a line along the length of the products of combustion, the routine calculates a length of backflow (penetration into the inlet). This is demonstrated in Figure 41. The length that is expressed is measured from the datum of the centerbody wall (the inner surface of the annulus). The two clear champion designs in terms of backflow length are determined to be the wedge inlet design and the spade diode. The spade diode backflow was actually immeasurable, since there was no noticeable backflow events recorded. The wedge inlet reported almost a third of the backflow length that the baseline design reported. Figure 42 shows the backflow recorded for the wedge design. It is miniscule in comparison with the backflow exhibited in the baseline design.

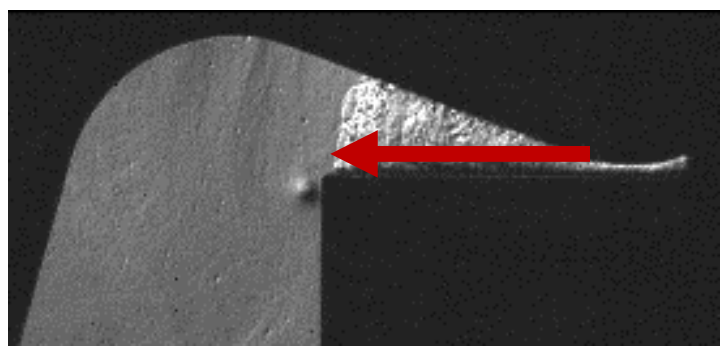

Figure 41. 0.044" Gap, 700 slpm, highest backflow induced 


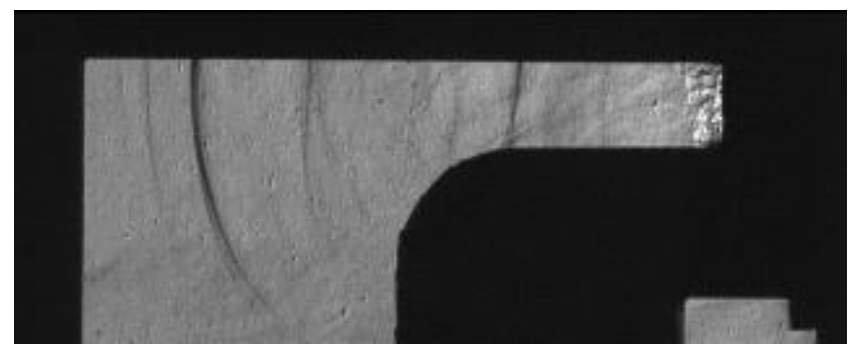

Figure 42. Wedge design, highest backflow experienced

Surprisingly, the backflow length for the remainder of the inlet concepts fell between the small and intermediate gap. The backflow length is a measure of the product backflow, as opposed to the pressure backflow. The flowfield is quite complex and convoluted for the adjustable reflector and split path design. It is difficult to decipher when the shockwave (which dissipates into a pressure front) can be distinguished between the bulk product flow. The transition from a shockwave to a pressure wave is discussed and described more in-depth for the adjustable reflector design. It is also difficult to track the backflow in the split flow path design, which can be seen in Figure 43. Since the flow travels through both curved, serpentine passages, it is not as accurate as calculating a linear dimension. Since strong gradients are imaged at the secondary air path (the smaller one) due to the expanding air, it again becomes challenging to interpret the backflow from the injecting air.

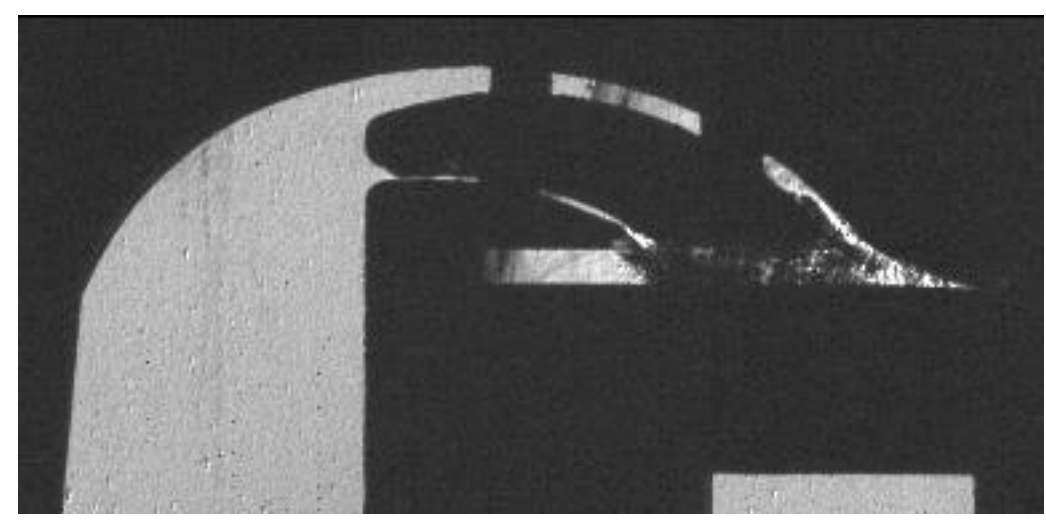

Figure 43. Maximum backflow for split path design 
A surprising observation from the Schlieren images is that the shockwaves do not have a substantial impact on the combustion products. However, the shockwaves do induce instability to the injectant flow. Figure 44 shows the decrement of the air jet velocity due to the shockwave. Starting at frame 3, as the shockwave passes through the injecting air, the air jet loses its velocity. The secondary air stream appears stagnant for a couple of frames, until the shockwaves finish traveling through the air path.

Unfortunately, the shockwave does not succeed in pushing the combustion products out of the inlet cavity. At frame 4, the shockwave is almost to the end wall. The remaining frames in the progression show the result of the reflected shockwave off of the cavity wall. There is no visible evidence of the shockwave driving out the combustion backflow. The shockwave travels directly through the combustion products, but has little to no energetic effect on the bulk of fluid.

The split path reflector design did not report well on its recovery time. This is slightly misleading, because the combustion products seem to remain in the reflecting cavity well after the detonation event. This means that it has extra combustion products to move back out of the injector. If this design were to be run at higher pressures and flow rates, the air streams would have sufficient mass flows to better force out the undesirable products.
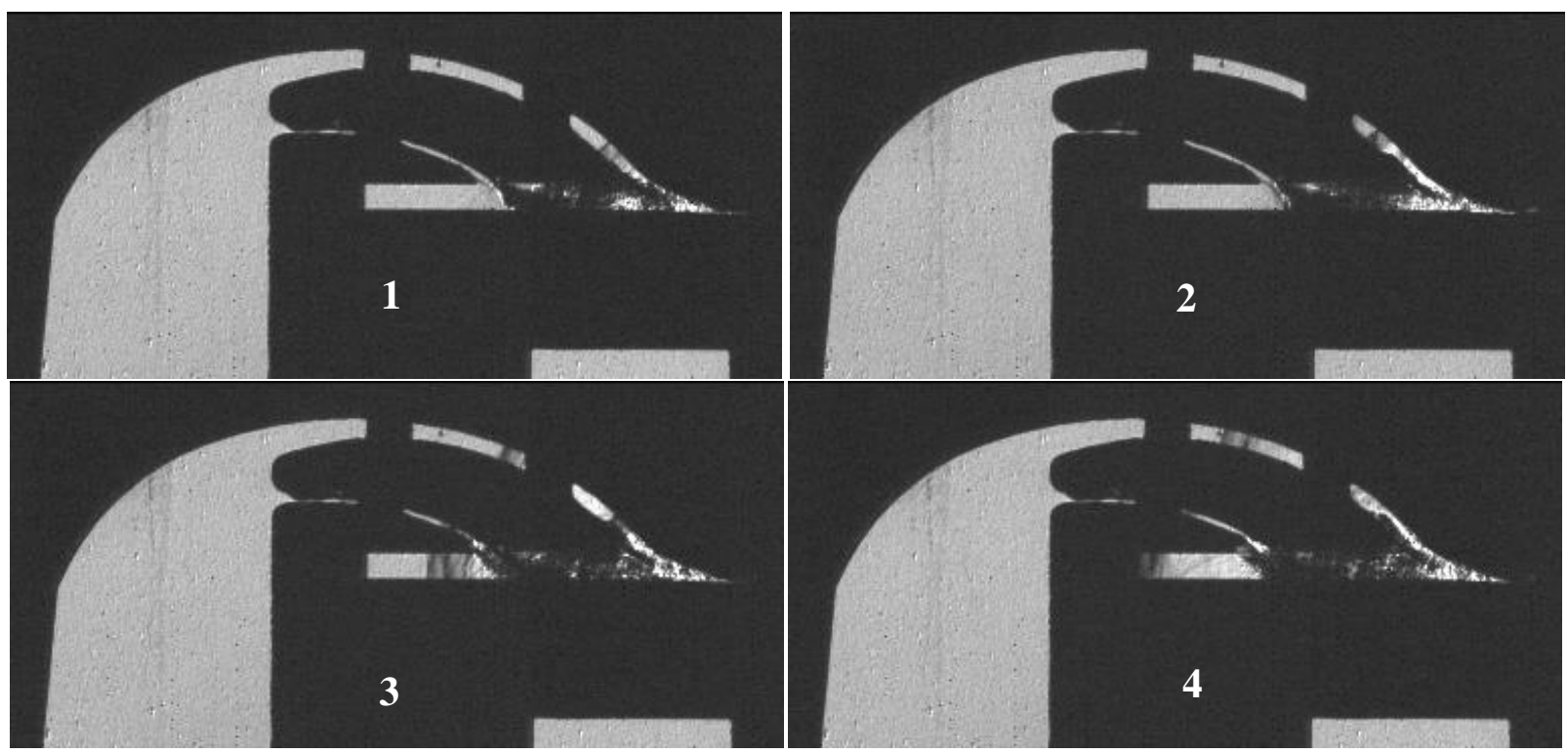


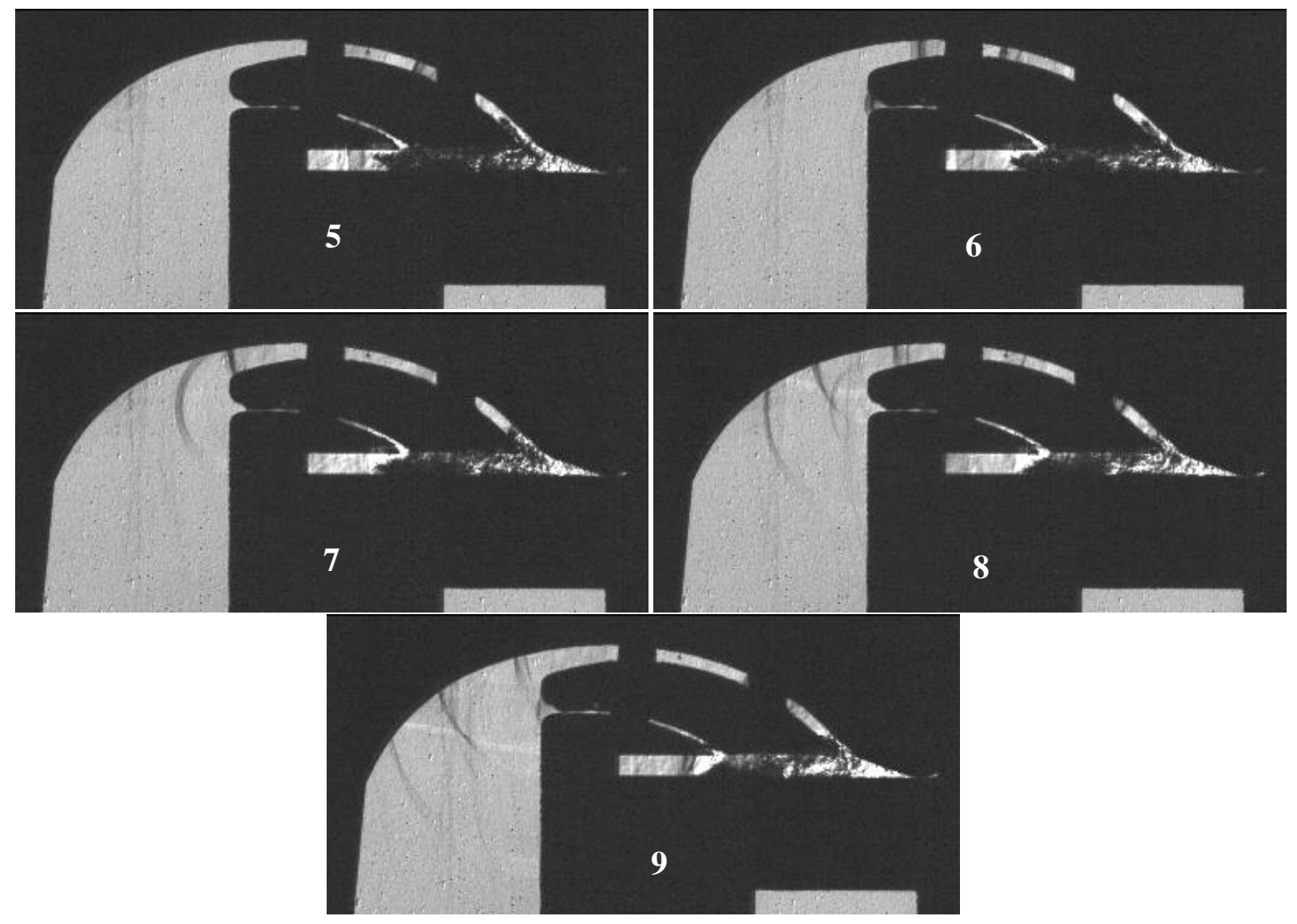

Figure 44. Frame progression of split path design

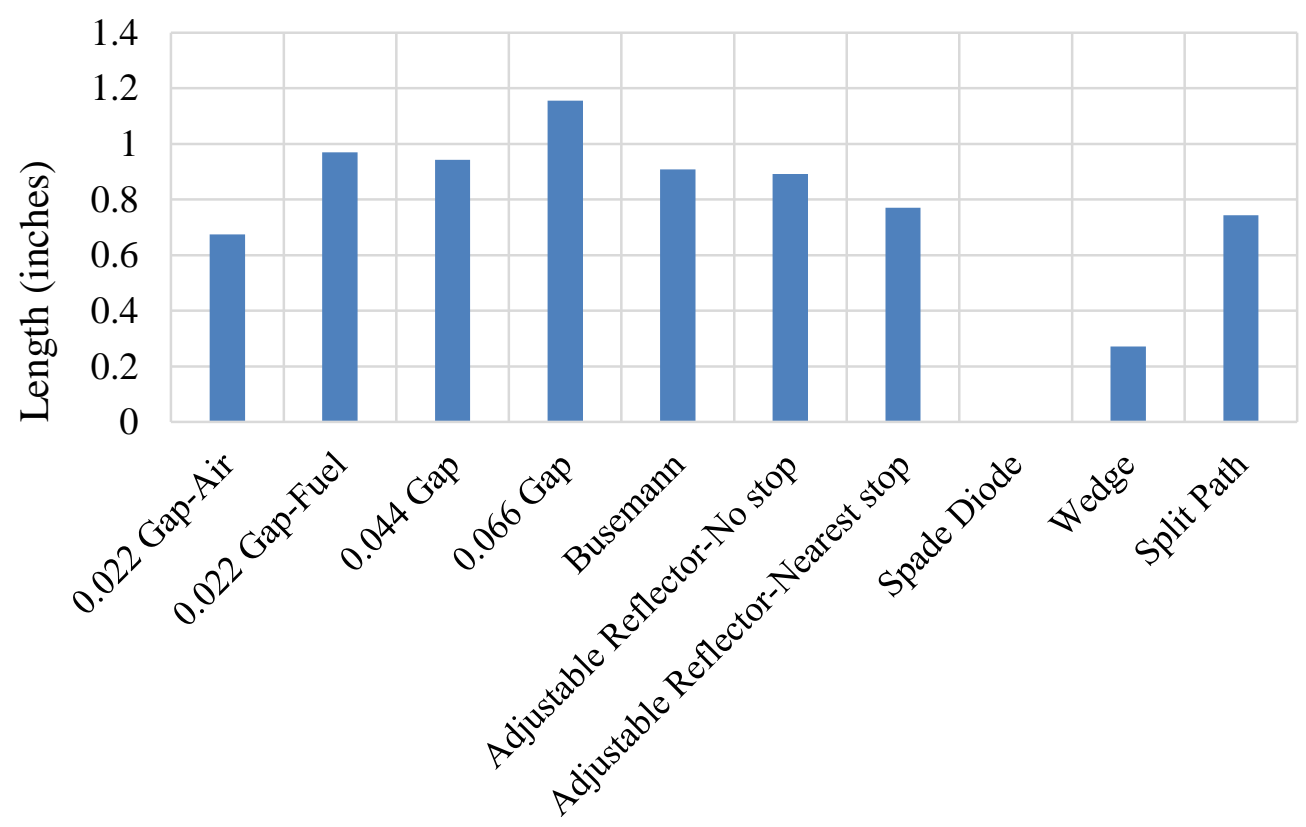

Figure 45. Maximum backflow lengths 


\subsection{Interruption Time}

From the pressure profiles in the air and fuel plenums, the interruption time can be calculated. This is the amount of time that the nominal pressure value in each of the plenums is overcome by the resultant pressures from detonation. Until the pressure in the plenum returns to its static level, the inlet flow is deemed interrupted. In other words, the interruption time is the duration of time that shockwaves remain detectable in the inlet manifold. This is perhaps one of the most important findings. The longer the interruption time is, the longer the inlet flow is affected and/or delayed. It causes inhomogeneities in local equivalence ratio, which leads to stability issues of detonation. The interruption time is considered the period in which the pressure in the plenum is above the set point for plenum pressure. Refueling does not occur during the interruption time, since this is the time in which backflow is occurring and the forward flow is prevented. The interruption time is a byproduct of the pressure backflow, caused by shockwave presence.

A frequency behavior can be extracted from the pressure profiles of each inlet. This provides knowledge about the natural frequency of each of the inlets, and where various frequencies get excited at. After the detonation wave hits the inlet section, the manner in which the shockwaves propagate and reflect within the plenums give a ring down effect to the pressure traces. A frequency analysis was performed for both the air and fuel plenum pressure signals. This provides information about how it corresponds to the detonation waves, but also what type of natural frequencies each geometry possesses. The spectral analysis in Figure 46 is from the smallest (0.022") gap inlet. The low frequency excitation, which has been observed by other researchers, can be confirmed. The low frequency signal, which is approximately $100 \mathrm{~Hz}$, is highlighted by the green region in Figure 46, and it is most likely a Helmholtz resonance of the design. Since it is present in all of the designs but the wedge inlet, it could potentially be a characteristic of the CD nozzle, which is incorporated into the majority of the designs. The fundamental operational frequency, $1250 \mathrm{~Hz}$, can be seen as an excited level. In addition to that, the harmonics of the primary frequency are also excited $(2500 \mathrm{~Hz}, 3750 \mathrm{~Hz}$, etc.). 

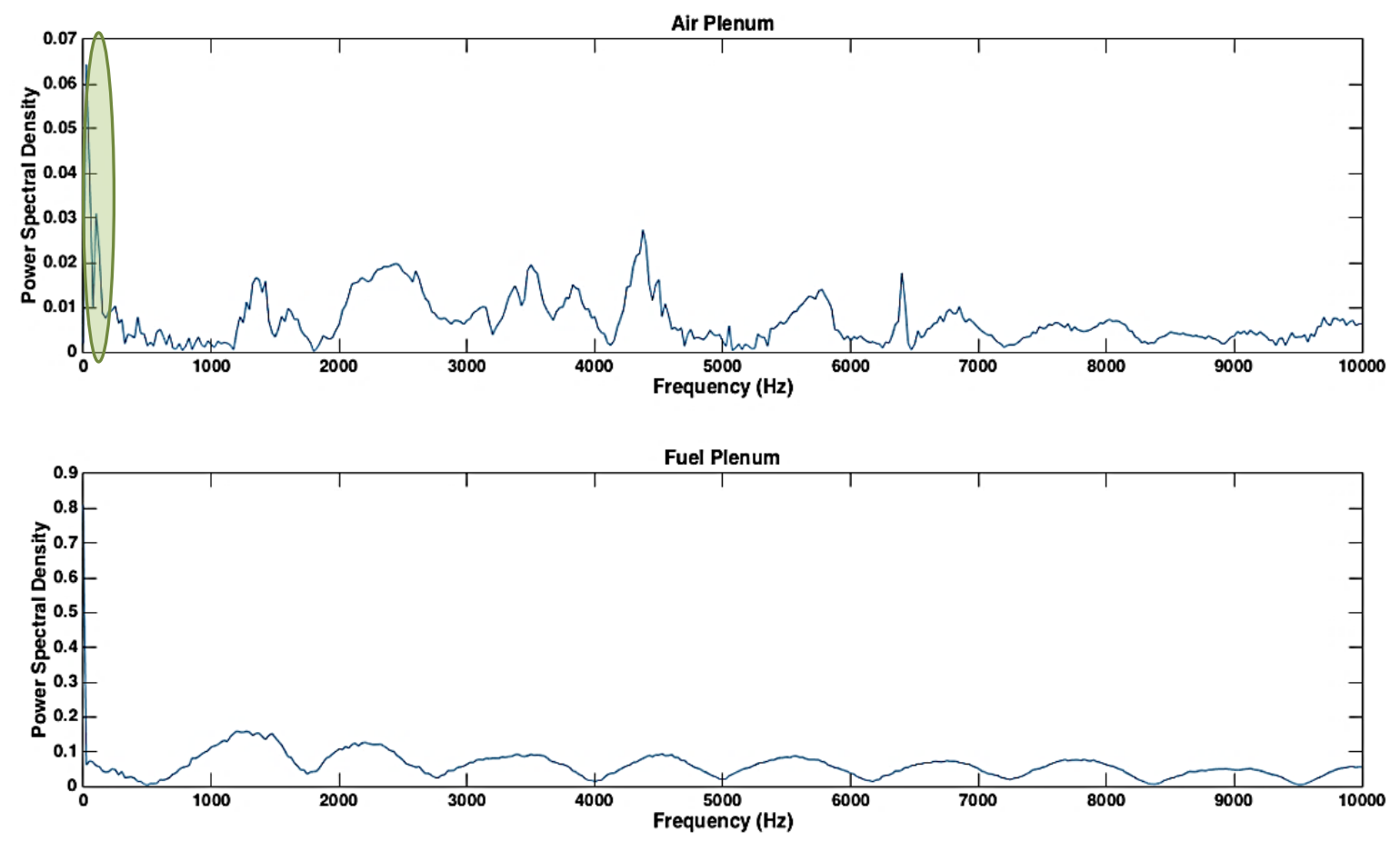

Figure 46. FFT spectral analysis of 0.022 " Gap inlet 


\subsection{Recovery Time}

The recovery time is determined from the Schlieren images of each inlet. While this is similar to the interruption time, it is not the same. The interruption and recovery times for a given inlet can potentially be the same, but they do not necessarily have to be. The recovery time is the amount of time that the injectant flow takes to force any combustion products out of the inlet area and back into the combustion channel. It is possible that the flow can be fully recovered, but still be interrupted by pressure waves. This is because reflections from shockwaves within the plenums can still cause the pressure to exceed the fixed static feed pressure, but the flow has already gained its forward momentum enough to recover. The recovery time is measured as the time from when the combustion products first appear in the inlet, until the time when the inlet plenum returns to its steady state refueling condition, at which no combustion products still linger in the inlet.

Early iterations of the inlet designs contained a cutout on the bottom of the fuel and air plenum. These cutouts were originally intended for visualizing from the bottom up through the plenums. Later versions of the inlets did not have these cutouts. A unique occurrence was observed between the inlets with the cutout versus those with it. On test inlets that did not have these features, there was a very noticeable strong shockwave reflection after the initial shock train travels entirely through the plenum. Different than the dark colored bands of the initial compression shock front, this reflection appeared light in color. Since the Schlieren image corresponds to differences in density, it is believed that the reflection was actually observed as rarefaction waves [72]. In some images, the dark and light bands could be seen coexisting, showing the leading compression front followed by the expansion post-wave. This was an encouraging phenomena, which is worth considering in all future inlet designs for the FSRDE. However, it does tend to suggest that the oxidizer injection would be better suited axially, as opposed to tangential. When trying to reflect shockwaves back through the exhaust, tangential injection schemes tend to direct the shockwaves directly against the outerbody, 
which then reflects them back through the inlet. These reflections continue recurring until the shockwave has been damped out. If the injection scheme were to be oriented axially, the shockwaves would travel through the entire length of the detonation annulus where it would be carried to the combustor exhaust. Radial injection schemes only tend to bounce the shockwaves back and forth against the combustor outerbody.

The data processing routine has many built-in functions that serve to analytically evaluate and report several key parameters that are extracted from the pressure data. Each data set has a search window that is set, throughout which the data is interrogated. The data that is analyzed is taken from a window of time, which encompasses the start of the detonation wave, plus 0.375 milliseconds from that point. This search window, in which the data analysis effort is focused, is highlighted by the green rectangular regions in Figure 47. This is so that anomalies (that are not a result of the detonation events) are not considered in the data processing. The ring down pressure signals read a lot of noise from the many shock reflections occurring within the plenums. The main point of interest to the inlet is what happens as a direct result of the detonation wave. To calculate the pressure ratios, the peaks of the pressure signals must be identified. The maximum pressure peak within that search window is chosen as the candidate for calculating pressure ratios. The data reduction routine finds the peaks in each pressure reading by looking for points above a threshold, which is set at the mean of the data, plus 2 standard deviations. There is some uncertainty with the detection of the true Von Neumann peak pressures that are measured. Since the majority of the data is acquired at $250 \mathrm{kHz}$, that is not a sufficient sampling rate to reliably resolve everything that is occurring within detonations. However, this sampling rate was limited by the throughput of the DAQ. Therefore, it is possible that the magnitude of peak pressure values may not directly reflect what the detonation is exhibiting. 

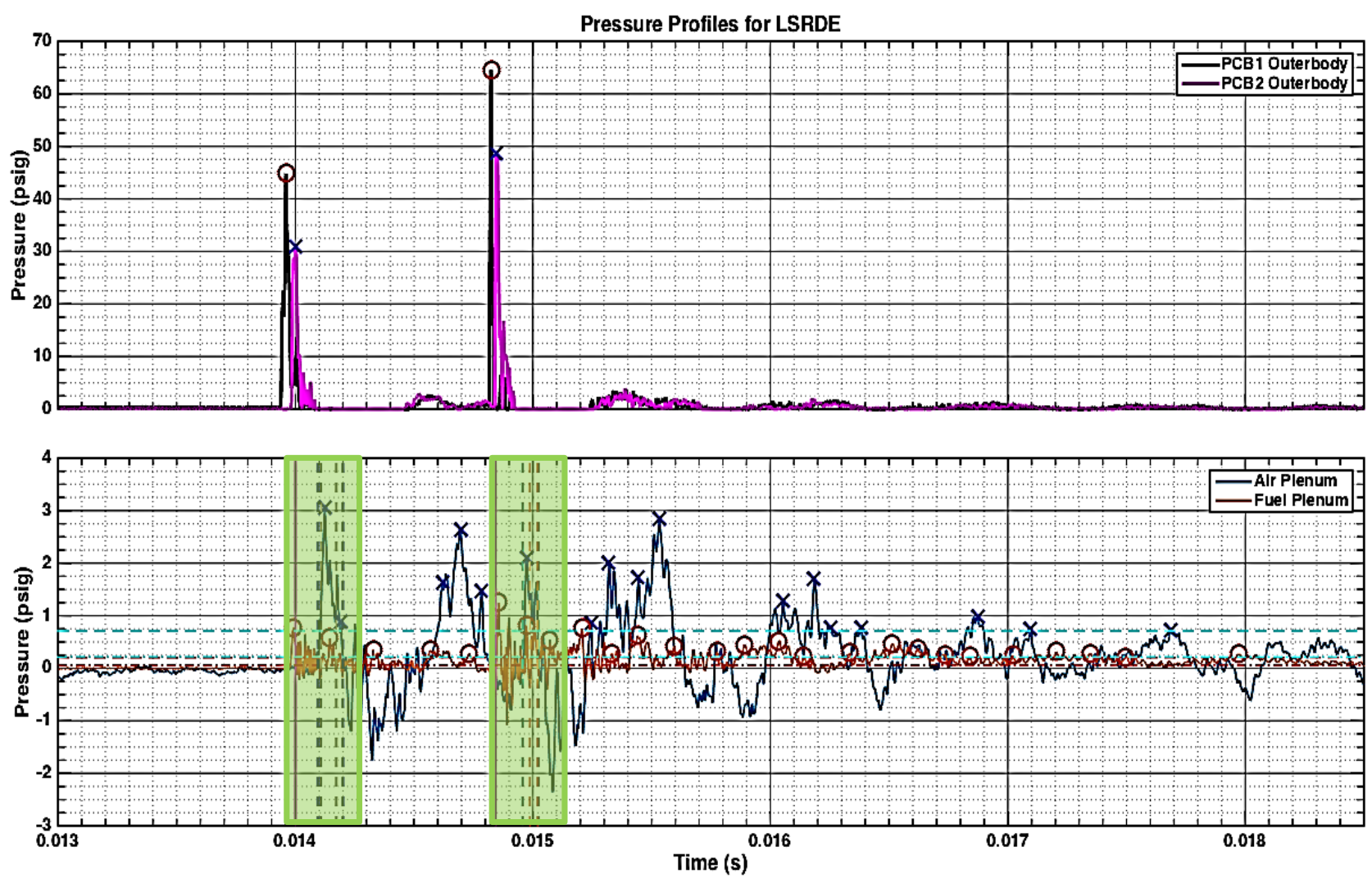

Figure 47. Data set from 0.044" Gap, $700 \mathrm{slpm}$, with interrogation windows indicated by green regions

The increase in air gap sizes resulted in many interesting observations and findings. As expected, the PR of the inlet increases with increasing gap size. Since the gap becomes larger, the inlet experiences a higher percentage of the peak detonation pressure feeding into the inlet plenum. Figure 48 qualitatively shows the increase in backflow length from the combustion products as a function of the air gap size. The interruption time for the 0.044 and 0.066 gap was very close, which could indicate that there may be a plateau at which the size of the air gap no longer changes the magnitude of the shockwave's influence. The pressure ratio increases almost linearly with the air gap. As air gap increases, the communication between the inlet and detonation channel increases to the same degree. 


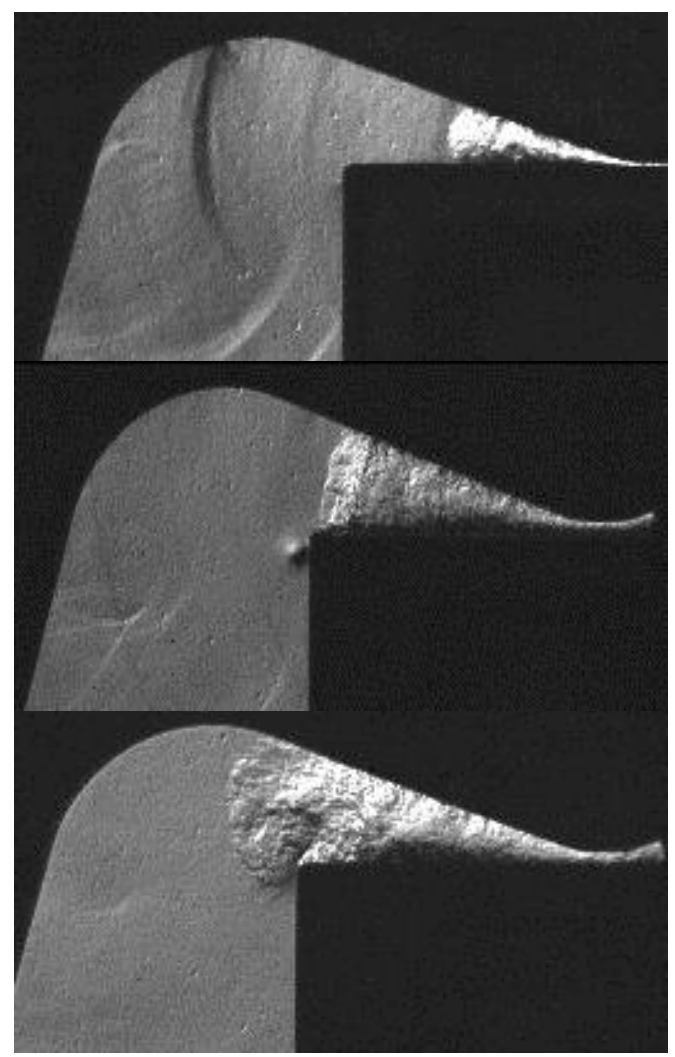

Figure 48. Maximum backflow shown for 0.022 " gap (top), 0.044" gap (middle), and 0.066" gap (bottom)

The adjustable reflector design proved to act more as a filter than it did a reflector. The slots that were built into the cavity for the adjustable wall caused significant damping to the shockwave. In fact, the shockwave was almost completely dissipated to a pressure wave by the time it reaches the back wall. This is shown in the frame progressions in Figure 49, where the reflector is shown without any wall stop in place. Unintentional in the original design, the slots for adding the adjustable wall act in accordance with a low pass filter [70]. The shockwave is indistinguishable by frames 5 and 6 . The pressure ratio values for the adjustable reflector were drastically reduced, which is reflected in the data summary section that follows. This is because the shockwave dissipation through the length of the filter reduces some of that pressure that has the tendency to be pushed through the inlet throat. The ED nozzle design also had a role in this PR reduction. Two 
wall positions were tested with this design. One with a wall at the closest position to the annulus, and one without any added wall. The design with the wall closest to the annulus performs better in all regards. This is likely because the shockwaves and combustion products push through the throat, and immediately hit the wall and turn back towards the annulus.

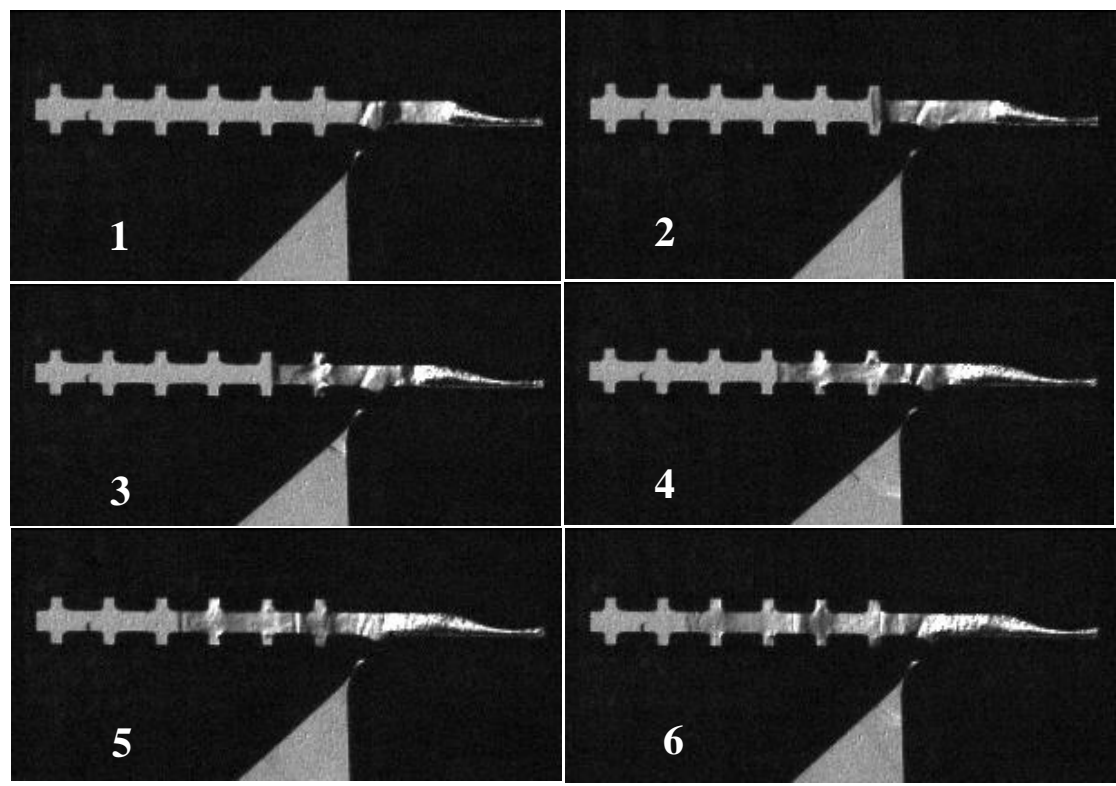

Figure 49. Evolution of shockwave through adjustable reflector, frame progressions at 0.01 seconds

The Busemann design performs well in several unique regards. While it does not appear to fully and successfully cancel the shockwaves as the design is theoretically supposed to do, it does seem to have a quenching effect on the backflow. Since the temperature in the inlet is not known, a marginal error in the Mach number could account for the design point neglecting to cancel the shockwaves. The Busemann design may be challenging to control the inlet flow during a RDE start-up transient. Upon light-off, the gas temperature will likely vary as wall heating occurs. This will cause Mach number to constantly change as temperature changes. Until the combustor reaches a thermal equilibrium, the Busemann design conditions may not be satisfied. The spatial resolution of the Schlieren images is not high enough to see the transitioning of the shockwaves through the throats. Therefore, it is difficult to say if any cancellation effect did reduce 
the strength of the shockwaves. There is a $10 \%$ reduction in pressure ratio from the baseline design. Since the pressures are mostly recording the dynamic pressure effect from the shockwaves, it stands to reason that the Busemann design was effective to a degree at reducing the shockwave strength. The maximum backflow condition for the Busemann design is shown in Figure 50.

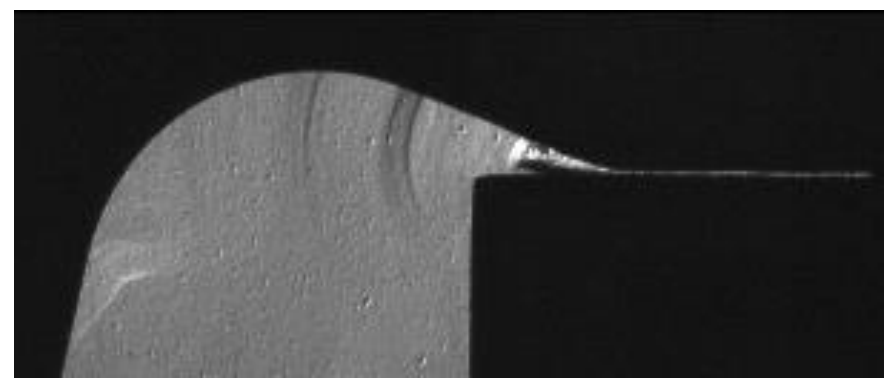

Figure 50. Busemann design at maximum backflow conditions

The wedge injector exhibited sensitive behavior to reflections in the opposite direction of wave propagation. As the detonation wave exited the channel, a shock reflection due to the sudden expansion was transmitted back upstream. It was determined that this high amplitude signal response was due to the reflection from exiting the channel based on its time difference after the pressure wave passed. This is made clear in Figure 51. The high amplitude responses that peak near 6 psig at 0.0148 seconds and 0.0156 seconds are well after the detonation waves have passed through the channel ( $0.8 \mathrm{~ms}$ after detonation waves have traveled out of the test section). The data for the wedge design misrepresents what would actually occur in an RDE. This reflected shock wave is traveling in the opposite direction that the detonation wave propagates in. This means that the wedges are facing directly into the oncoming shockwave, and a high pressure is fed back into the inlet. This is visible by the two pressure peaks around 6 psig in Figure 51. This phenomena is supported in the fact that these events occur well after the detonation is initiated. The detonation has long been evacuated from the channel when these highamplitude pressure signals are recorded. This is indicative of the reflection due to the 
shock expansion at the exit. In fact, this occurrence has some interference to the second detonation wave that is sent through the channel. From Figure 51, it is seen that the first high pressure spike, seen around $0.0148 \mathrm{~s}$, is passing through the plenum just before the second detonation wave passes. This is bound to have undesired interference effects. If this design were to be tested in a full 3D, cylindrical combustor, these effects would most likely not be observed. The fuel injectors exhibited moderately improved behavior over the baseline fuel injectors. Since they are shielded from the detonation wave in some degree by the wedges, this is consistent with the application of the ramps. The wedge fuel injectors recorded lower average PRs, as well as slightly shorter interruption times. 

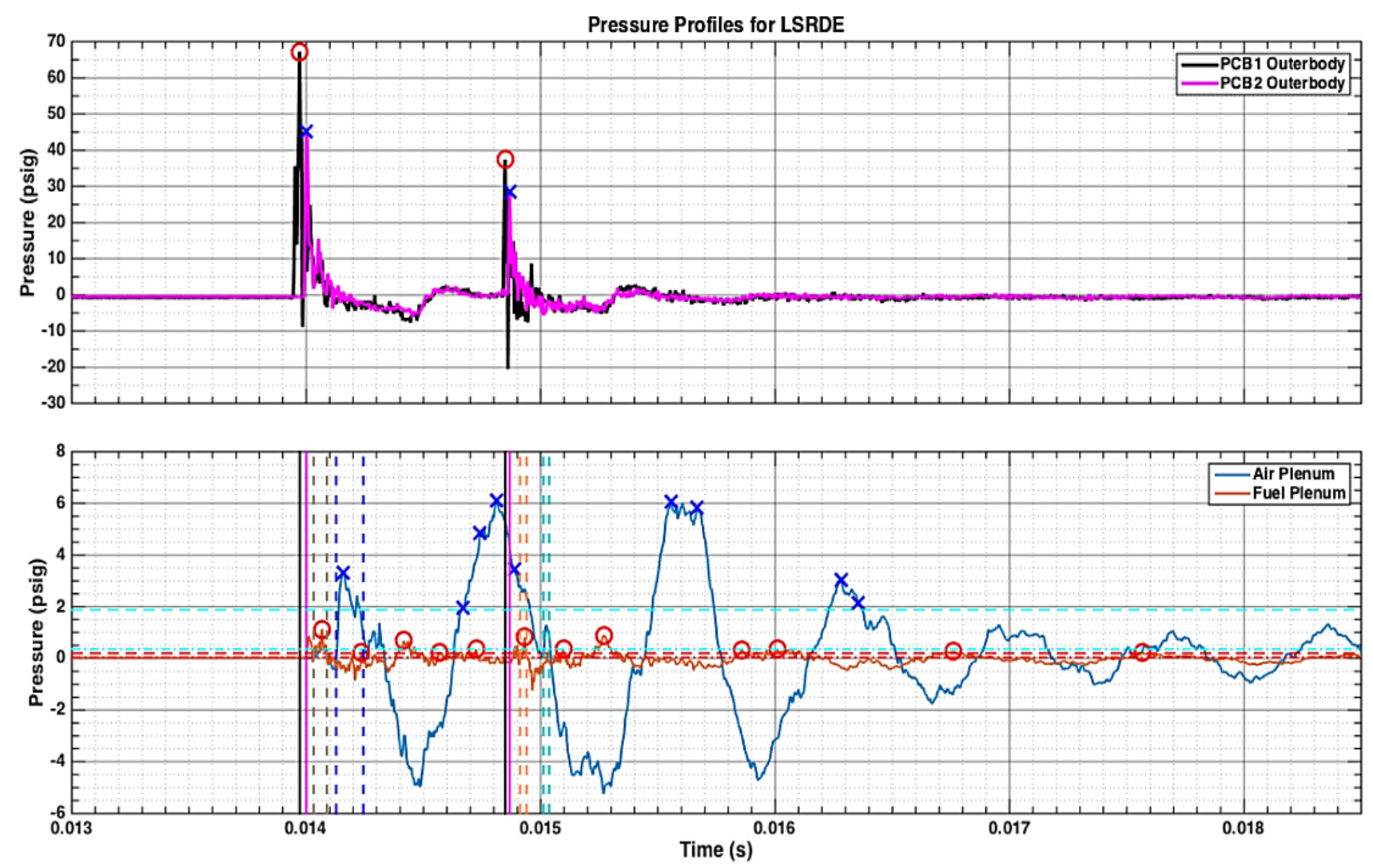

Figure 51. Wedge inlet pressure trace, 700 slpm case

The wedge inlet does a noteworthy job at reducing the low level resonant frequency commonly observed in the baseline inlet design. The drastic change in design changes the natural frequency of the injector, and does not excite the low frequency ranges seen in the baseline design $(\sim 100 \mathrm{~Hz})$. The FFT plots in Figure 52 illustrate this point. For the reasons discussed in the preceding paragraph, the spectral density of the $1250 \mathrm{~Hz}$ signal is unrightfully higher than what the expected data should reflect.

However, the harmonic frequency signals seem to be removed from the inlet operation. This is the main new inlet design that successfully changed the frequency response to a significant degree. The average channel Mach number increased for all tests of the wedge design. Since the wedges present an obstruction in the channel, the flow accelerates to move past the obstacles. 

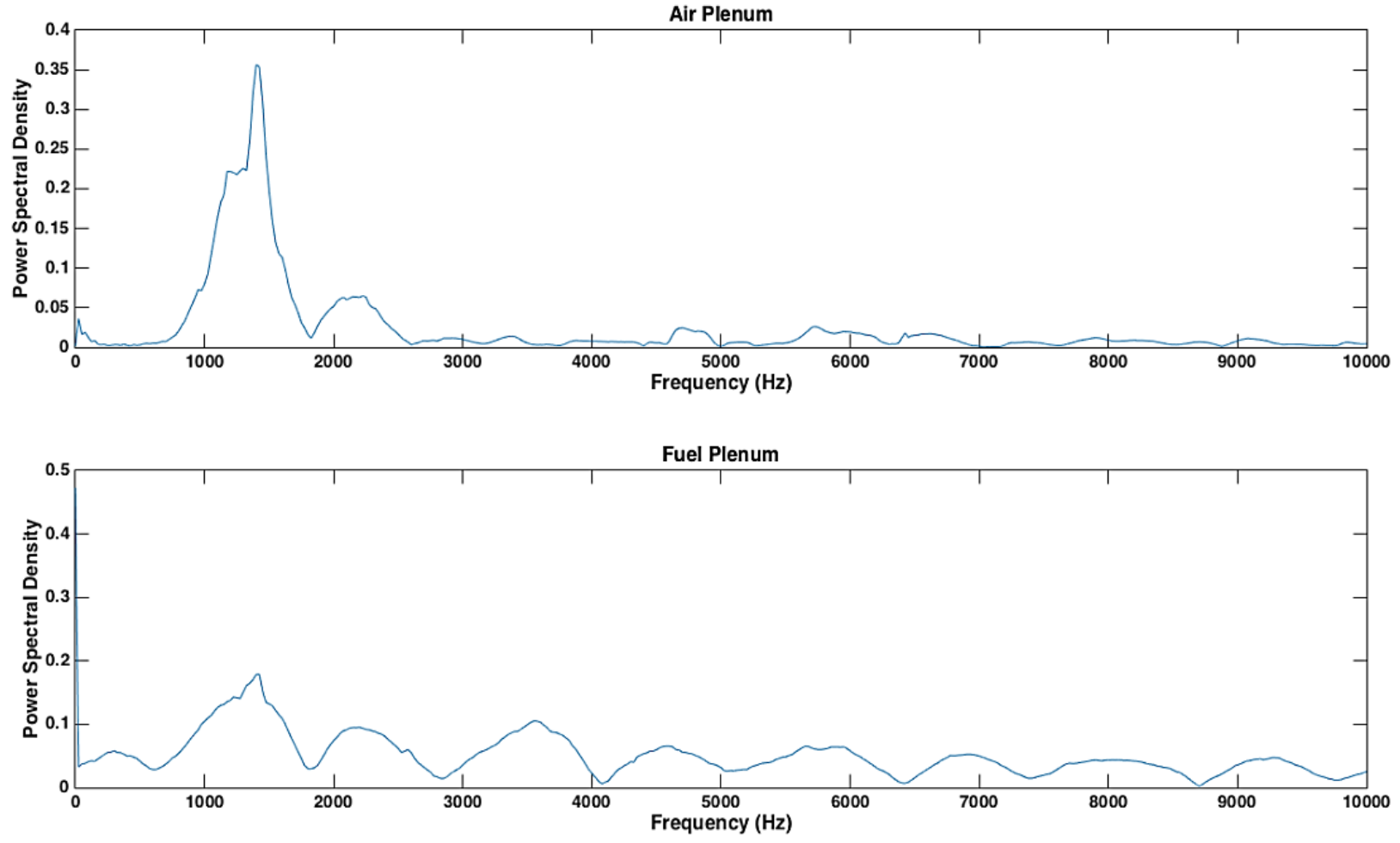

Figure 52. FFT analysis of Wedge design 


\subsection{Data Summary}

Each inlet design has a specific pressure drop that is induced across it. The amount of flow restriction is unique to each inlet design. The plot of volumetric flow rate versus inlet pressure is shown in Figure 53. The 0.022" gap has the largest pressure drop, in addition to the wedge and adjustable reflector design, which are very comparable. The increased gap size inlets $(0.044 \& 0.066)$ have lower pressure drops. The Busemann and split flow path designs have moderately pressure drop values. The wedge design, which shows a promising capability as a candidate design, does induce a high pressure drop that is comparable with the baseline design. However, its flow path through the plenum was not designed or optimized for pressure drop. It is speculated that the flow restrictions can be reduced notably with minor changes to the way that flow travels before getting injected into the annulus.

The spade diode shows many promising characteristics. It does not show any visual signs of feedback from the detonation. Therefore, it does not take any appreciable amount of time for the flow to recover. The interruption time is cut in half from that of the baseline fuel injection geometry. The pressure drop remains unchanged in the forward direction, unless the diameter of the main hole changes. This design can be extensively refined to be more useful and applicable to detonations. By tuning the volume of the expanded region and the angles of the turning faces, this design can be further optimized. While it was not tested in this work, it is suggested that this geometry be adapted to the air injectors (whether offset and staggered, or stacked on one another). 


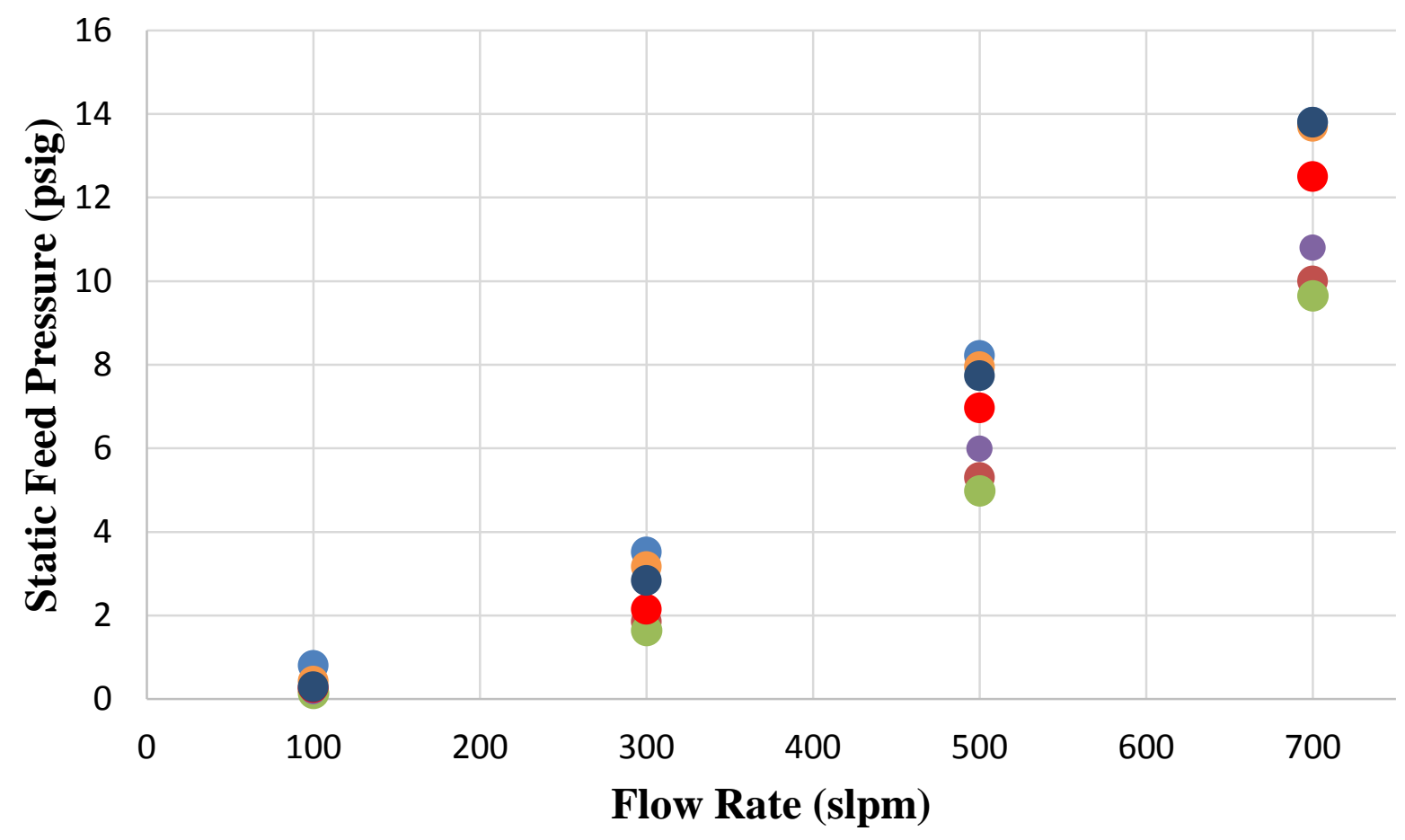

0.022 Gap

0.044 Gap

0.066 Gap

Busemann

Flow Split Path

Adjustable Reflector

Wedges

Figure 53. Variation in pressure losses of each inlet design

The distribution of pressure ratios for each inlet design shows some potential candidate designs. The pressure ratio for the second detonation wave is less than the first wave's for most of the inlets. This is explained because the shockwaves from the first detonation wave passing are still present in the inlet plenum, and damp out the second set of waves to some degree. This is supported by the fact that this does not occur for the wedge design. Since the initial shockwave passing is deflected off of the wedges, the only major shockwaves to enter the inlet are from the reflected ones after exhausting from the channel. Since there is a lag and time shift in this shockwave with regards to the ones from the second wave, there is no direct interference of the shockwaves. That is why there is no discrepancy between the first and second set of pressure ratios. While the 
wedge design pressure ratios are higher in value than expected (based on promising data with the backflow length and interruption times), some of it could be an artifact from the phenomena explained earlier. The benchmark data to improve upon is based on the baseline inlet, and is indicated by the dashed lines. The Busemann, split path, and adjustable reflector designs all show distinguishable improvement. The Busemann design shows improvement of around $10 \%$ in reducing the feedback pressure. The adjustable reflector design shows improvement of up to $50 \%$ of PR reduction.

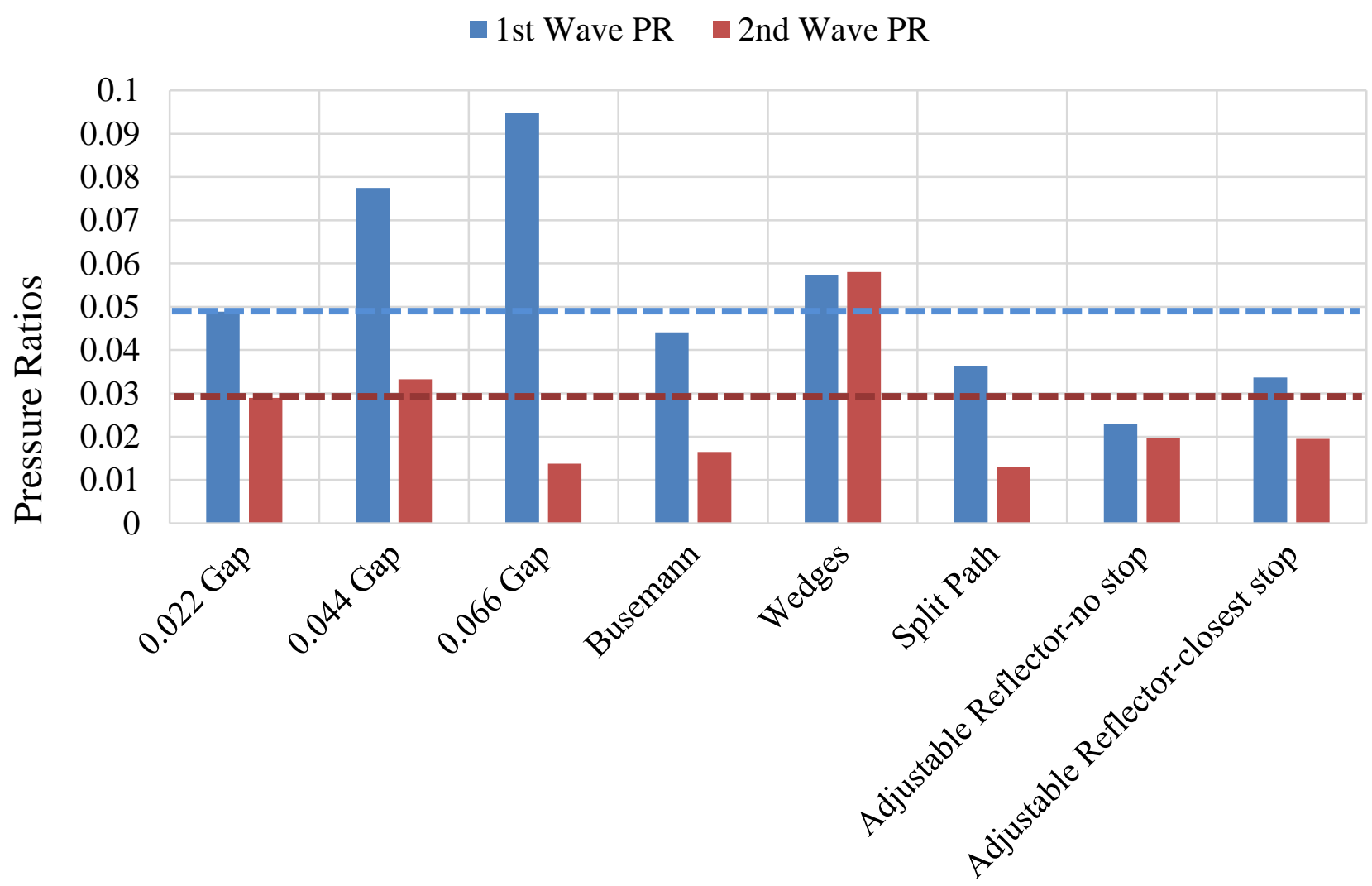

Figure 54. Pressure ratios for both detonation events

The spade diode design leads to a pressure ratio decrease of almost $55 \%$ on the first wave, and approximately $65 \%$ on the second wave. The spade diode averaged a reduction in pressure ratio of over $50 \%$. This could mean considerable improvements for 
a set of fuel injectors with this geometry. It could drastically reduce refueling and fuel interruption times, which would lead to more stable fuel injection and an overall stabilized RDE.

- PR 1 PR 2

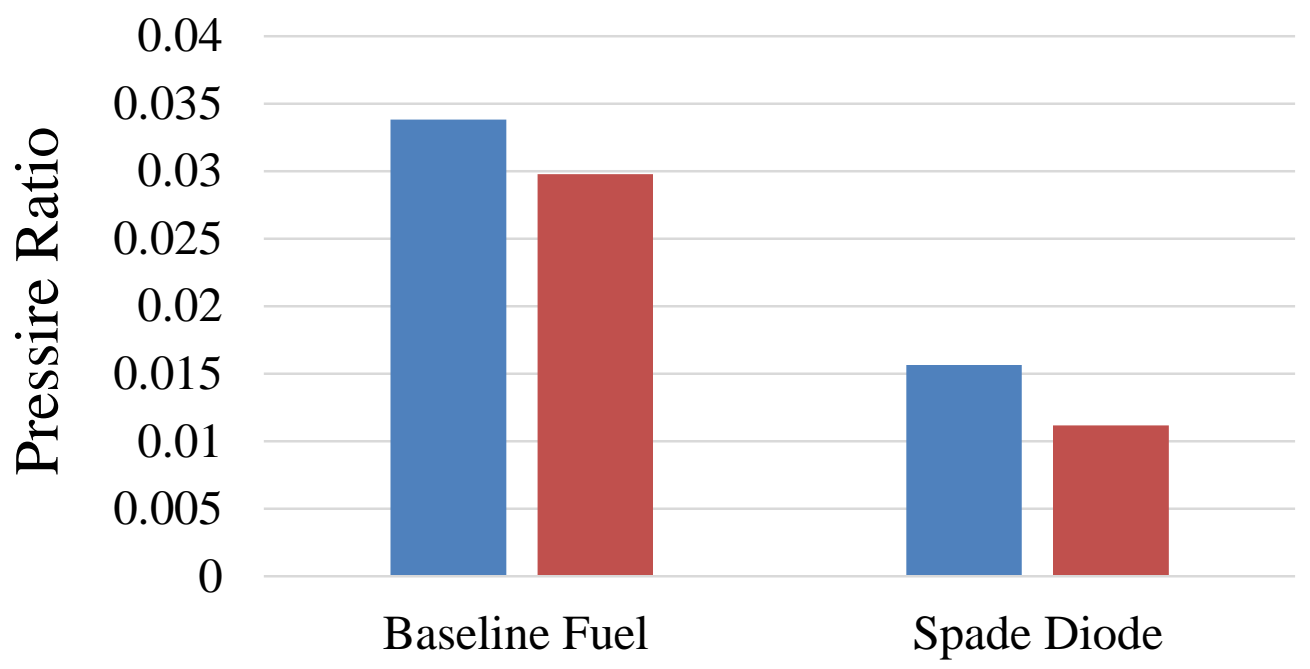

Figure 55. Pressure ratios for fuel injector designs

A summary of the data collected from all of the inlets tested can be presented in a comprehensive table, like the one shown in Table 2. It provides a good report of the backflow length, interruption time, and recovery time. It proves that it is difficult to drive improvements on all of the inlet metrics. For instance, the adjustable reflector designs both show a reduction in recovery time, but an increase in the backflow length. However, until these specific designs are tested or run in a detonation environment, it is challenging to state which is more important. The backflow length may be irrelevant to the inlet, so long as the recovery time is improved. 


\begin{tabular}{|c|c|c|c|}
\hline Inlet Design & $\begin{array}{c}\text { Recovery } \\
\text { Time }(\mathbf{m s})\end{array}$ & $\begin{array}{c}\text { Interruption } \\
\text { Time (ms) }\end{array}$ & $\begin{array}{c}\text { Backflow } \\
\text { Length (Lmax), } \\
\text { inches }\end{array}$ \\
\hline 0.022" Gap - Air Plenum & 0.300 & 0.0740 & 0.675 \\
\hline 0.022" Gap - Fuel Plenum & 0.320 & 0.0833 & 0.969 \\
\hline 0.044" Gap & 0.430 & 0.0860 & 0.942 \\
\hline 0.066" Gap & 0.600 & 0.0860 & 1.155 \\
\hline Busemann Inlet & 0.300 & 0.0479 & 0.908 \\
\hline Reflector with Adjustment (no stop) & 0.270 & 0.0467 & 0.892 \\
\hline Reflector with Adjustment (closest stop) & 0.240 & 0.0380 & 0.770 \\
\hline Spade Diode & 0 & 0.0420 & Undetectable \\
\hline Wedge Design & 0.210 & 0.0740 & 0.272 \\
\hline Shock Reflector-Split Passage & 0.486 & 0.0573 & 0.743 \\
\hline
\end{tabular}

Table 2. Data Summary for experimental inlets

The only geometry changes for the fuel injectors that were changed was the spade diode injector. However, the fuel injector dynamics did change with each changing air design. With each iterative air geometry change, the geometry of the fuel injectors remained constant. The only thing that changed was its position relative to the annulus. These changes were minimized, but done as needed to accommodate the design. Therefore, any changes in fuel injector response that was measured was simply due to the interaction with the new air geometry. It was determined that the change in air gap size had no effect on the fuel injector performance. For the 0.022, 0.044, and 0.066 gap inlets, the average pressure ratios for the fuel injectors were 0.0296, 0.0294, and 0.0246, respectively. It can be concluded then that the size of the air gap has a minimal effect on fuel injector behavior. However, for other air geometries, the fuel injectors exhibited significant changes in backflow resistance. For instance, the fuel injectors in the Busemann design recorded an average fuel injector pressure ratio of 0.0161 , a $46 \%$ increase from the baseline fuel injection scheme. The split path geometry recorded an astonishing 0.007 pressure ratio, a $76 \%$ increase from baseline. It is encouraging to see this improvement, since a major design intent for the split path design was to ensure 
better timing of the fuel and air injection. Granted, the injectors for this geometry are much farther back from the annulus, and protected by being inboard of the air throat. The results are encouraging nonetheless. The adjustable reflector design with no stop, and with the wall farthest outboard measured average pressure ratios of 0.0167 for both. The wedge design fuel injectors recorded a 0.0232 pressure ratio. This is a small improvement from baseline. Although this is close to the baseline design, the positioning of the injector must be considered. The fuel injectors in the wedge design are actually on the fuel plate, directly in the annulus. Their exposure to the detonation wave is much higher than the baseline fuel injectors. 


\subsection{Schlieren Imagery Processing}

The Schlieren images that are captured require rigorous filtering to process their results. It is necessary to subtract the background and noise out. After the images are properly processed, the propagation of the shockwaves through the inlet can be tracked. This technique is then used to calculate average Mach number of the shockwaves traveling upstream, given the velocity of the shockwaves. The filtering is also necessary to give better quality visualization of the product backflow.

The technique employed for post-processing the recorded Schlieren data consists of several phases of image processing. The full set of scripts can be found in Appendix B. MATLAB and ImageJ were both used for the processing steps. Using a combination of image subtraction, filtering, and correlation methods [73], each image set is filtered. A subroutine must be applied to the video files to extract each image for individual processing. After each image is cropped to focus on only the flow region, the image of interest is first background subtracted from the first image in the sequence. This is done for every frame in the video file. The first image of the video file acts as a tare image, since there is no presence of detonation or flow in that frame. The image subtraction of the tare frame from each proceeding frame in the video brings all of the flow field into sight. At this point, there is significant noise and unresolved sections of each image. The contrast is adjusted so that dynamic regions are more clearly visible. A Gaussian filter and other spatial image filters are applied. They filter the image based on the intensity of the surrounding regions, in addition to a given threshold and pixel search range. A unique pixel threshold must be assigned for each set of images that are recorded from the different inlet designs. Edge detection methodology was used to assign the shockwave regions a value of 1 , and anything not in the shockwave region a value of 0 . This creates a binary image in which the shockwaves are clearly outlined. However, since automated tracking of the shockwaves was not done, this is not necessary for image processing. The bulk mass of products cannot easily be made into a binary image. Since the region of 
product backflow possesses both pixel intensities of light and dark areas, converting the image to binary would cut part of the products off indefinitely.

The shockwaves can be qualitatively analyzed based on their behavior and shape [74]. In order to extract calibrated, quantitative information about the shockwaves among each inlet test, a lens calibration technique would need to be utilized. However, this was not done for this set of work.

Several factors compounded to make processing the images challenging. First off, since the light source is operated on an AC power supply, there is a low frequency flickering of the light source that can often be seen in the Schlieren videos that is a result of the $60 \mathrm{~Hz}$ AC power source. Secondly, any small particulates in the viewing section (either on the test section windows or in the light path) create noise and disruption in the images. Often, residual material or particles from the print material would remain in the test section and be visible in the images. It was very difficult to get the inlets clean enough to completely eliminate that effect. 


\section{Conclusions}

\section{Chapter 6: Conclusions}

A device for sub-scale testing of RDE inlet designs has been designed and fabricated. This testing device and the work reported here has laid a solid foundation for further development and research into RDE inlets. The testing apparatus allows for interfacing with complex inlet geometries that can be manufactured using a rapid prototype machine. The tests are simple and fast to set up, in comparison to the full scale, hot fired RDE. The testing device provides a method for testing creatively engineered inlet solutions. While there are many possible approaches to the idealized inlet, it seems that conquering the complex gas dynamics that occurs within the RDE and its respective injection geometry is the main task at hand.

The 3D printer used for building each new design was integral throughout this study. Not only did it allow for short turnaround time, but it also produced high-quality parts that were manufactured at high resolutions. It enabled the envelope for design constraints to be pushed far beyond that for machining parts from metal.

While these inlet designs produce promising results towards a more effective inlet design, there are a few key points to indicate. Several of these inlet concepts were designed with a specific operating frequency and wave directionality in mind. However, in experimental testing, these two variables are very inconsistent and unpredictable. Not only are they inconsistent, but they are also difficult to predict. Wave bifurcation is a mysterious phenomenon in RDEs, and will continue to be researched thoroughly. The hope is that this research will lead to a grasp on what causes wave bifurcation, in addition to ways which better control it. This will open the allowable envelope for inlet designs.

Once other hurdles with RDEs are addressed, and the wave frequency and number of detonation waves can be accurately predicted, the injectors should be designed so that it is truncated at the exact length so that the shock reflections from the bottom of the manifolding are colliding with the next set of shockwaves transmitted from the detonation wave. One stark difference between the FSRDE and the LSRDE is that the 


\section{Conclusions}

FSRDE does not have any truncation to its inlet ducting. The shockwave reflections observed in the LSRDE most likely does not occur in the FSRDE, because it has no back surface to reflect off of. Instead it is free to propagate as far upstream into the manifolding until it loses its strength and dissipates. Therefore, changing the manifold so that the air enters perpendicularly will be more conducive to producing the reflected shockwaves off of the bottom surface. This length will need to be tuned to the RDE based on fuel-air mixtures, which will change wave speeds, wave numbers, and hence the propagation characteristics [75]. The shock waves did not seem the have the effect that was hypothesized on the plume of backflow that propagated through the inlet, as was reinforced by [76].

Through this testing platform, there are no measurements in place to understand how each inlet design affects the detonation stability. Also, since no detonation is being sustained through these experiments, it is difficult to estimate the effects that the novel inlets would have on overall combustor performance. For this reason, it is difficult to say which of the three important parameters measured (backflow length, interruption time, and pressure ratios) hold the most importance. Ideally, each of these three metrics could be weighted, so that a design matrix could be created and used to analyze all of the designs in more detail. This would allow each of the design metrics to be weighted, so that each of the design parameters, depending on how critical to combustor performance, would reveal the rank of the designs.

The spade diode, which was the only novel geometry tested for the fuel injectors, performed very well compared to the baseline fuel injectors. It demonstrated significant isolation from the detonation annulus, as well as a reduction in the fuel recovery time. This design should also be adapted to the air injector for testing. Modifications and optimization of the lobe shape, volume, and turning angles for detonation is demanded by the optimistic findings of this work.

The wedge design was effective in reducing backflow lengths. While it is difficult to confidently state that the pressure isolation and interruption times showed any 


\section{Conclusions}

improvement, it is believed that the data returned artifacts that were difficult to avoid. Modifying the wedge shape could yield fruitful results. Running a computational or analytical parametric study on the geometry features is of interest, as this will move towards an optimized ramp shape. The wedge design is a candidate worth executing further testing on.

There are endless possibilities for enhancing the inlet design. It does not have to be limited to down-selecting one chief design either. Multiple designs could be combined to make a more effective and robust design. For instance, the Busemann geometry can be paired with the spade diode. The split path reflector could be combined with the wedges. There are exponential choices to be explored.

The emphasis of this work was to design novel inlet geometries that improve isolation from detonation events. This work is the precursor to reducing the maximum restrictions of an inlet, and reducing the pressure drop. It was successfully shown that some of the novel designs created are successful at reducing the effect of detonation on the inlets refueling capability. The designs with improvement presented here should be explored to prepare for testing in the FSRDE, and measure how the performance changes as a result of the different injection geometry. 


\section{Chapter 7: Recommendations for Future Work}

There is much that is yet to be discovered and tested for RDE inlet studies. Different options for inlet concepts should be investigated, such as active inlet devices (those requiring additional energy to operate). This would include designs such as electromechanical injectors, or very high-speed actuating valves. It may be feasible to pursue using high-speed piezo type injectors in parallel to achieve high frequency cycling. The bottleneck for this approach will most likely be the flow capacity of the valves. An actuator that is able to produce high-frequency and high-amplitude excitations may also serve well in eliminating certain frequency bands. Active control could also consist of some types of spring-loaded check valves farther upstream of the combustor, to avoid damage pressure and temperature levels. Aerodynamic check valves are also a possible option [77]. These kinds of designs are believed to be unrealistic at the present time because of the limitations on MEMS. However, as RDE sizes increase, the operational frequencies will decrease, making it more attainable to control the flow with a MEMS or other mechanical device. This area of technology is continuing to grow, like piezo-ceramics, which will enable ultra-high frequency valve cycling.

While developing an operating, pre-mixed RDE was beyond the focus of this work, it will be beneficial to investigate this more in-depth for the future. Pre-mixing the intake for the RDE will theoretically allow higher combustion efficiency and higher output potential for the combustor, while introducing greater potential risk. Some work on pre-mixed RDEs has been done thus far, but there are many unanswered questions still to address.

Designing an apparatus that allows pressurized flow to be pulsed at a much higher frequency will be a strong step in improving this experimental test rig. Rather than being restricted to a limited number of pulses from the detonation source, it would be better to have an unlimited number of pressure pulses available. A device that could conceivably be adapted for use is one similar to the actuator designed by Beck et al. [78]. Part of this 
change could also be moving the experiment to a lab that has capability for higher flow rates and supply pressures available to the testing rig. Using a PDE to provide the simulated detonation source is possible. Scaling the test rig to accurately reflect RDE conditions for lower PDE frequencies would need to be done.

Attempting to control the inlet flow using a plasma device should also be explored. Dielectric barrier discharge, or DBD, is experiencing increased usage for flow control applications, especially in internal flows for gas turbines [79]. The presence of ionized gases in RDEs makes this an especially viable technique. These devices could be used to energize the flow and alter its flow paths, resulting in better dynamic inlet effects. Magnetohydrodynamics (MHD) has been investigated as a supplementary method of power generation with RDEs, with some promising probable results [80]. However, it could also be leveraged as a method of flow control [81], similar to DBD.

Electrohydrodynamics (EHD), another technology based on plasma actuation, has the same potential that MHD may have.

It will be beneficial to perform Acetone PLIF measurements on the inlet rig while running the detonation source. Hardware has already been connected in place for this diagnostic to be performed. The acetone tank, in addition to the heating tank and all of the lines are currently set up and prepared to run Acetone PLIF measurements. This will allow the inlet backflow to be coupled to the mixing character of each inlet. Measuring mixing of the novel inlets is important, because the interaction between the air and fuel streams changes significantly for some of the new designs. None of the diagnostics executed in this study were sufficient for understanding the change in mixing between fuel and air. The mixing data that was collected computationally in this study was all done without any detonation influence in the channel. This will simulate the combustor more closely to its actual operating conditions. Another diagnostic that would be beneficial to have is accurate velocity measurements within the inlets. Instrumenting with hot wire anemometers would provide accurate, fast-response data for the velocity profiles in the inlet. Its quantitative data would provide confirmation to the Schlieren 
visualization. Flow tagging could be another means to provide more quantitative data for the velocity profiles. The more quantitative data that can be measured from the setup, it will assist in evaluating analytical relations for shockwaves and the aerodynamics of the inlet geometries.

Testing the successful inlet designs discovered in this study in the full-scale, hot fired RDE will be another stepping stone for the inlet improvement program. It will require advanced manufacturing to develop these inlets from a material that can withstand the harsh conditions inside of the RDE. Logical first steps at testing inlet designs in the hot fired RDE may consist of running tests with sectors of a new inlet in place. This will give data about the interaction of the inlet to the detonation, while maintaining safe conditions.

Modifications to this experimental setup can be made that allow it to incorporate design changes for outerbody injection. This would allow designs with symmetry about the detonation channel to be tested. It should also be adapted so that injection can be done from top down into the channel. This will make the testing device more versatile for future designs to be qualified. These modifications lead into testing of more unique inlet designs, like top down injection and injection from the outerbody. Flexibility in fuels and oxidizers for the detonation tube should also be investigated. Firing it on hydrogen and oxygen, as opposed to hydrogen and air, is a worthwhile venture. Interchanging fuel and oxidizer combinations will allow the wave speeds and peak pressures that are achieved to vary.

Other nozzle designs should be investigated for the inlet to be implemented at its choke point. Various nozzles like the plug nozzle, expansion-deflection nozzle, dualthroat nozzle, and a radial flow nozzles are all designs that hold potential. Placing fluidic diodes in a cascade arrangement could also enhance the backflow prevention capability. This has been tested in some other applications [82][83], but would need further development for testing in RDEs. Testing shaped holes for the fuel injectors, like a chevron shaped injector, which is designed to improve mixing potential, is something that 
should be considered for future tests. Adapting other fluidic valves and devices for use as a new inlet geometry should be explored [84].

More work could be invested into transitioning the detonation wave from the detonation tube into the test section. While the detonation inside of the tube is predominantly a planar wave, when it diffuses at the exit of the tube, it becomes more of a cylindrical detonation wave [85]. Rather than a simple expansion of the detonation tube into the combustion channel, shock shapers and other tricks could be used to match the shape and profile to be more similar to that of what propagates in the FSRDE. In fact, with some modifications, a small PDE could even be used to feed the test section its detonation waves.

While it is difficult to gain quantitative data from Schlieren, there are means through which the shockwaves can be reasonably compared between inlet tests. The Schlieren method can be used to gain more quantitative information using a technique such as lens calibration. By taking reference or calibration images of each setup for the unique inlets, the sensitivity and cutoff of the Schlieren system can be calibrated to one another. Any slight changes in the knife edge cut, light positioning, or focus in the Schlieren setup will dramatically change the images that are recorded.

Fabricating an extension for the detonation channel is an important step in eliminating the shock reflections from the detonation exiting the channel. The extension piece should be as long as possible, without interfering in the Schlieren light path. It should also extend the entire height of the test section. This should allow the detonation wave to travel a longer distance while still being constrained. There may still be reflections that travel back upstream, but they should be so weak and damped out by the time the reach the test section that they will be insignificant. This modification will help address the issues that the wedge design was experiencing throughout testing.

As equipment and hardware improves, the diagnostic capability should also improve. With a more robust DAQ unit, the sampling rate can be increased significantly. 


\section{Recommendations for Future Work}

This is a crucial parameter, especially for detonation and shockwave studies. By utilizing a more cutting-edge high-speed camera, better spatial resolution can be obtained for the same frame rate of the current camera. 


\subsection{Lessons Learned}

Many valuable lessons were taken from this study and can be applied towards future work on this inlet design rig. Sealing the inlets inside of the housing proved to be difficult at first. The initial approach was to make gaskets from RTV. However, this not only proved to be messy, but was also not effective at consistently preventing leaks. This led to the improvement of designing O-ring grooves into each side of the inlet geometry, which allowed for small O-rings to be placed in the groove, rather than dealing with RTV seals. The O-rings provided much better sealing around the plenum sections. The small gap between the window edges and the window slots had to be sealed very well also, often with a small bead of RTV.

Another important lesson observed was that it is better to build the fuel and air side injectors as one piece, as opposed to two separate sections. The reason for that is the difficulty in aligning and maintaining the proper placement of the two sections. For the baseline inlet design, it was challenging to accurately maintain the appropriate air gap with the fuel and air sections as two different components. Small shims that were 3D printed seemed to bow and warp, making the air gap difficult to consistently maintain.

Special care was exercised when assembling and disassembling the test rig. The quartz windows proved to be very fragile when installing or removing. The edges of the windows were especially susceptible to cracking or chipping. Once a crack or chip was created, it was only a matter of time until the crack propagated and the window was destroyed. Therefore, extra caution was taken to ensure the window was safely installed each time. Sealing around the window was challenging. Even though the continuous Oring seal did well at preventing the majority of the leakage, small amounts of air and helium were able to slip between the window edges and the aluminum sidewalls. This was counteracted with an RTV seal that was pushed into the crack as much as possible. A secondary approach to combat the leakage was adding supports in the inlet section that incorporated an O-ring seal as well. This concept is shown in Figure 56. In future designs 
and tests, making the window large enough that the O-ring seals only on the window, not the aluminum, will be a much better design solution.

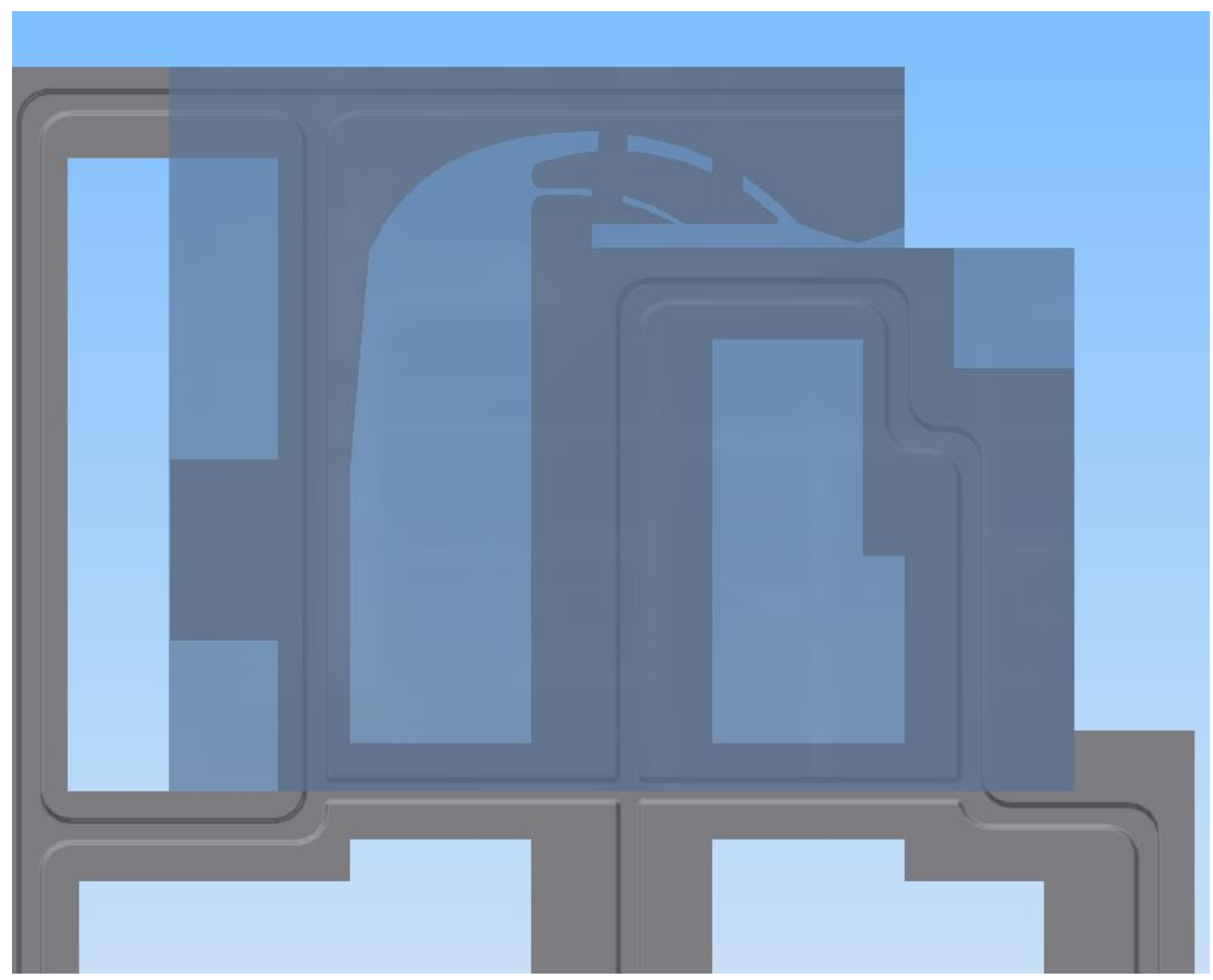

Figure 56. Sealing design change to reduce leakage around window

It was determined early in testing that products of detonation were exiting the channel and lingering in the imaging section of the Schlieren setup long after the test detonations had passed by. This caused gradients of the detonation byproducts to be imaged long after the detonation waves had passed through the inlet sections. The issue is clearly seen in Figure 57, and was only observed for the 0.022 and 0.044 inch gap design. To help remedy this issue, an extension was made for the channel so that the products would get carried far enough out and away from the imaging section so that it was no 
longer in the light path. Ensuring a good seal on the upstream portion of the test section, where the detonation tube enters the channel, was important in remedying this issue. The channel extension is another modification that helped address that. This issue was resolved after these specific modifications were made, and it had no direct impact on the measurements that were made and reported.

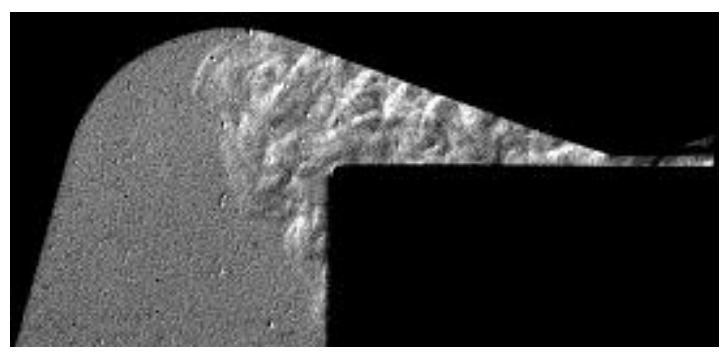

Figure 57. Detonation byproducts imaged long after detonations extinguish 


\section{References}

[1] Lefebvre, Arthur H., and Dilip R. Ballal. Gas Turbine Combustion: Alternative Fuels and Emissions. Boca Raton: Taylor \& Francis, 2010.

[2] Nordeen, C.A., "Thermodynamics of a Rotating Detonation Engine," PhD dissertation, University of Connecticut, 2013.

[3] Langston, Lee S., "PALS - An Auspicious New Gas Turbine Seal." Mechanical Engineering. March 2016. pp 54-55.

[4] Frolov, S.M., Dubrovskii, A.V., Ivanov, V.S., "Three-Dimensional Numerical Simulation of the Operation of the Rotating-Detonation Chamber." Combustion, Explosion, and Shock Waves. Vol. 31, No. 3, pp 32-45.

[5] Aerodynamics Research Center. "Pulsed Detonation Engines." The Universtiy of Texas at Arlington. College of Engineering Graduate School, Mechanical and Aerospace Engineering. 2014.

[6] Rich, Ben R., and Janos, Leo. Skunk Works: A Personal Memoir of My Years at Lockheed. Boston: Little, Brown, 1994.

[7] Wolanski, P. "Detonative Propulsion.” Proceedings of the Combustion Institute 34 (2013). pp 125-158.

[8] Richards, G., "New Developments in Combustion Technology, Part II: Step change in efficiency." 2012 Princeton, CEFRC Summer School in Combustion.

[9] Swamy, Y.M., "Parametric Analysis of a Detonation-Type Turbofan." MS Thesis, University of Texas at Arlington. December 2011.

[10] Miri, S.H., “Shock-Less Hypersonic Intakes.” MS Thesis. Ryerson University. Ontario, Canada, January 2012.

[11] Naples, A., Hoke, J., Karnesky, J., Schauer, F., "Flowfield Characterization of a Rotating Detonation Engine." $51^{\text {st }}$ AIAA Aerospace Sciences Meeting including the New Horizons Forum and Aerospace Exposition. AIAA 2013-1178. 7 - 10 January, 2013. 
[12] Ma, H., Wu, X.S., Wang, D., Cai, W.X., Chen, J., Diao, J.Y., Chen-xi, P., "Experimental Investigation of a Reed Valve on the Performance of a Pulse Detonation Engine". International Journal of Turbo Jet-Engines. 2013.

[13] Lisanti, J.C., Roberts, W.L., "Design of an Actively Valved and Acoustically Resonant Pulse Combustor for Pressure-gain Combustion Applications.” AIAA SciTech. 4-8 January 2016. San Diego, CA. AIAA 2016-0899.

[14] Thorpe, E., "Design, Fabrication, and Testing of a Multi-Cycle Pulse Detonation Engine," MS Thesis, West Virginia University. December 2015.

[15] Lu, F.K., Jensen, D.S., "Potential Viability of a Fast-Acting Micro-Solenoid Valve for Pulsed Detonation Fuel Injection.” AIAA 2003-0888.

[16] Meyers, J.M., Lu, F.K., Wilson, D.R., "Performance Enhancements on a Pulsed Detonation Engine." AIAA 2003-1173.

[17] Fotia, M. L., Schauer, F., Kaemming, T., Hoke, J., "Study of the Experimental Performance of a Rotating Detonation Engine with Nozzled Exhaust Flow.” AIAA SciTech. AIAA 2015-0631. 5 - 9 January 2015.

[18] Glassman, Irvin, and Richard A. Yetter. Combustion. Amsterdam: Elsevier, 2008.

[19] Lu, F.K., Braun, E.M., Massa, L., Wilson, D. R., "Rotating Detonation Wave Propulsion: Experimental Challenges, Modeling, and Engine Concepts (Invited)." $47^{\text {th }}$ AIAA/ASME/SAE/ASEE Joint Propulsion Conference \& Exhibit. 31 July - 3 August 2011. San Diego, CA.

[20] Forster, F.K., Bardell, R.L., Afromowitz, M.A., Sharma, N.R., Blanchard, A., "Design, Fabrication and Testing of Fixed-Valve Micro-Pumps". Proceedings of the ASME Fluids Engineering Division. IMECE. 12 - 17 November, 1995.

[21] Cocks, P.A.T., Holley, A.T., Rankin, B.A., "High Fidelity Simulations of a NonPremixed Rotating Detonation Engine.” AIAA SciTech. 4 - 8 January 2016. San Diego, CA.

[22] Mundt, I.C., Lieser, I.J., "Performance Improvement of Propulsion Systems by Optimization of the Mixing Efficiency and Pressure Loss of Forced Mixers." $8^{\text {th }}$ European Propulsion Forum. 26 - 28 March 2001, Nottingham. 
[23] Weston, Kenneth C., Energy Conversion. Brooks/Cole Publishing, 1992.

[24] Lim, W.H.E, "Gas dynamic Inlet Isolation in Rotating Detonation Engine." MS Thesis, Naval Postgraduate School. December 2010.

[25] Doster, J.C., "Hypermixer Pylon Fuel Injection for Scramjet Combustors." Air Force Institute of Technology, PhD. Dissertation. September 2008.

[26] Schwer, D.A., Kailasanath, K., "On Reducing Feedback Pressure in Rotating Detonation Engines". $51^{\text {st }}$ AIAA Aerospace Sciences Meeting including the New Horizons Forum and Aerospace Exposition. AIAA 2013-1178. 7 - 10 January, 2013.

[27] Schwer, D.A., Kailasanath, K., "Effect of Inlet on Fill Region and Performance of RDE", Joint Propulsion Conference. July 31 - August 3, 2011. San Diego, California.

[28] Fotia, M.L., Hoke, J.L., Schauer, F. "Propellant Plenum Dynamics in a Two-Dimensional Rotating Detonation Experiment.” AIAA SciTech. AIAA 2014-1013. 13 - 17 January 2014.

[29] Naples, A., Hoke, J.L., Schauer, F.R., "Experimental Investigation of a Rotating Detonation Engine Fuel Injector Temporal Response." AIAA SciTech. AIAA 2015-1104. 5 - 9 January 2015.

[30] Anand, V., St. George, A., Driscoll, R., Gutmark, E., "Analysis of air inlet and fuel plenum behavior in a rotating detonation combustor." Experimental Thermal and Fluid Science. Volume 7. January 2016, pp 408-416.

[31] Braun, E.M., Dunn, N. L., Lu, F.K., "Testing of a Continuous Detonation Wave Engine with Swirled Injection." $48^{\text {th }}$ AIAA Aerospace Sciences Meeting Including the New Horizons Forum and Aerospace Exposition. AIAA 2010-146. 4 - 7 January, 2010. Orlando, Florida.

[32] Braun, E.M., Balcazar, T.S., Wilson, D.R., Lu, F.K., "Experimental Study of a HighFrequency Fluidic Valve Fuel Injector.” AIAA Joint Propulsion Conference. AIAA 20115545. 31 July - 3 August 2011.

[33] Peace, J.T., Joshi, D.D., Lu, F.K., "Experimental Study of High-Frequency Fluidic Valve Injectors for Detonation Engine Applications.” AIAA SciTech 2014. AIAA 2014-1318. 
[34] Drummond, J. P., Diskin, G.S., Cutler, A.D., "Chapter 6: Fuel-Air Mixing and Combustion in Scramjets." Technologies for Propelled Hypersonic Flight. North Atlantic Treaty Organization, Research and Technology Organization. January 2006.

[35] Driscoll, R., St. George, A., Anand, Vijay, Randall, S., Gutmark, E.J., "Numerical Investigation of Inlet Injection in a Rotating Detonation Engine.” AIAA SciTech. AIAA 2015-0879. 5 - 9 January 2015.

[36] Shank, J.C., "Development and Testing of a Rotating Detonation Engine Run on Hydrogen and Air", MS Thesis, AFIT/GAE/ENY/12-M36, Air Force Institute of Technology, WPAFB, Ohio, March 2012.

[37] Hariharan, A.R., Babu, V., "Transverse Injection into a Supersonic Cross Flow through a Circular Injector with Chevrons.” ASME Journal of Fluids Engineering. Volume 136, 021204-1. February 2014.

[38] Russo, R.M., “Operational Characteristics of a Rotating Detonation Engine Using Hydrogen and Air." MS Thesis, AFIT/GAE/ENY/11-J03, Air Force Institute of Technology, WPAFB, Ohio, June 2011.

[39] Bobusch, B.C., "Fluidic Devices for Realizing the Shockless Explosion Combustion Process.” Technischen Universität Berlin, PhD. Dissertation. January 21, 2015.

[40] Andrus, I.Q., King, P.I., Fotia, M.L., Schauer, F.R., Hoke, J.L., “Experimental Analogue of a Pre-Mixed Rotating Detonation Engine in Plane Flow.” AIAA SciTech. AIAA 20151105. 5 - 9 January 2015.

[41] Barakauskas, E.J., "Sudden expansion of a Bounded Jet at High Pressure Ratio.” AIAA Journal, Vol. 2, No. 9. September 1964, pp 1644-1645.

[42] Andrus, I.Q., King, P.I., Polanka, M.D., Schauer, F.R., Hoke, J.L., "Design of a Premixed Fuel-Oxidizer System to Prevent Flashback in a Rotating Detonation Engine." AIAA SciTech. 4 - 8 January 2016. San Diego, CA.

[43] Burr, J., Yu, K. H., "Detonation Reignition within a Rotating Detonation Engine.” AIAA SciTech. 4 - 8 January 2016. San Diego, CA.

[44] Turquis, M. Pascal. "Process for Controlling the Valves of an Internal Combustion Engine.” Peugeot Citroen Automobiles Sa, assignee. Patent US6981475 B2. 3 Jan. 2006. 
[45] Santana, A., Silva, M.S., Lacava, P.T., Goes, L.C.S., “Acoustic Cavities Design Procedures." Thermal Engineering, Vol. 6, No 2. December 2007.

[46] Paxson, D.E., "Impact of an Exhaust Throat on Semi-Idealized Rotating Detonation Engine Performance.” AIAA SciTech. 4 - 8 January 2016. San Diego, CA.

[47] Fujiwara, T., Hishida, M., Kindracki, J., Wolanski, P., "Stablization of Detonation for Any Incoming Mach Numbers.” Combustion, Explosion, and Shock Waves, Vol. 45, No. 5, pp. 603-605, 2009.

[48] Rankin, B.A., Fotia, M.L., Paxson, D.E., Hoke, J.L., Schauer, F.R., "Experimental and Numerical Evaluation of Pressure Gain Combustion in a Rotating Detonation Engine." AIAA SciTech. AIAA 2015-0877. 5 - 9 January 2015.

[49] Bedick, C., Sisler, A., Ferguson, D., Strakey, P., "Development of a Lab-Scale Experimental Testing Platform for Rotating Detonation Engine Inlets" AIAA SciTech, January 2017.

[50] Munson, Bruce Roy, T.H. Okiishi, Wade W. Huebsch, and Alric P. Rothmayer. Fundamentals of Fluid Mechanics. Hoboken, NJ: John Wiley \& Sons, 2013.

[51] Settles, G.S. Schlieren and Shadowgraph Techniques: Visualizing Phenomena in Transparent Media. Berlin: Springer, 2001.

[52] Goulding, J.S., “A Study of Large-Scale Focusing Schlieren Systems.” Research Report. University of Witwatersrand, Johannesburg, 2006.

[53] Strakey, P.A., Ferguson, D.H., Sisler, A.T., Nix, A.C., “Computationally Quantifying Loss Mechanisms in a Rotating Detonation Engine.” AIAA SciTech 2016. 4 - 8 January 2016. San Diego, CA.

[54] Oh, J. Y., Ma, F., Hsieh, S.Y., Yang, V., "Interactions between Shock and Acoustic Waves in a Supersonic Inlet Diffuser." Journal of Propulsion and Power, Vol. 21, No. 3. May-June 2005.

[55] Robertson, Scott. “The Piston Made of Air.” Pneumatic Options Research Library. $4^{\text {th }}$ Edition.

[56] Turquis, M. Pascal. Process for Controlling the Valves of an Internal Combustion Engine. Peugeot Citroen Automobiles, assignee. Patent US6981475 B2. 3 Jan 2006. 
[57] Fuller, R.P., Wu, P.K., Nejad, A.S., Schetz, J.A., “Comparison of Physical and Aerodynamic Ramps as Fuel Injectors in Supersonic Flow" Journal of Propulsion and Power, Vol. 14, No 2. April 1998.

[58] Panicker, P.K., "The Development and Testing of Pulsed Detonation Engine Ground Demonstrations." PhD Dissertation. The University of Texas at Arlington. August 2008.

[59] Gamezo, V.M., Khokhlov, A.M., Oran, E.S., "The Influence of Shock Bifurcations on Shock-Flame Interactions and DDT.” Combustion and Flame, 126 (4), 1810-1826.

[60] Edwards, D.H., Thomas, G.O., Nettleton, M.A., “The Diffraction of a Planar Detonation Wave at an Abrupt Area Change.” Journal of Fluid Mechanics, vol. 95, part 1. pp 79-96.

[61] Bykovskii, Fedor, A., Sergey A. Zhdan, and Evgenii F. Vedernikov. "Continuous Spin Detonations," Journal of Propulsion and Power, 22: 1204-1216 (2006).

[62] Hopper, D.R., King, P. I., Schauer, F.R., "Propagation of Detonations across a Step Area Change in a Pulsed Detonation Engine." $45^{\text {th }}$ AIAA Aerospace Sciences Meeting and Exhibit. Reno, NV. 8 - 11 January 2007.

[63] Huh, H., Kim, J., Driscoll, J.F., "Measured Characteristics of Flow and Combustion in Supersonic Flame/Shock Wave Interaction.” $37^{\text {th }}$ AIAA/ASME/SAE/ASEE Joint Propulsion Conference. Salt Lake City, UT. 8 - 11 July, 2001.

[64] Délery, Jean. Handbook of Compressible Aerodynamics. Hoboken: Wiley, 2010.

[65] Buckmaster, J., Jackson, T.L., Kumar, A., Combustion in High-Speed Flows. Volume 1. Norwell, MA: Kluwer Academic, 1994.

[66] Matsushima, K., Maruyama, D., Kusunose, K., Noguchi, R., "Extension of Busemann Biplane Theory to Three Dimensional Wing Fuselage Configurations." $27^{\text {th }}$ International Congress of the Aeronautical Sciences, 2010.

[67] Yamashita, H. Kuratani, N., Yonezawa, M., Ogawa, T., Nagai, H., Asai, K., Obayashi, S., "Wind Tunnel Testing on Start/Unstart Characteristics of Finite Supersonic Biplane Wing.” International Journal of Aerospace Engineering. Volume 2013, ID 231434.

[68] Mohsen, A.M., Yusoff, M.Z., Al-Falahi, A., Shuarib, N.H., "The Effects of Area Contraction on Shock Wave Strength and Peak Pressure in Shock Tube." International Journal of Automotive and Mechanical Engineering. Vol. 5, pp 587-596. 2012. 
[69] Lewis, Bernard, and Guenther Von Elbe. Combustion, Flames, and Explosions of Gases. Orlando: Academic, 1987.

[70] Kinsler, Lawrence E., Austin R. Frey, Alan B. Coppens, and James V. Sanders. Fundamentals of Acoustics. Third ed. New York: Wiley, 1982.

[71] Valvular Conduit. Nikola Tesla, assignee. Paten US 1329559 A. 3 Feb. 1920.

[72] Ben-Dor, Gabi, Ozer Igra, Tov Elperin, and Assa Lifshitz. Handbook of Shock Waves. San Diego: Academic, 2001.

[73] Hargather, M.J., "Background-Oriented Schlieren Diagnostics for Large-Scale Explosive Testing.” Shock Waves Journal, Springer-Verlag Berlin Heidelberg, 18 April 2013.

[74] Ben-Dor, Gabi. Shock Wave Reflection Phenomena. Berlin: Springer, 2007.

[75] Joshi, Narendra Digamber, and Michael Solomon Idelchik. Tuned Cavity Rotating Detonation Combustion System. General Electric Company, assignee. Patent EP 2884184 A1. 17 June 2015.

[76] Kwatra, N., Greatarsson, J.T., Fedkiw, R., "Practical Animation of Compressible Flow for Shock Waves and Related Phenomena.” Eurographics Symposium on Computer Animation, 2010.

[77] Linderoth, E. Torvald. “Aerodynamic Check Valve.” Patent US2727535. 20 December 1955.

[78] Beck, B.T., Cutler, A.D., Drummond, J.P., Jones, S.B. “A Resonant Pulse Detonation Actuator for High-Speed Boundary Layer Separation Control." $11^{\text {th }}$ International Symposium of Flow Visualization. 9 - 12 August, 2004. Notre Dame, Indiana.

[79] Ashpis, D.E., Thurman, D.R., "Test Conditions for Performance Characterization of Dielectric Barrier Discharge (DBD) Plasma Actuators for Active Flow Control in Jet Engines.” Proceedings of ASME Turbo Expo 2011. GT 2011-46583. June, 2011. Vancouver, Canada.

[80] Braun, J., Saracoglu, B.H., Magin, T.E., Paniagua, G., "Mono-dimensional analysis of the MagnetoHydrodynamic effect in Rotating Detonation Combustors." AIAA SciTech. 4 - 8 January 2016. San Diego, CA. AIAA 2016-0898.

[81] Gad-el-Hak, Mohamed. Flow Control: Passive, Active, and Reactive Flow Management. Cambridge: Cambridge UP, 2000. 
[82] Okuhara, S., Takao, M., Sato, H., Takami, A., Setoguchi, T., “A Twin Unidirectional Impulse Turbine for Wave Energy Conversion-Effect of Fluidic Diode on the Performance.” Open Journal of Fluid Dynamics 4, 2014. pp 433-439.

[83] Gokoglu, S.A., Kuczmarski, M.A., Culley, D.E., Raghu, S., "Numerical Studies of a Supersonic Fluidic Diverter Actuator for Flow Control." $5^{\text {th }}$ Flow Control Conference. Chicago, IL., June 28-July 1, 2010. AIAA 2010-4415.

[84] Kirshner, Joseph M., and Silas Katz. Design Theory of Fluidic Components. New York: Academic, 1975.

[85] Wakita, M., Tamura, M., Terasaka, A., Sajiki, K., Totani, T., Nagata, H., “Detonation Transition around Cylindrical Reflector of Pulse Detonation Engine Initiator.” Journal of Propulsion and Power. Vol. 29, No. 4. July-August 2013. 


\section{Appendix A: Data Processing \& Design Calculations \\ MATLAB}

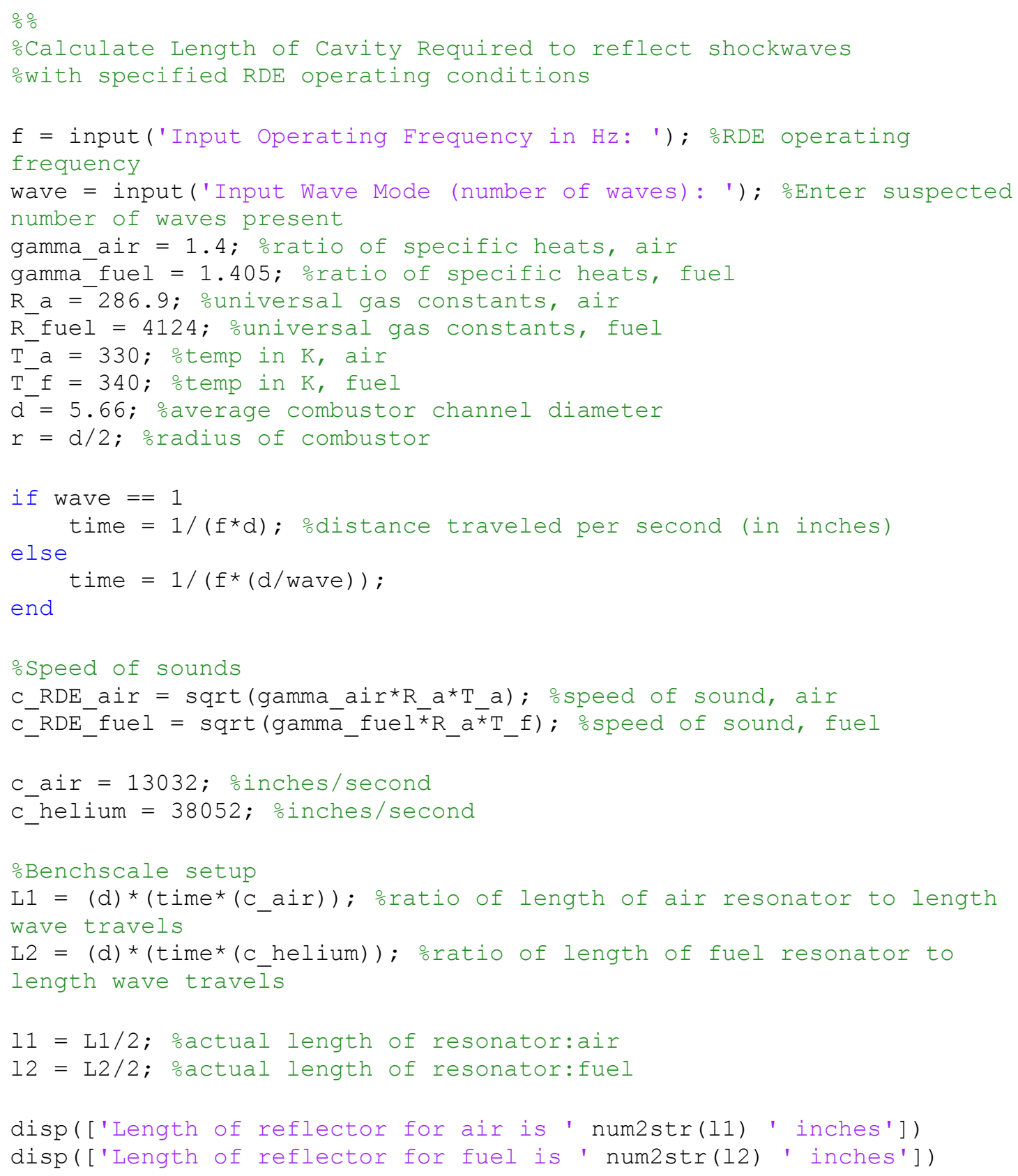




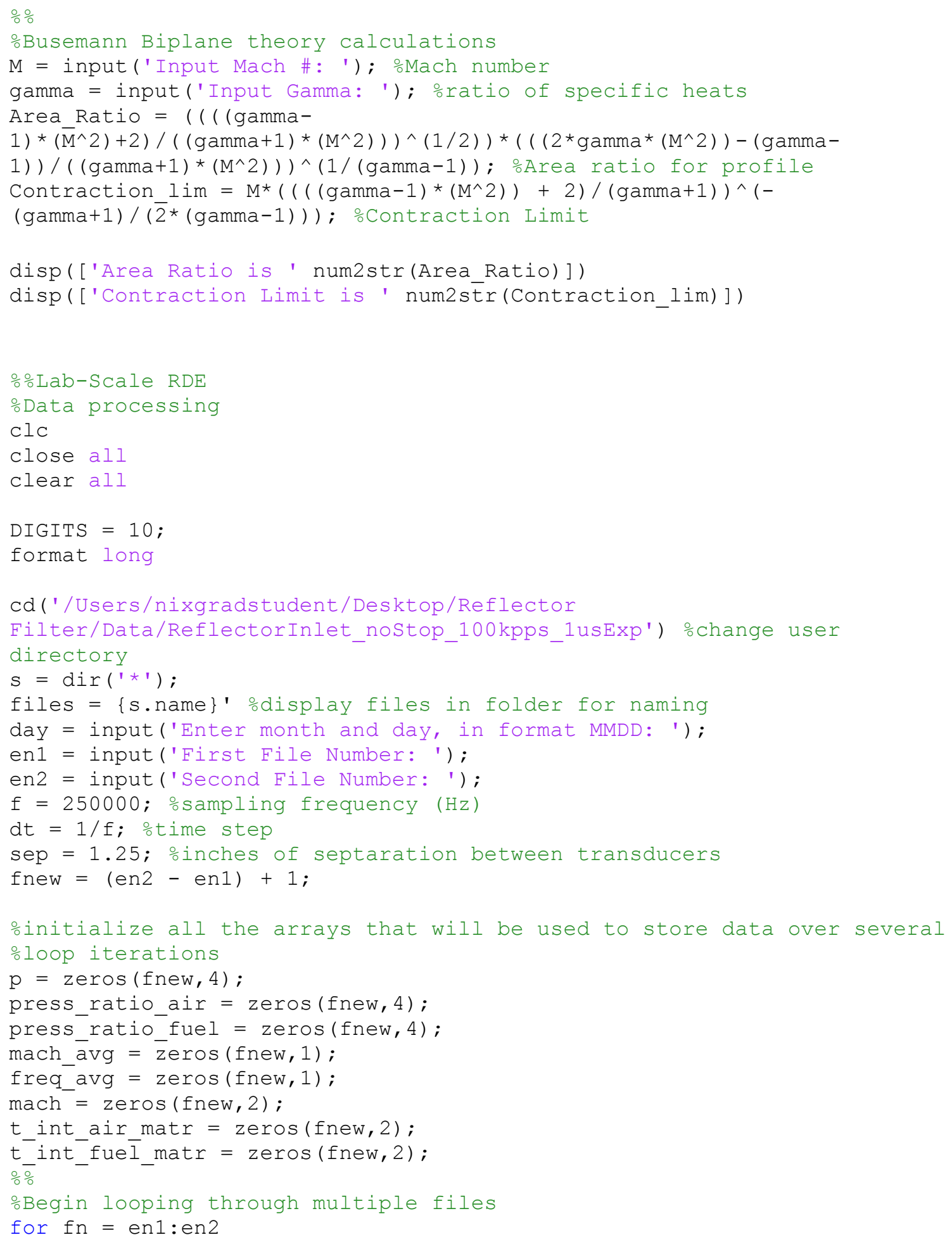




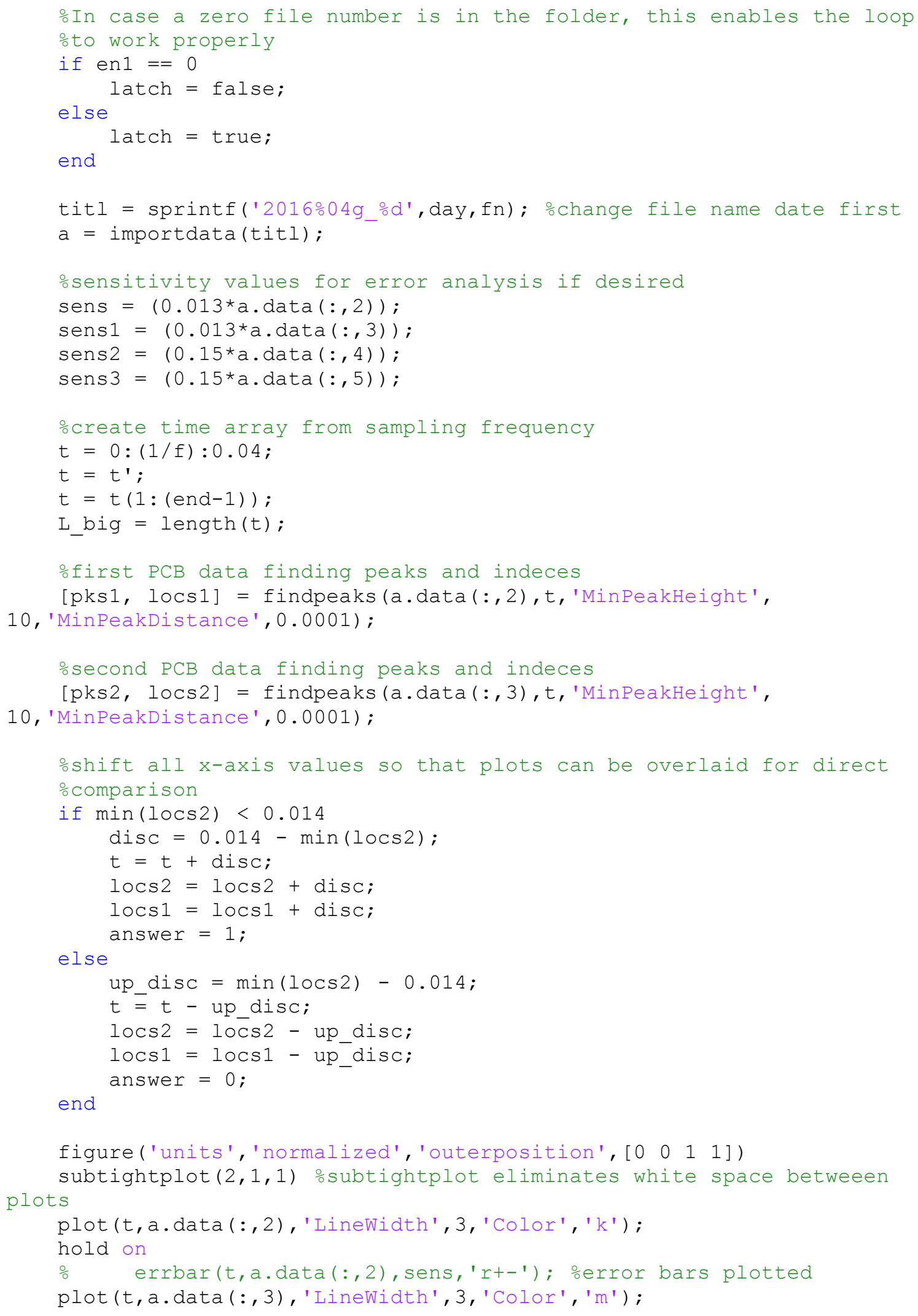




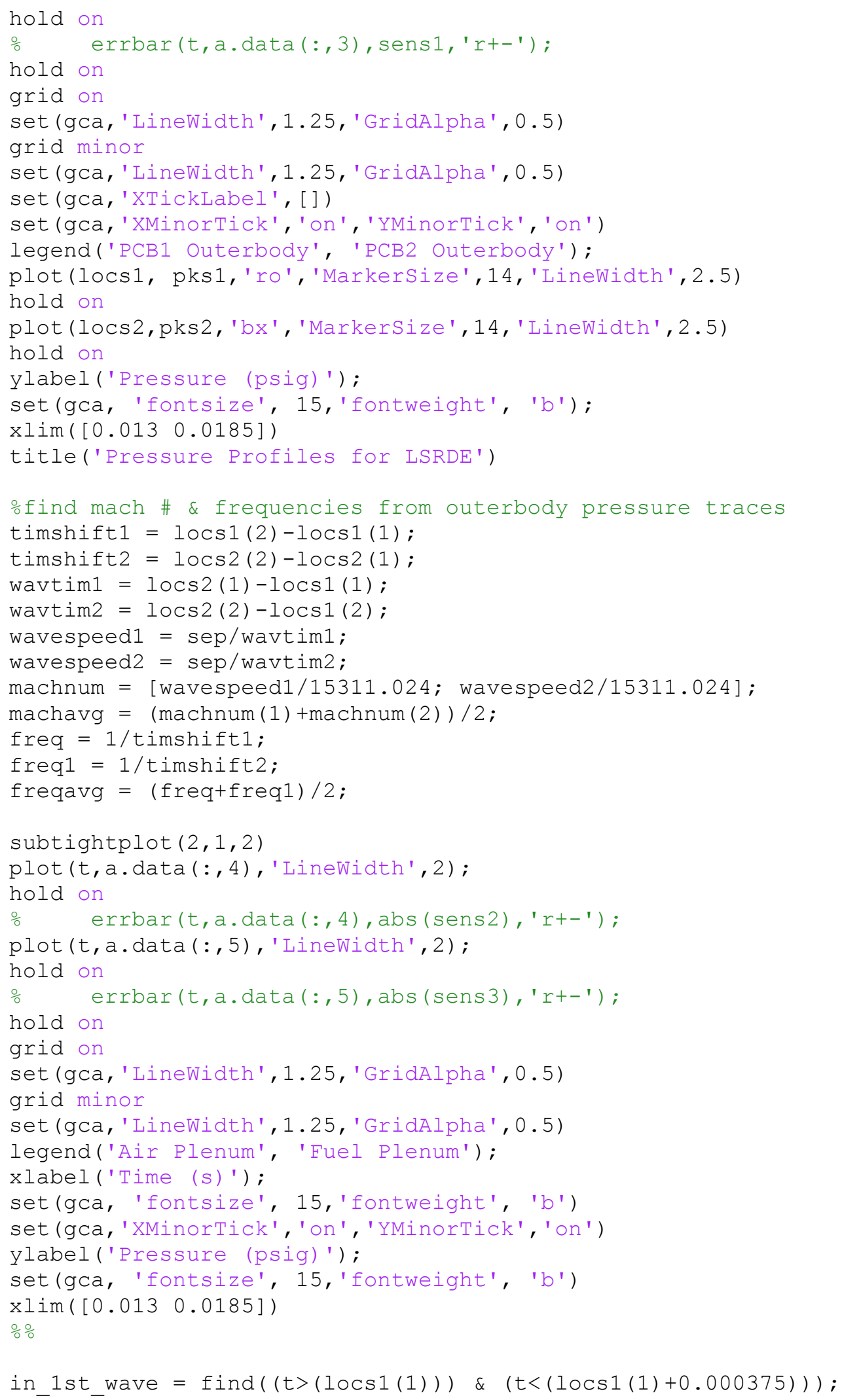




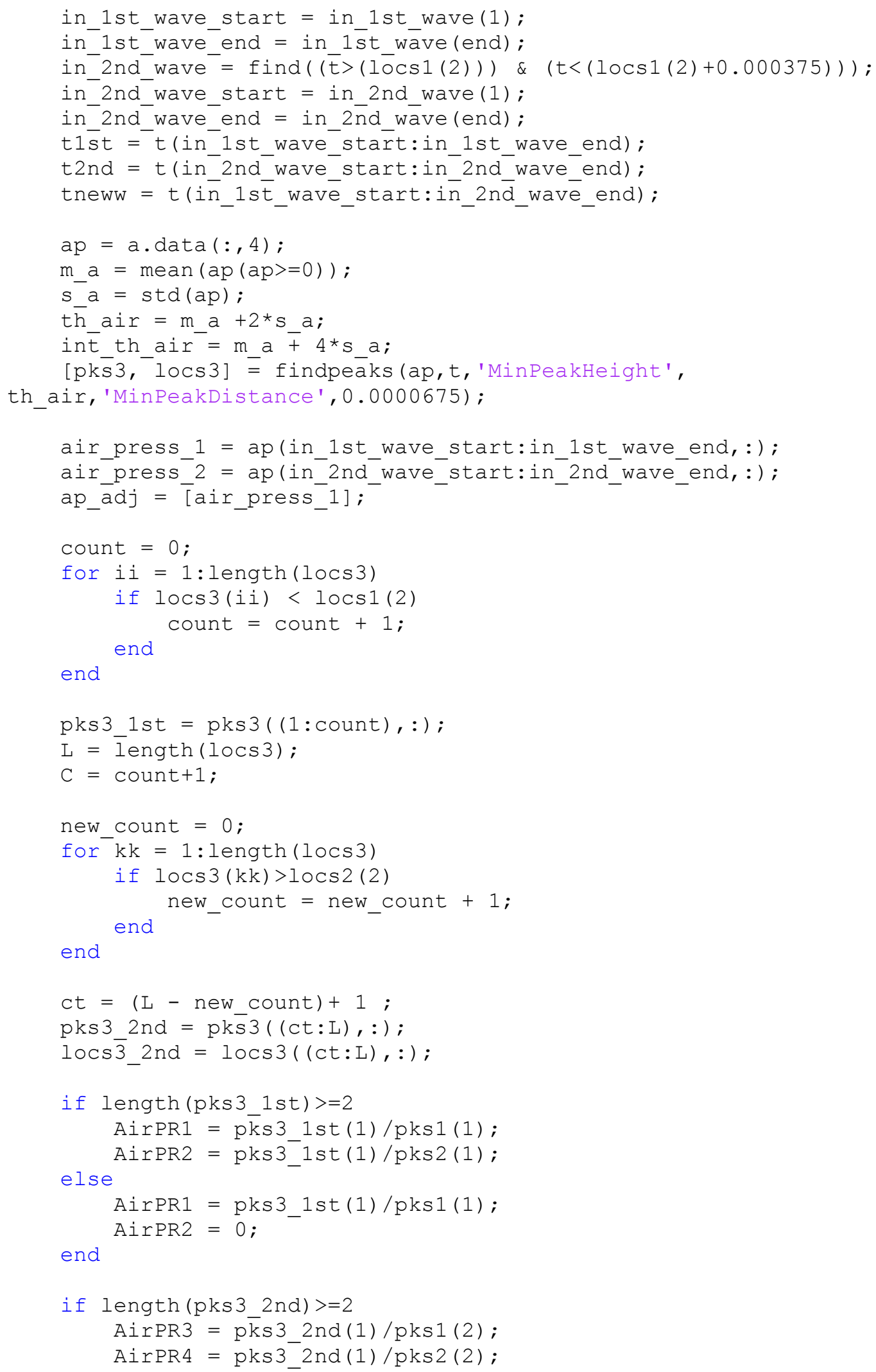




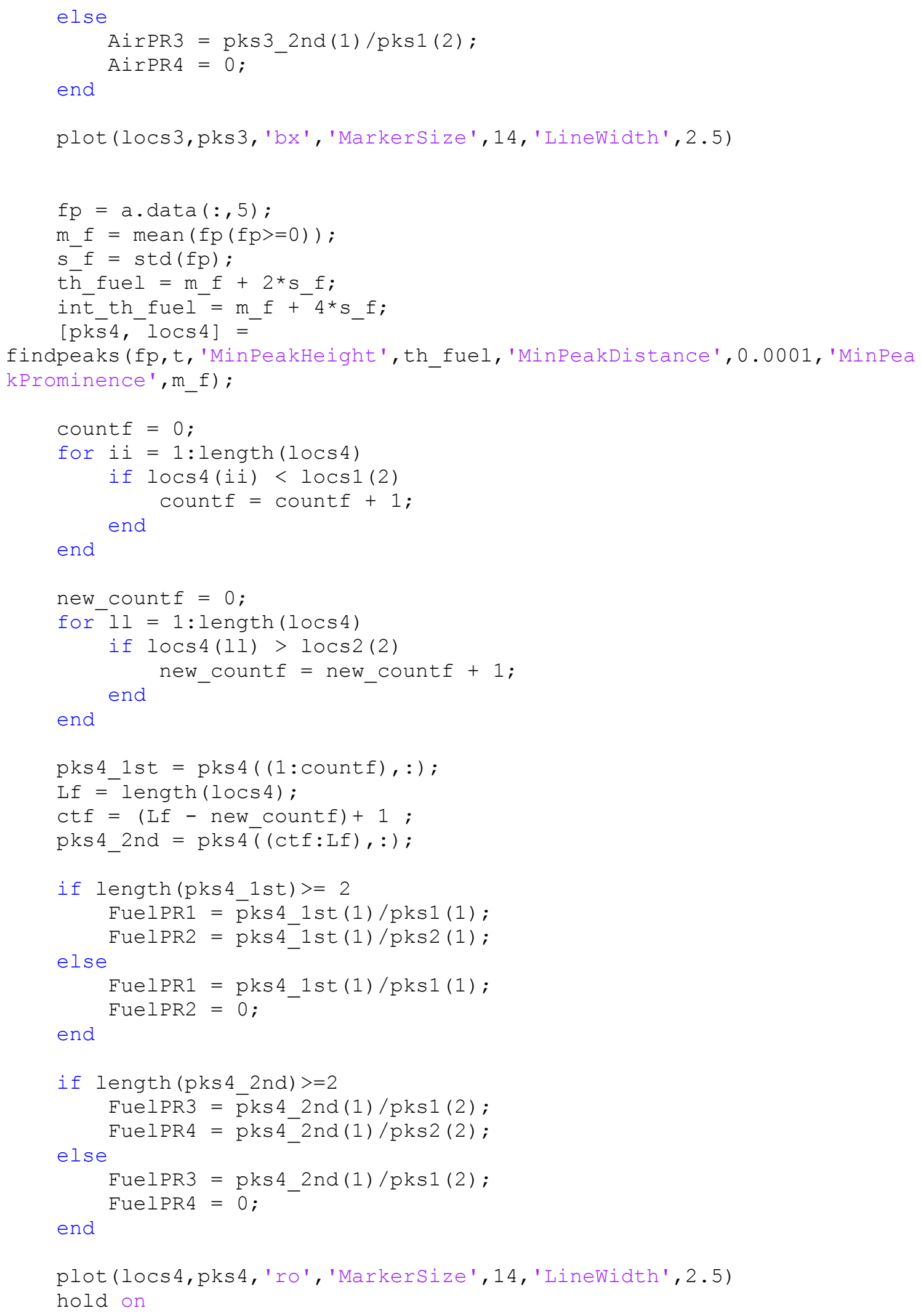




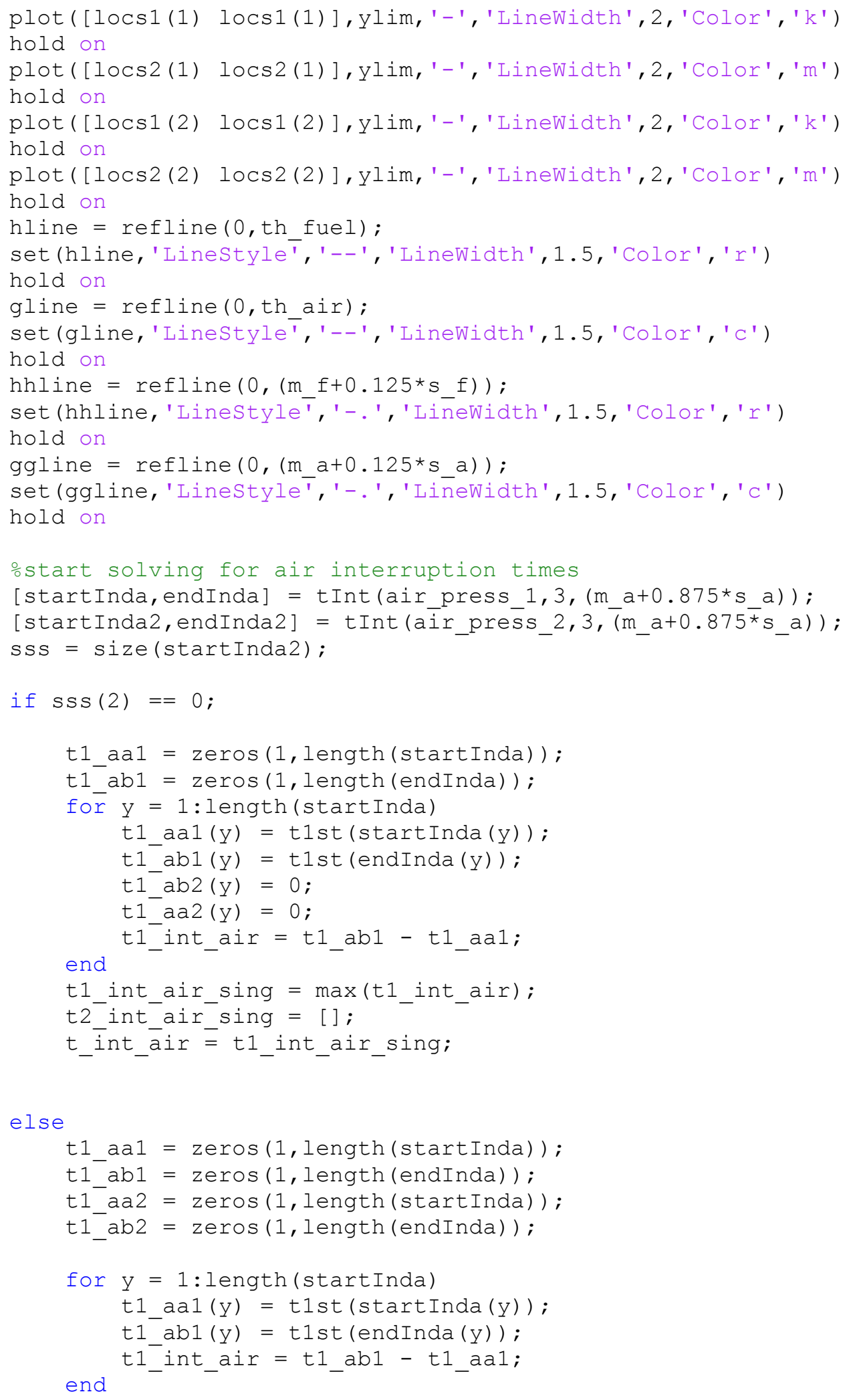




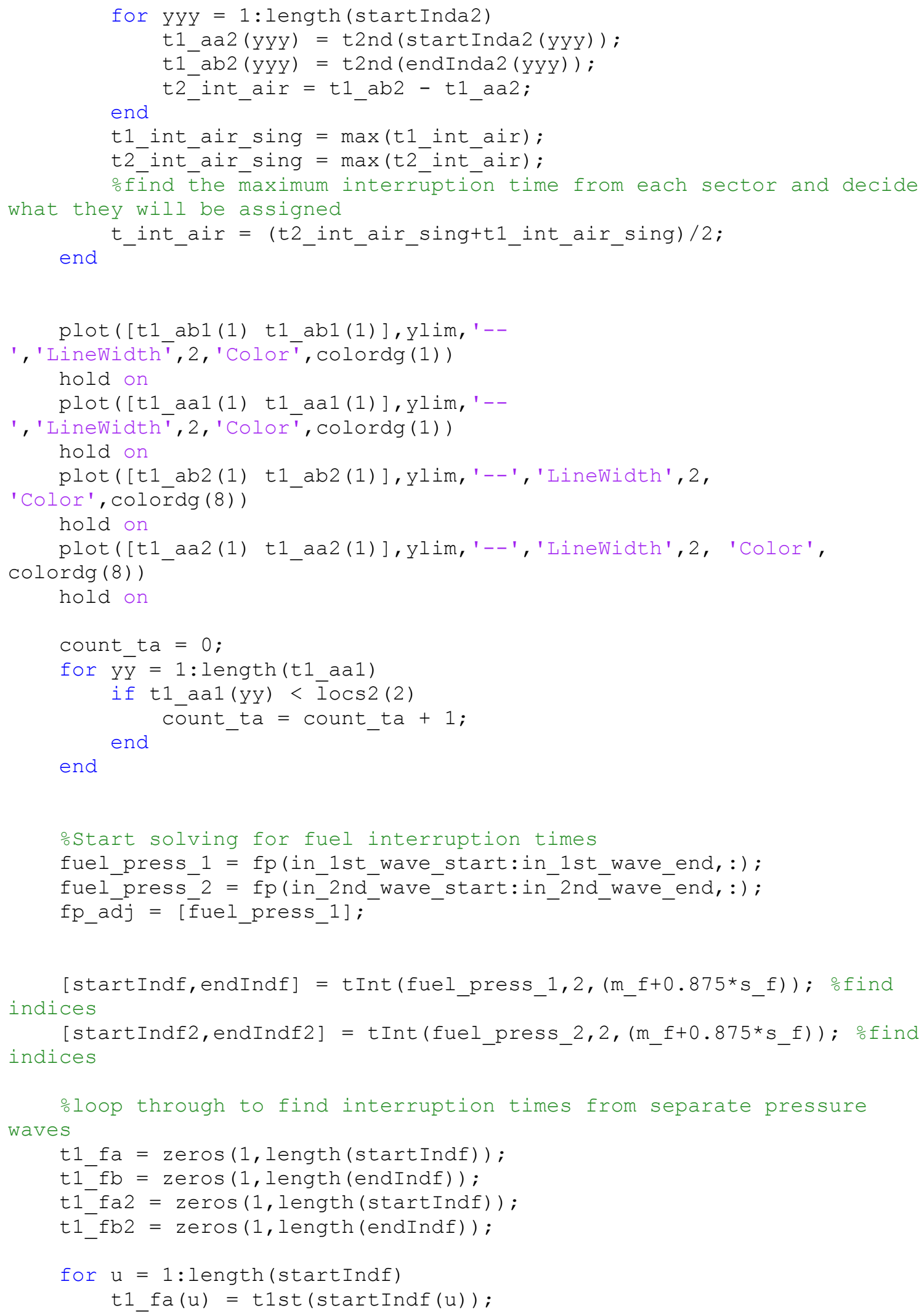




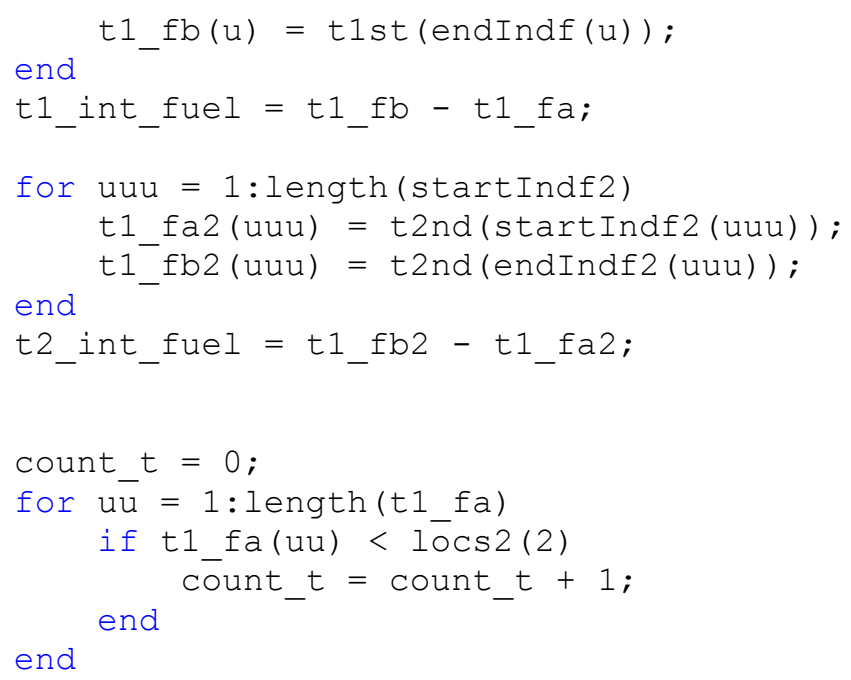




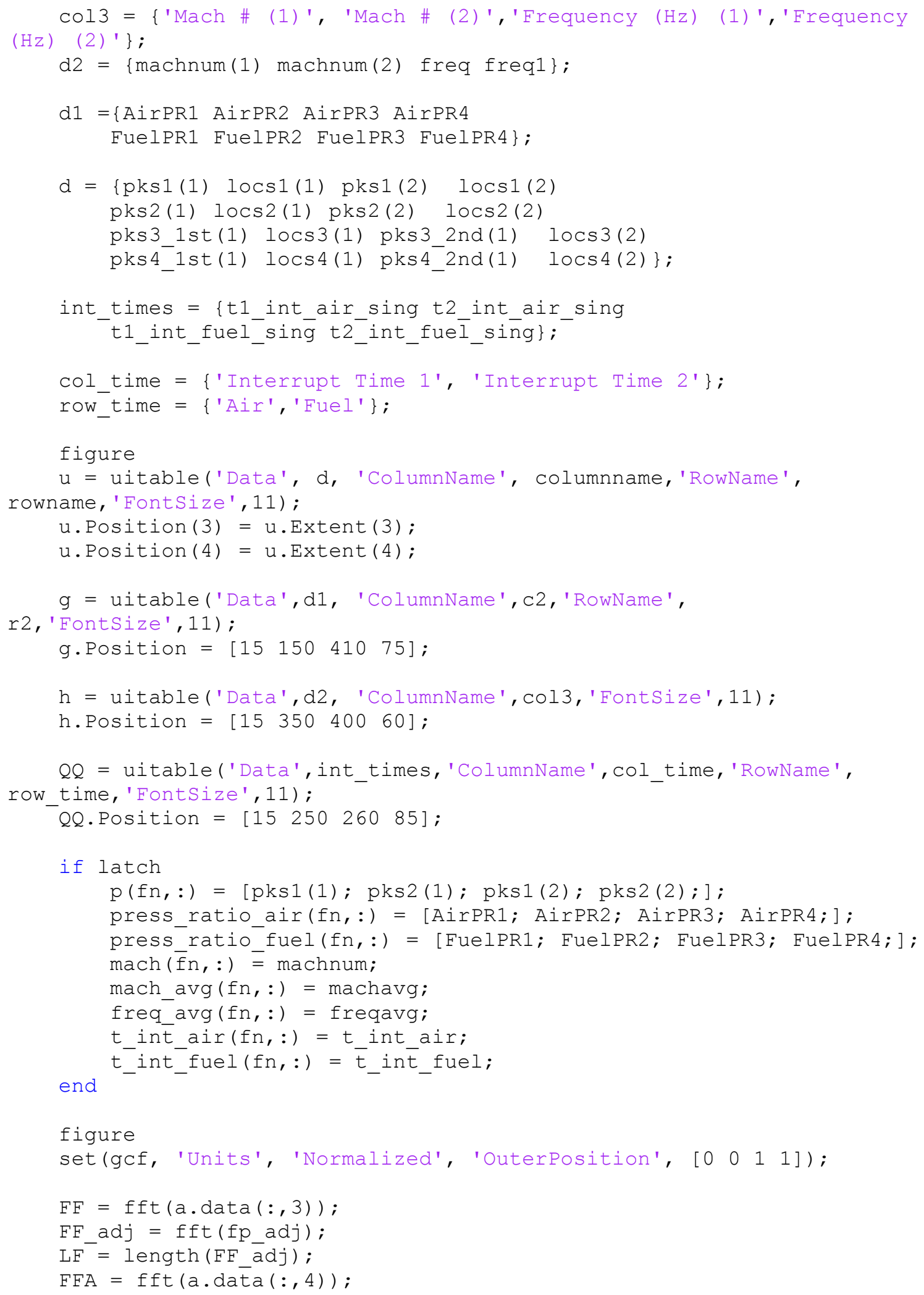




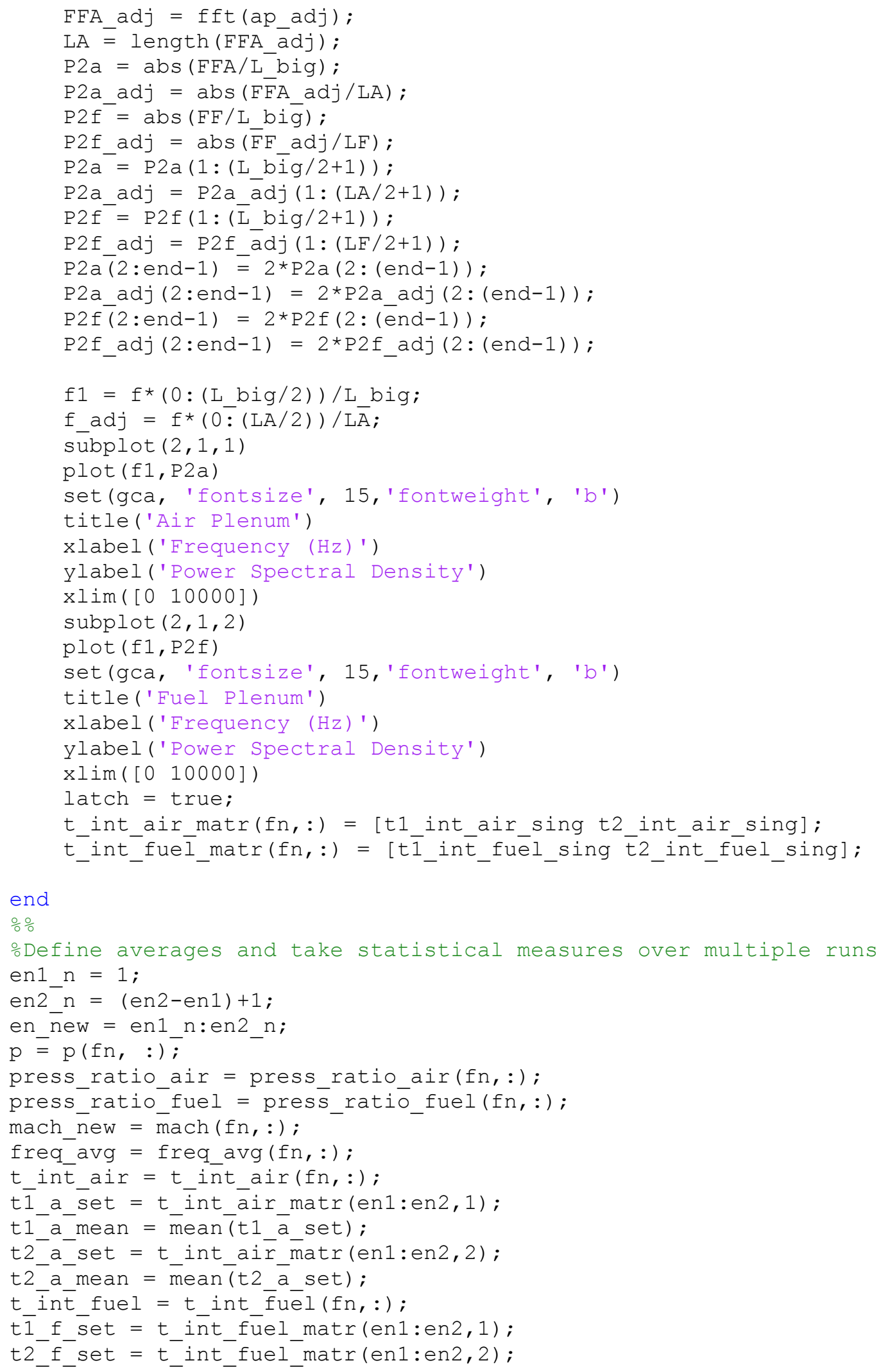




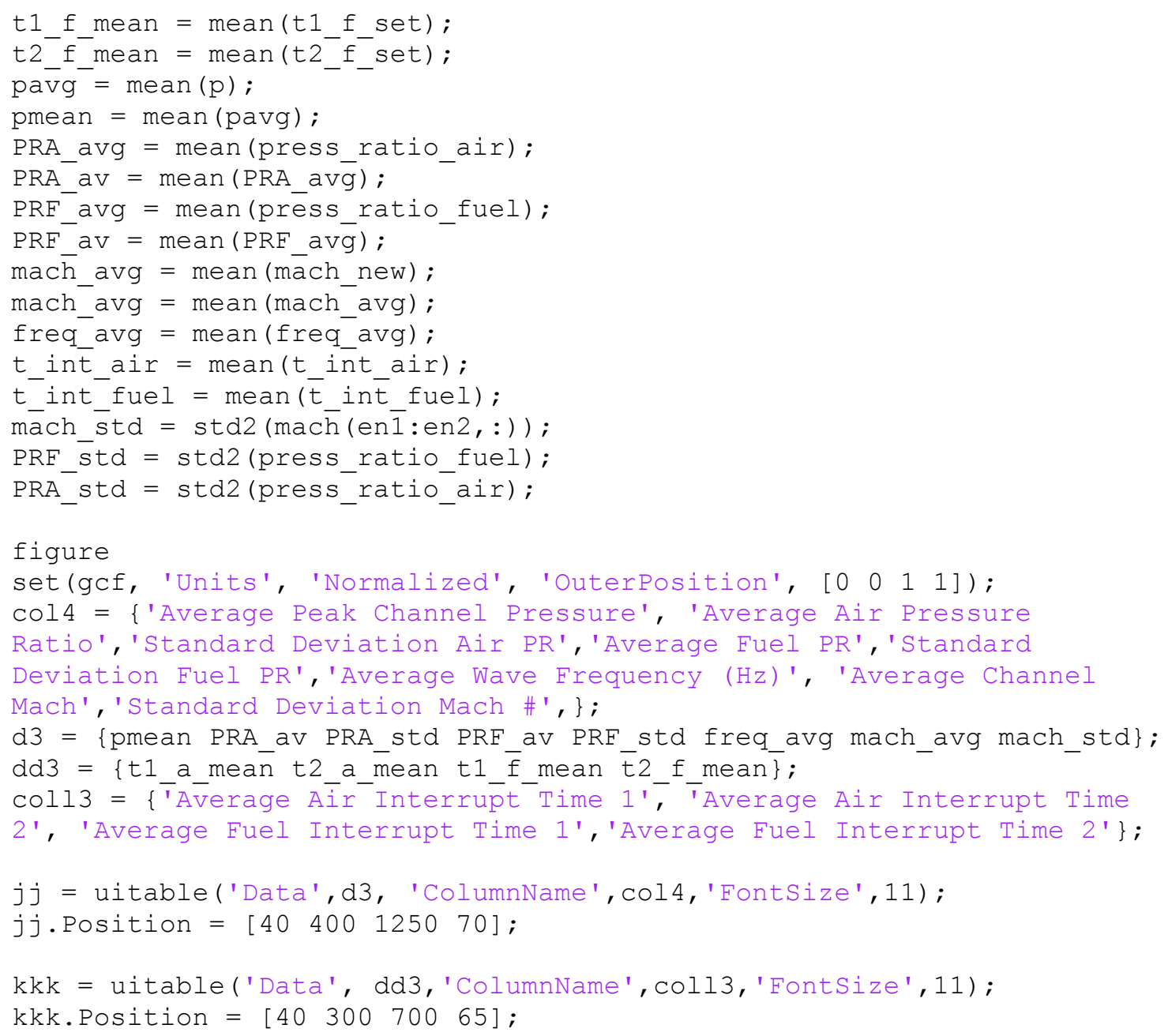




\section{Appendix B: Uncertainty Analysis}

\section{Pressure Measurement Errors}

The dynamic pressure transducers used for this study have an inherent error, which is the maximum uncertainty reported in their literature. The PCB transducers used in the outerbody have an uncertainty of $\pm 1.3 \%$ full scale. They are 200 psig transducers.

$$
\operatorname{err}_{\text {Channel PCBs }}= \pm 2.6 \mathrm{psig}
$$

The static pressure transducers are Omega, and the total error band is $\pm 2.0 \%$ of full scale. The air side sensor is $15 \mathrm{psig}$, and the helium side sensor is 5 psig.

$$
\begin{aligned}
& \operatorname{err}_{\text {Air }}= \pm 0.3 \mathrm{psig} \\
& \operatorname{err}_{\text {Helium }}= \pm 0.1 \mathrm{psig}
\end{aligned}
$$

The dynamic pressure transducers used in the plenums are low range (50 psig), dynamic PCB transducers. They have a sensitivity of $\pm 15 \%$.

$$
\operatorname{err}_{\text {Plenum PCBS }}= \pm 7.5 \mathrm{psig}
$$

\section{Wave Speed Error}

Wave speeds are calculated based on the peaks of the pressure signals, the distance between the transducers, and the recorded time difference.

$$
V_{\text {wave }}=\frac{x_{2}-x_{1}}{t_{2}-t_{1}}
$$

The distance between the transducers was measured with calipers, which had a resolution of 0.0005 inches.

$$
\left(x_{2}-x_{1}\right)=(1.1 \pm 0.0005) "
$$




$$
\operatorname{err}_{\Delta x}= \pm 0.045 \%
$$

From the Nyquist sampling theorem, digitized time samples are only certain as half of their sampling rate:

$$
\left(\frac{n_{1}}{f_{s}} \pm \frac{1}{2 f_{s}}\right)-\left(\frac{n_{2}}{f_{s}} \pm \frac{1}{2 f_{s}}\right)
$$

Depending on the set of measurements, the $\Delta \mathrm{n}$ is different. For the smallest $\Delta \mathrm{n}$ value of 7:

$$
\operatorname{err}_{\Delta t}=14.29 \%
$$

These errors combine for an equivalent error of:

$$
\begin{gathered}
\operatorname{err}_{W S}=\sqrt{0.045^{2}+14.29^{2}} \\
e r r_{W S}=14.29 \%
\end{gathered}
$$

\section{Flow Rate Errors}

A combination of Alicat and Omega flow controllers were used. The accuracy of the Alicat controllers is \pm ( $0.8 \%$ of reading $+0.2 \%$ of full scale $)$. Based on the flow rate sweeps, a range of possible error can be stated. Alicat mass flow controllers of $50 \mathrm{slpm}$ and 500 slpm were used.

$$
\begin{gathered}
\operatorname{err}_{\text {Alicat } 50}= \pm(0.008 * Q+0.1) \text { slpm } \\
\text { Range err }{ }_{\text {Alicat } 50}= \pm(0.8 * Q+0.1) \text { slpm } \\
\operatorname{err}_{\text {Alicat } 500}= \pm(0.008 * Q+1) \text { slpm } \\
\text { Range err } \text { Alicat } 500=1.8-4 \text { slpm }
\end{gathered}
$$


The Omega flow controllers have an accuracy of $\pm 1 \%$ full scale. A 100 and 200 slpm Omega controller are used.

$$
\begin{aligned}
& \text { err }_{\text {Omega } 100}= \pm 1 \text { slpm } \\
& \text { err }_{\text {Omega } 200}= \pm 2 \text { slpm }
\end{aligned}
$$

